\title{
DIFFERENTIALS OF COMPLEX INTERPOLATION PROCESSES FOR KÖTHE FUNCTION SPACES
}

\author{
N. J. KALTON
}

\begin{abstract}
We continue the study of centralizers on Köthe function spaces and the commutator estimates they generate (see [29]). Our main result is that if $X$ is a super-reflexive Köthe function space then for every real centralizer $\Omega$ on $X$ there is a complex interpolation scale of Köthe function spaces through $X$ inducing $\Omega$ as a derivative, up to equivalence and a scalar multiple. Thus, in a loose sense, all real centralizers can be identified with derivatives of complex interpolation processes. We apply our ideas in an appendix to show, for example, that there is a twisted sum of two Hilbert spaces which fails to be a (UMD)-space.
\end{abstract}

\section{INTRODUCTION}

In this paper we develop ideas first suggested by work of Rochberg and Weiss [45] and continued in [29]. Our basic program is to study "twisted sums" of Banach spaces (see $[24,25,26,32,35]$ ) as differentials of interpolation processes, and the corresponding commutator estimates obtained for linear operators. In [29] we gave several applications of these ideas, in particular applying them to obtain results of Davis [17] and Ceretelli [10] on the distributions of functions in the Hardy class $H^{1}$. (See also [27, 30].) Our results in this paper complement and improve the results we obtained in [29]. In this paper we study general Köthe function spaces in place of rearrangement-invariant spaces and use a different approach. The main idea here is to characterize those twisted sums of Köthe function spaces which can be obtained by differentiating a complex interpolation scale of Köthe function spaces.

Let us now give some definitions and an informal discussion of our main results.

If $0 \rightarrow Y \rightarrow Z \rightarrow X \rightarrow 0$ is an exact sequence of (quasi-)Banach spaces (over the field $\mathbf{K}=\mathbf{R}$ or $\mathbf{C}$ ) we refer to $Z$ as a twisted sum of $X$ and $Y$ (or an extension of $X$ by $Y$ ). If $\operatorname{dim} Y=1$, we say that $Z$ is a minimal extension of $X$.

Suppose $X$ is a quasi-Banach space with a dense linear subspace $X_{0}$ and that $Y$ is a quasi-Banach space contained in a linear space $\tilde{Y}$ (which does not necessarily carry any topology). Consider a map $\Omega: X_{0} \rightarrow \tilde{Y}$ satisfying, for

Received by the editors August 14, 1990.

1991 Mathematics Subject Classification. Primary 46M35, 46E30, Secondary 42A50, 46B42, 47B38, 47D25.

This research was supported by NSF grants DMS-8601401 and DMS-8901626. 
suitable $\rho=\rho(\Omega)$,

$$
\begin{gathered}
\Omega(\alpha x)=\alpha \Omega(x), \quad \alpha \in \mathbf{K}, x \in X, \\
\left\|\Delta_{\Omega}\left(x_{1}, x_{2}\right)\right\|_{Y} \leq \rho\left(\left\|x_{1}\right\|_{X}+\left\|x_{2}\right\|_{X}\right), \quad x_{1}, x_{2} \in X_{0},
\end{gathered}
$$

where we introduce the notation

$$
\Delta_{\Omega}\left(x_{1}, \ldots, x_{n}\right)=\sum_{k=1}^{n} \Omega\left(x_{k}\right)-\Omega\left(\sum_{k=1}^{n} x_{k}\right) .
$$

Then we can create a twisted sum $X \oplus_{\Omega} Y$ as the completion of the space $Z_{0}$ of all $(x, y) \in X_{0} \oplus \widetilde{Y}$ such that

$$
\|(x, y)\|_{Z}=\|x\|_{X}+\|y-\Omega(x)\|_{Y}<\infty .
$$

Conversely every twisted sum of $X$ and $Y$ can be described in this way taking $X=X_{0}$ and $Y=\tilde{Y}$ (see $\left.[24,33,44]\right)$.

We refer to [2, 3] for a general discussion of interpolation theory. In [23, 45], Jawerth, Rochberg, and Weiss have shown that twisted sums arise very naturally in interpolation theory, as the 'differential' of an interpolation process. The complex method is treated in [45] (see also some similar ideas in [47]) and real methods are considered in [23]. The results are surveyed in [15]. See also related work in [40]. We would also like to mention the work of Coifman and Semmes $[14,48]$ which seems somewhat related to our ideas; also see the work of Slodkowski [49].

In the discussion that follows we will try to give the flavor of the ideas of the paper, without perhaps being too precise about technical definitions.

Suppose $X_{0}, X_{1}$ is a pair of Banach spaces and $X=X_{\theta}=\left[X_{0}, X_{1}\right]_{\theta}$ is an intermediate space obtained by the complex method of interpolation. Then Rochberg and Weiss associate to the scale $\left[X_{0}, X_{1}\right]$ and $X$ a map $\Omega: X \rightarrow$ $X_{0}+X_{1}$ satisfying (1.1) and (1.2) in such a way that if $T$ is a linear operator bounded on both $X_{0}$ and $X_{1}$ then $T \oplus T$ is also bounded on $X \oplus_{\Omega} X$ (which we will call $d_{\Omega} X$ later in the paper). This implies a commutator relationship

$$
\|[T, \Omega](x)\|_{X} \leq C\|x\|_{X},
$$

where $[T, \Omega]=T \Omega-\Omega T$.

Now consider a Köthe function space $X$; for the sake of definiteness let us consider spaces over [0,1]. In [29], the author defined a map $\Omega: X \rightarrow L_{0}$ to be a (homogeneous) centralizer if it satisfies (1.1) and (1.3) uniformly for all multiplication operators, i.e.,

$$
\|\Omega(u x)-u \Omega(x)\|_{X} \leq \rho\|u\|_{\infty}\|x\|_{X}
$$

for $f \in X, u \in L_{\infty}$ where $\rho(\Omega)$ is a constant independent of $u, f$. It turns out that (1.2) then also holds. $\Omega$ is called symmetric if for some $\rho^{\prime}$

$$
\|\Omega(x \circ \sigma)-\Omega(x) \circ \sigma\|_{X} \leq \rho^{\prime}\|x\|_{X}
$$

for all measure-preserving rearrangements $\sigma$ of $[0,1]$. The work of Jawerth, Rochberg, and Weiss then shows that if $p_{0}<p<p_{1}$ and $T$ is an operator of strong types $\left(p_{0}, p_{0}\right)$ and $\left(p_{1}, p_{1}\right)$ then $(1.3)$ holds for certain symmetric centralizers on $L_{p}$ such as

$$
\Omega(x)=x \log \frac{|x|}{\|x\|_{p}} \text { and } \Omega(x)=x \log r_{x},
$$


where $r_{x}(t)=\mu\{x:|f(s)|>|f(t)|$ or $|f(s)|=|f(t)|$ and $s \leq t\}$. The aim in [29] was to show that the same hypotheses in fact ensure that (1.3) holds for every symmetric centralizer.

In this paper our objective is to show at least for super-reflexive Köthe function spaces $X$ that every centralizer with the additional property that $\Omega(x)$ is a real function whenever $x$ is real ( $\Omega$ is real) can be obtained by differentiating a suitable complex interpolation scale of Köthe function spaces. Precisely, we show that if $X$ is a super-reflexive Köthe function space and $\Omega: X \rightarrow L_{0}$ is a real centralizer on $X$ then there are constants $a>0$ and $B<\infty$ and Köthe function spaces $X_{0}, X_{1}$ so that $X=\left[X_{0}, X_{1}\right]_{1 / 2}$ (isomorphically) and if $\Omega_{0}$ is the induced centralizer on $X$ obtained by differentiation then

$$
\left\|\Omega(x)-a \Omega_{0}(x)\right\|_{X} \leq B\|x\|_{X}
$$

for all $x \in X$. The spaces $X_{0}, X_{1}$ are determined up to isomorphism by $\Omega$ and $a$; if $X$ is rearrangement-invariant and $\Omega$ is symmetric then both $X_{0}$ and $X_{1}$ are rearrangement-invariant. Thus the main interpolation theorem of [29] can be recaptured as a consequence of the Boyd interpolation theorem [5].

The key idea here is to exploit the intimate relationship between centralizers on $X$ and minimal extensions of $L_{1}$. To every Köthe function space we may associate a map $\Phi_{X}: I \rightarrow C$ defined on a dense order ideal in $L_{1}$ and satisfying (1.1) and (1.2). For nonnegative functions $f$

$$
\Phi_{X}(f)=\sup _{\|x\|_{X} \leq 1} \int f \log |x| d \mu .
$$

We then show that to every centralizer $\Omega$ defined on $X$ corresponds a similar densely defined functional $\Phi^{\Omega}$; if $\Omega$ is obtained by differentiating a scale $\left[X_{0}, X_{1}\right]$ then $\Phi^{\Omega}=\Phi_{X_{1}}-\Phi_{X_{0}}$. The main result is then obtained by showing that if $\Phi$ is real for real functions and satisfies (1.1) and (1.2) with $\rho<\log 2$ then it is 'equivalent' to $\Phi_{X}-\Phi_{X^{*}}$ for some suitable Köthe function space $X$.

To illustrate this let us state precisely a finite-dimensional version.

Theorem 1.1. Given $\varepsilon>0$ there exists a constant $B=B(\varepsilon)$ so that if $n \in \mathbf{N}, P$ is the nonnegative cone in $l_{1}^{n}$ and $\Phi: P \rightarrow \mathbf{R}$ is a functional satisfying $\Phi(\alpha x)=$ $\alpha(\Phi(x))$ and

$$
\left|\Delta_{\Phi}\left(x_{1}, x_{2}\right)\right| \leq(1-\varepsilon) \log 2\left(\left\|x_{1}\right\|+\left\|x_{2}\right\|\right),
$$

whenever $x, x_{1}, x_{2} \in P$ and $\alpha>0$, then there exists a lattice norm \|\|$_{X}$ on $\mathbf{R}^{n}$ so that

for $x \in P$.

$$
\left|\Phi(x)-\left(\Phi_{X}(x)-\Phi_{X^{*}}(x)\right)\right| \leq B\|x\|
$$

One could also state this in terms of approximating nearly affine functions on the unit simplex.

We now briefly outline the contents of the paper. In $\S 2$ we set up a formal framework for complex interpolation, including the case of families of spaces studied in $[11,12]$. Our framework is a slight variation on the usual approach as outlined in [3], for example, but seems appropriate to our setting. We introduce the notion of a derivation obtained by differentiating an interpolation scale. In $\S 3$ we study how these ideas adapt to Köthe function spaces. We study in $\S 4$ and $\S 5$ the functional $\Phi_{X}$ which we associate to any Köthe function space. Our 
main approximation theorem (specialized to Theorem 1.1) above is proved in $\S 6$ as Theorem 6.6. Our main result on centralizers is Theorem 7.6. In $\S 8$ we generalize our ideas to Schatten ideals.

Finally in $\S 9$, we apply the methods of the paper to the study of the Riesz projection and its vector-valued analogue. For $f \in L_{1}(\mathbf{T})$ we define $R f$ by $R f \sim \sum_{n>0} \hat{f}(n) e^{i n t}$. We then show that if $X_{0}, X_{1}$ are Köthe function spaces on $\mathbf{T}$ so that $R$ is bounded on $X=X_{\theta_{0}}=\left[X_{0}, X_{1}\right]_{\theta_{0}}$ for some $0<\theta_{0}<1$ where $X$ is super-reflexive then the boundedness of the associated commutator $[R, \Omega]$ on $X$ is equivalent to the boundedness of $R$ on $X_{\theta}$ in a neighborhood of $\theta_{0}$. We show how this relates to and extends several well-known results on $A_{p}$-weights (cf. $\left.[13,41,50]\right)$. In the vector-valued case we can apply the methods to the study of the class of (UMD)-spaces introduced by Burkholder [7, 8]. As an application we show that there is a twisted sum of two Hilbert spaces which fails to be (UMD) thus resolving, negatively, the question of whether the (UMD)-property is a three-space property.

\section{ADMISSIBLE SPACES AND DERIVATIONS}

Let $S$ be a Polish space and let $\mu$ be a $\sigma$-finite Borel measure on $S$. Let $L_{0}=L_{0}(\mu)$ be the space of all measurable complex-valued functions on $S$ endowed with the topology of convergence in measure relative to each Borel set of finite measure. We define an admissible norm to be a map $f \rightarrow\|f\|_{X}$ $\left(L_{0} \rightarrow[0, \infty]\right)$ such that if $X=\left\{x \in L_{0}:\|x\|_{X}<\infty\right\}$ then:

(2.1) $X$ is a vector subspace of $L_{0}$ and \|\|$_{X}$ is a norm on $X$.

(2.2) $B_{X}=\left\{x:\|x\|_{X} \leq 1\right\}$ is closed in $L_{0}$.

(2.3) There exist strictly positive $h, k \in L_{0}$ so that

$$
\|x h\|_{1} \leq\|x\|_{X} \leq\|x k\|_{\infty}
$$

for every $x \in L_{0}$.

The corresponding space $X$ is then easily proved to be a Banach space which is continuously embedded in $L_{0}$. We refer to $X$ as an admissible space. If $X$ is an admissible space then a map $\Omega: X \rightarrow L_{0}$ will be called a derivation if (2.4)-(2.6) hold, where

(2.4) $\Omega$ is homogeneous, i.e., $\Omega(\alpha x)=\alpha \Omega(x), \alpha \in \mathbf{C}, x \in X$.

(2.5) $\Omega$ is quasi-additive, i.e., $\left\|\Delta_{\Omega}\left(x_{1}, x_{2}\right)\right\|_{X} \leq \rho\left(\left\|x_{1}\right\|_{X}+\left\|x_{2}\right\|_{X}\right), x_{1}, x_{2} \in$ $X$, where $\rho$ is a constant independent of $x_{1}, x_{2}$. We refer to the least such constant as $\rho(\Omega)$.

(2.6) The set $\Omega\left(B_{X}\right)$ is bounded in $L_{0}$.

For any derivation we may introduce the derived space $d_{\Omega} X$ as the subset of $L_{0} \times L_{0}$ of all pairs $(x, y)$ such that

$$
\|(x, y)\|_{X, \Omega}=\|x\|_{X}+\|y-\Omega(x)\|_{X}<\infty .
$$

Lemma 2.1. \|\|$_{X, \Omega}$ is a quasinorm on $d_{\Omega} X$ and $d_{\Omega} X$ is a quasi-Banach space which is continuously embedded in $L_{0} \times L_{0}$. If $X$ is a $B$-convex Banach space, or $X^{*}$ is isomorphic to a subspace of an $L_{1}$-space then $d_{\Omega} X$ is a Banach space, and there is a constant $C$ depending only on $X$ so that for any $x_{1}, \ldots, x_{n} \in X$,

$$
\left\|\Delta_{\Omega}\left(x_{1}, \ldots, x_{n}\right)\right\|_{X} \leq C \sum_{k=1}^{n}\left\|x_{k}\right\|_{X} .
$$


Proof. It is easy to check that if $a, b \in d_{\Omega} X$ then

$$
\|a+b\|_{X, \Omega} \leq(1+\rho)\left(\|a\|_{X, \Omega}+\|b\|_{X, \Omega}\right) .
$$

Clearly, if $a_{n}=\left(x_{n}, y_{n}\right)$ and $\left\|a_{n}\right\|_{X, \Omega} \rightarrow 0$ then $\left\|x_{n}\right\|_{X} \rightarrow 0$ and so, by using (2.4) and (2.6), $\Omega\left(x_{n}\right) \rightarrow 0$ in $L_{0}$. From this it follows easily that $y_{n} \rightarrow 0$ in $L_{0}$. Clearly the subspace $\{(0, y): y \in X\}$ is a closed subspace $F$ of $d_{\Omega} X$ such that $F$ is isometric to $X$ and $d_{\Omega} X / F$ is also isometric to $X$. This is easily seen to imply completeness of $d_{\Omega} X$ (cf. [24]).

For the second half of the lemma, we quote the results of [24] and [34] that any Banach space $X$ satisfying either criterion is a $\mathscr{K}$-space, i.e., every twisted sum with a Banach is locally convex. Then if we consider any sequence $\Omega_{n}$ of derivations with $\rho\left(\Omega_{n}\right) \leq 1$ we can construct a derivation on either $l_{2}(X)$ or $l_{\infty}(X)$ according to a case given by $\Omega\left(\left(x_{n}\right)\right)=\left(\Omega_{n}\left(x_{n}\right)\right)$. (We regard both spaces as admissible spaces for a suitable measure space.) Then $\rho(\Omega) \leq 1$. Thus the derived space is locally convex from which it easily follows that there is a constant $C$ independent of $m$, so that

$$
\left\|\Delta_{\Omega_{m}}\left(x_{1}, \ldots, x_{n}\right)\right\|_{X} \leq C \sum_{k=1}^{n}\left\|x_{k}\right\|
$$

for all $x_{1}, \ldots, x_{n}$. The lemma then follows.

We remark that two derivations $\Omega_{1}$ and $\Omega_{2}$ give rise to equivalent derived spaces if for some constant $B$ we have $\left\|\Omega_{1}(x)-\Omega_{2}(x)\right\|_{X} \leq B\|x\|_{X}$. In this case we term $\Omega_{1}, \Omega_{2}$, equivalent.

We denote by $D$ the open unit disk in the complex plane; its boundary, the unit circle, is denoted by $\mathbf{T}$ and $\lambda$ will denote the Haar measure $(2 \pi)^{-1} d \theta$ on T. Now consider a family $\mathscr{X}=\left\{X_{w}\right\}$ of admissible spaces indexed by $w \in \mathbf{T}$; we write $\|x\|_{w}=\|x\|_{X_{w}}$. We say that $\mathscr{X}$ is an admissible family if:

(2.7) The map $(w, x) \rightarrow\|x\|_{w}\left(\mathbf{T} \times L_{0} \rightarrow[0, \infty]\right)$ is a Borel map.

(2.8) There exist strictly positive $h, k \in L_{0}$ so that for all $x \in L_{0}$ we have $\lambda$-a.e. on $\mathbf{T}$,

$$
\|x h\|_{1} \leq\|x\|_{w} \leq\|x k\|_{\infty} .
$$

We may extend this definition to the case when $X_{w}$ is defined only on a Borel subset of $\lambda$-measure one by simply setting $\|x\|_{w}=\|x h\|_{1}$ otherwise.

Let $\mathscr{N}^{+}$be the collection of functions $F: D \rightarrow L_{0}$ which can be written in the form $F(z)(s)=F(z, s)$ where $\mu$-almost everywhere we have $F_{s} \in N^{+}$ where $F_{s}(z)=F(z, s)$ and $N^{+}$denotes the Smirnov class (cf. [18]). If $F \in$ $\mathscr{N}^{+}$it may easily be shown by an application of Fubini's theorem that relative to the $L_{0}$-topology $F$ has radial limits almost everywhere on $\mathbf{T}$. Thus we can define $F\left(e^{i \theta}\right)=\lim _{r \rightarrow 1} F\left(r e^{i \theta}\right)$ almost everywhere.

If $\mathscr{X}$ is an admissible family then we define for $F \in \mathscr{N}^{+}$

$$
\|F\| \mathscr{X}=\operatorname{ess} \sup \left\|F\left(e^{i \theta}\right)\right\|_{e^{i \theta}} .
$$

Then for $z \in D$ we define

$$
\|x\|_{z}=\inf \left\{\|F\|_{\mathscr{L}}: F(z)=x\right\}
$$

and let $X_{z}=\left\{x:\|x\|_{z}<\infty\right\}$. 
Lemma 2.2. Let $\mathscr{X}$ be an admissible family and let $Y$ be a separable admissible space so that $\|x\|_{Y} \leq\|x\|_{w}$ for all $w \in \mathbf{T}$. Then, if $\|F\|_{\mathscr{L}}<\infty$, we have $\|F(z)\|_{Y} \leq\|F\|_{\mathscr{Q}}$ for all $z \in D$. Furthermore $F\left(r e^{i \theta}\right) \rightarrow F\left(e^{i \theta}\right)$ a.e. in $Y$.

For any $z \in D, x \in L_{0}$ we have $\|x\|_{Y} \leq\|x\|_{z}$.

Proof. We may assume the existence of a strictly positive $h$ satisfying (2.3) for $Y$ and (2.8). Then

$$
\begin{aligned}
\int_{-\pi}^{\pi} \int_{S}\left|F\left(e^{i \theta}, s\right)\right| h(s) d \mu(s) \frac{d \theta}{2 \pi} & \leq \int_{S} \int_{-\pi}^{\pi}\left|F\left(e^{i \theta}, s\right)\right| h(s) \frac{d \theta}{2 \pi} d \mu(s) \\
& =\int_{-\pi}^{\pi}\left\|F\left(e^{i \theta}\right)\right\|_{L_{1}(h d \mu)} \frac{d \theta}{2 \pi} \\
& \leq\|F\|_{\mathscr{D}} .
\end{aligned}
$$

Hence

$$
\int_{-\pi}^{\pi}\left\|F\left(r e^{i \theta}\right)\right\|_{L_{1}(h \boldsymbol{d})} \frac{d \theta}{2 \pi} \leq\|F\|_{\mathscr{Z}}
$$

for $0<r<1$ so that $F \in H_{1}\left(L_{1}(h d \mu)\right)$. Since $L_{1}$ has the analytic RadonNikodym Property [6], this implies that $F\left(r e^{i \theta}\right) \rightarrow F\left(e^{i \theta}\right)$ a.e. in $L_{1}(h d \mu)$ and, further, that

$$
F\left(r e^{i \theta}\right)=\int_{-\pi}^{\pi} P(r, \theta-t) F\left(e^{i t}\right) \frac{d t}{2 \pi}
$$

as a Bochner integral in $L_{1}(h d \mu)$, where $P$ is the Poisson kernel. Since $Y$ is separable, $F\left(e^{i t}\right)$ is also measurable in $Y$, and, since it is also bounded in $Y$, the same equation holds as a Bochner integral in $Y$. Hence for $z \in D$ we have $\|F(z)\|_{Y} \leq\|F\|_{\mathscr{L}}$. It is also easy to verify that $F\left(r e^{i \theta}\right) \rightarrow F\left(e^{i \theta}\right)$ in $Y$ almost everywhere.

It now follows that $\|x\|_{z} \geq\|x\|_{Y}$ for any $x \in L_{0}$.

Lemma 2.3. Suppose $F_{n} \in \mathscr{N}^{+}$and $\left\|F_{n}\right\|_{\mathscr{X}} \leq 1$. Then there is a sequence $G_{n}$ of convex combinations of $\left\{F_{k}: k \geq n\right\}$ and $a G \in \mathscr{N}^{+}$so that $\|G\|_{\mathscr{L}} \leq 1$ and

$$
\begin{gathered}
\lim _{n \rightarrow \infty} G_{n}^{(k)}(z)=G^{(k)}(z), \quad z \in D, k=0,1,2, \ldots, \\
\lim _{n \rightarrow \infty} G_{n}\left(e^{i \theta}\right)=G\left(e^{i \theta}\right) \quad \lambda \text {-a.e. },
\end{gathered}
$$

where both limits are computed in $L_{0}$.

Proof. Since

$$
\int_{-\pi}^{\pi} \int_{S}\left|F_{n}\left(e^{i \theta}, s\right)\right| h(s) d \mu(s) \frac{d \theta}{2 \pi} \leq 1,
$$

we can apply Komlos's theorem [36] to extract a sequence $G_{n}$ of convex combinations as specified so that we have both that $G_{n}\left(e^{i \theta}, s\right)$ converges $\mu \times \lambda$-a.e. and $\int_{-\pi}^{\pi}\left|G_{n}\left(e^{i \theta}, s\right)\right| d \theta / 2 \pi$ is bounded $\mu$-a.e. (The sequence of convex combinations $\sum_{k=n}^{N(n)} a_{k}^{n} F_{k}$ is chosen so that $\mu$-a.e.

$$
\int_{-\pi}^{\pi} \sum_{k=n}^{N(n)} a_{k}^{n}\left|F_{k}\left(e^{i \theta}, s\right)\right| \frac{d \theta}{2 \pi}
$$

converges.) 
If we write $G_{n}(z, s)=\sum_{j=0}^{\infty} g_{n, j}(s) z^{j}$ then we have $G_{n, s} \in N^{+} \mu$-a.e. Let $S_{0}$ be the set of $s \in S$ such that we have, simultaneously, $G_{n, s} \in N^{+}, G_{n}\left(e^{i \theta}, s\right)$ converges $\lambda$-a.e. and $\int_{-\pi}^{\pi}\left|G_{n}\left(e^{i \theta}, s\right)\right| d \theta / 2 \pi$ is bounded. Then $\mu\left(S \backslash S_{0}\right)=0$.

For $s \in S_{0}$ the sequence $G_{n, s}$ is uniformly bounded in $L_{1}(\mathbf{T}) \cap N^{+}=$ $H_{1}(\mathbf{T})(\mathbf{T})$ and converges a.e. Hence it converges in $L_{p}(\mathbf{T})$ for every $p<1$ to a function $G_{s} \in H_{p}(\mathbf{T})$. Further if $z \in D, k \geq 0, \lim _{n \rightarrow \infty} G_{n, s}^{(k)}(z)=G_{s}^{(k)}(z)$ while on $\mathbf{T}, \lim _{n \rightarrow \infty} G_{n, s}\left(e^{i \theta}\right)=G_{s}\left(e^{i \theta}\right)$ a.e. Thus for each $j, g_{n, j}$ converges in $L_{0}$ (in fact a.e.) to some $g_{j} \in L_{0}$ and, if $G(z)=\sum g_{j} z^{j}$, then for a.e. $s \in S G(z, s)=G_{s}(z)$. Hence $G \in \mathscr{N}^{+}$and $\|G\|_{\mathscr{Z}} \leq 1$.

Proposition 2.4. Each space $X_{z}$ is admissible and if $x \in X_{z}$ there is an extremal $F \in \mathscr{N}^{+}$such that $F(z)=x$ and $\|F\|_{\mathscr{X}}=\|x\|_{z}$.

Proof. Conditions (2.1) and (2.3) are elementary. We prove only (2.2), and simultaneously prove the existence of an extremal. To do this we need only consider a sequence $x_{n}$ with $\left\|x_{n}\right\|_{z}<1$ so that $x_{n} \rightarrow x$ a.e. and prove that this implies the existence of $F \in \mathscr{N}^{+}$with $F(z)=x$ and $\|F\|_{\mathscr{X}} \leq 1$. However, there exist $F_{n} \in \mathscr{N}^{+}$with $\left\|F_{n}\right\|_{\mathscr{Z}} \leq 1$ and $F_{n}(z)=x_{n}$ and so the conclusion follows easily from Lemma 2.3 .

These considerations can now be lifted to any open subset $U$ of $\mathbf{C}$ conformally equivalent to $D$. We define an admissible family of spaces $\mathscr{X}=$ $\left(X_{w}: w \in \partial U\right)$ to be a family of admissible spaces so that

(2.11) The map $(w, x) \rightarrow\|x\|_{w}$ is Borel on $\partial U \times L_{0}$.

(2.12) There exist strictly positive $h, k \in L_{0}$ so that for $x \in L_{0}$ we have for every $w \in \partial U,\|x h\|_{1} \leq\|x\|_{w} \leq\|x k\|_{\infty}$.

Let $\varphi: D \rightarrow U$ be any conformal equivalence. Then $\varphi \in H_{p}$ for any $p<\frac{1}{2}$ [18] and so has radial limits $\varphi\left(e^{i \theta}\right) \in \partial U$ a.e. The family $\varphi(\mathscr{X})=\left(X_{\varphi\left(e^{i \theta}\right)}\right)$ is then admissible for $D$. Define $\mathcal{N}^{+}(U)$ to be the space of all functions $F: U \rightarrow L_{0}$ for which $F \circ \varphi \in \mathcal{N}^{+}$and set $\|F\|_{\mathscr{X}}=\|F \circ \varphi\|_{\varphi(\mathscr{X})}$. It is easy to show that these definitions are independent of $\varphi$. For $z \in U$ we define

$$
\|x\|_{z}=\inf \left\{\|F\|_{\mathscr{L}}: F(z)=x\right\}
$$

and set $X_{z}=\left\{x:\|x\|_{z}<\infty\right\}$. We then automatically obtain

Proposition 2.5. Each $X_{z}$ is admissible and if $x \in X_{z}$ then there is an extremal $F \in \mathscr{N}^{+}(U)$ with $F(z)=x$ and $\|F\|_{\mathscr{X}}=\|x\|_{z}$.

In particular if $X_{0}, X_{1}$ is a pair of admissible spaces we let $U=\mathscr{S}$ be the strip $\{z: 0<\Re z<1\}$ and define $X_{k+i t}=X_{k}$ for $k=0,1$ and $t \in \mathbf{R}$. Then for $0<\theta<1$ we obtain the complex interpolation space $X_{\theta}=\left[X_{0}, X_{1}\right]_{\theta}$.

Now let $U$ be any open subset of $\mathbf{C}$ which is conformally equivalent to $D$ and let $\mathscr{X}$ be an admissible family. For $z \in U$ and $x, y \in L_{0}$ we define

$$
\|(x, y)\|_{d X_{z}}=\inf \left\{\|F\|_{\mathscr{X}}: F(z)=x, F^{\prime}(z)=y\right\} .
$$

For each $x \in X_{z}$ we may pick an extremal $F_{x}$ by Proposition 2.5 with $\left\|F_{x}\right\|_{\mathscr{X}}=$ $\|x\|_{z}$ and $F_{x}(z)=x$. We may further suppose that $F_{\alpha x}=\alpha F_{x}$ for $\alpha \in \mathbf{C}$. Then we define $\Omega=\Omega_{\mathscr{X}, z}$ by $\Omega(x)=F_{x}^{\prime}(z)$. 
Theorem 2.6. $\Omega$ is a derivation on $X_{z}$ and \|\|$_{d X_{z}}$ is equivalent to \|\|$_{X_{z}, \Omega}$.

Remark. Theorem 2.6 is essentially due to Rochberg and Weiss [45]. If each $X_{w}$ is strictly convex, then, as they observe, the extremal $F_{x}$ is unique and hence $\Omega$ is uniquely determined. See also Corollary 4.9 below for more discussion of the uniqueness question.

Proof. Property (2.4) of derivations is built into our construction. Let $\varphi: U \rightarrow$ $D$ be a conformal equivalence, with $\varphi(z)=0$, and let $\left|\varphi^{\prime}(z)\right|=\delta$. Then

$$
\begin{aligned}
\|\Omega(x)\|_{L_{1}(h d \mu)} & =\left\|F_{x}^{\prime}(z)\right\|_{L_{1}(h d \mu)} \\
& \leq \delta \operatorname{ess} \sup \left\|F_{x}\left(e^{i \theta}\right)\right\|_{L_{1}(h d \mu)} \\
& \leq \delta\|x\|_{X_{z}},
\end{aligned}
$$

so that property $(2.6)$ holds. The remaining statements are essentially contained in [45]. It is readily verified that $\|(0, x)\|_{d X_{z}}=\delta\|x\|_{X_{z}}$. It then follows from the triangle law for the norm on $d X_{z}$ that $\left\|\Delta_{\Omega}(x, y)\right\|_{X_{z}} \leq 2 \delta\left(\|x\|_{X_{z}}+\|y\|_{X_{z}}\right)$ so that $(2.5)$ hold with $\rho=2 \delta$. It can also be checked that

$$
\min \left(1, \delta^{-1}\right)\|(x, y)\|_{d X_{z}} \leq\|(x, y)\|_{X_{z}, \Omega} \leq(1+2 \delta)\|(x, y)\|_{d X_{z}} .
$$

Finally, let us observe, in our setting, the standard commutator interpolation theorem for linear operators.

Theorem 2.7. Suppose $\mathscr{X}$ is admissible family for $U$, where $U$ is conformally equivalent to $D$. Let $Y$ be a separable admissible space such that \|\|$_{Y} \leq\|\|_{w}$ for $w \in \partial U$. Let $T: Y \rightarrow L_{0}$ be a continuous linear map such that for some $M<\infty$ and every $w \in U, x \in X_{w}\|T x\|_{w} \leq M\|x\|_{w}$.

Then for $z \in U$ we have $T\left(X_{z}\right) \subset X_{z}$ and $\|T\|_{X_{z} \rightarrow X_{z}} \leq M$. Further there is a constant $C=C(z)$ so that for every $x \in X_{z}$

$$
\|[T, \Omega] x\|_{X_{z}} \leq C M\|x\|_{z},
$$

where $\Omega=\Omega_{\mathscr{X}, z}$ and $[T, \Omega]=T \Omega-\Omega T$.

Proof. From Nikishin's theorem [39,42] for any fixed $0<p<1$ there exists a strictly positive $h_{0} \in L_{0}$ so that for any $y \in Y$ we have $\left\|h_{0} T y\right\|_{p} \leq\|y\|_{Y}$. Let $\varphi: D \rightarrow U$ be a conformal equivalence with $\varphi(0)=z$.

Suppose $\|x\|_{X_{z}}=1$. Then $F=F_{x} \circ \varphi$ is a bounded analytic function with values in $Y$ and has radial limits a.e. in $Y$ by Lemma 2.2. Let $F_{x} \circ \varphi(\zeta)=$ $\sum_{k=0}^{\infty} u_{k} \zeta^{k}$. Then let $G(\zeta)=\sum_{k=0}^{\infty} T u_{k} \zeta^{k}$. Then $\left\|h_{0} G(\zeta)\right\|_{p} \leq 1$ for all $\zeta \in D$. It follows easily that $G(\zeta, s) \in H_{p}$ for almost every $s \in S$ so that $G \in \mathcal{N}^{+}$. On $\mathbf{T}$ we have, almost everywhere that $G\left(e^{i \theta}\right)=\lim _{r \rightarrow 1} T F\left(r e^{i \theta}\right)$ in $L_{0}$ so that, again by Lemma $2.2 G\left(e^{i \theta}\right)=T F\left(e^{i \theta}\right)$. Hence $\left\|G \circ \varphi^{-1}\right\|_{\mathscr{X}} \leq M$. Thus $\|T x\|_{z} \leq M\|x\|_{z}$.

If we let $G_{0}=G \circ \varphi^{-1}$ we obtain $\left\|\left(T x, G_{0}^{\prime}(z)\right)\right\|_{d X_{z}} \leq M$ or

$$
\left\|\left(T x, T F_{x}^{\prime}(z)\right)\right\|_{d X_{z}} \leq M \text {. }
$$

This implies that $\|[T, \Omega] x\|_{X_{z}} \leq C(z) M$ as required.

\section{FUNCTION SPACES}

We define a Köthe function space $X$ on $S$ to be an admissible space for which \|\|$_{X}$ is a lattice norm i.e., $\|x\|_{X} \leq\|y\|_{X}$ whenever $|x| \leq|y|$. Since, by 
assumption $B_{X}$ is closed in $L_{0}$ it is not difficult to see that we restrict attention to those function spaces which are maximal in the sense of LindenstraussTzafriri [37, p. 118]. We define the dual $X^{*}$ to be the function space defined by

$$
\|y\|_{X^{*}}=\sup _{x \in B_{X}} \int_{S}|x y| d \mu .
$$

Our assumptions force $X^{*}$ to be a norming subspace of the full dual and $X^{*}$ is a function space.

If $X$ is a function space, then a (homogeneous) centralizer is a map $\Omega: X \rightarrow$ $L_{0}$ satisfying (2.4), (2.6), and

$$
\|\Omega(u x)-u \Omega(x)\|_{X} \leq \rho\|x\|_{X}
$$

whenever $\|u\|_{\infty} \leq 1$ and $x \in X$. Here $\rho$ is a constant independent of $x$ and we define $\rho(\Omega)$ to be the least such constant. It is proved in [29] Lemma 4.2 , that a centralizer is automatically a derivation. Conversely we have from Theorem 2.7:

Proposition 3.1. Suppose $U$ is conformally equivalent to $D$ and $\mathscr{X}$ is an admissible family of Köthe function spaces on $S$. Then for $z \in U, X_{z}$ is a Köthe function space and the corresponding derivation $\Omega$ is a centralizer on $X_{z}$.

If we interpolate between two Köthe function spaces $X_{0}$ and $X_{1}$ then it is well known (due to Calderon [9]) that $X_{\theta}$ is given by $X_{\theta}=X_{0}^{1-\theta} X_{1}^{\theta}$ i.e.,

$$
\|x\|_{X_{\theta}}=\inf \left\{\max \left(\|u\|_{X_{0}},\|v\|_{X_{1}}\right):|x|=u^{1-\theta} v^{\theta}, u, v \geq 0\right\} .
$$

Furthermore the extremal $F_{x}$ is given by

$$
F_{x}(z)=(\operatorname{sgn} x) u^{1-z} v^{z},
$$

where $u v$ is the optimal factorization; the fact that an optimal factorization exists follows easily from the existence of an extremal. Thus the corresponding derivation is given by

$$
\Omega(x)=F_{x}^{\prime}(\theta)=x(\log v-\log u) .
$$

Let us note at this point, that there is some ambiguity in this definition since there may not be a unique optimal factorization. At such $x$ we must make a selection, and thus $\Omega$ is only fixed up to equivalence. Fortunately, this does not present a significant complication and many situations (such as when $X_{0}$ and $X_{1}$ have strictly convex norms) uniqueness is guaranteed.

We remark that the identity $X^{1 / 2} X^{* 1 / 2}=L_{2}$ is an important result of Lozanovskii [38], (see also [20]) which asserts in our setting that if $f \in L_{1}$ then $f$ has a unique factorization $f=x y$ where $\|x\|_{X}=1,\|y\|_{X^{*}}=\|f\|_{1}, x \geq 0$, and $\operatorname{supp} x=\operatorname{supp} y=\operatorname{supp} f$. We refer to this factorization as the Lozanovskii factorization of $f$ for $X$. Note here the connection with the idea of the duality map on $X$, whose relationship to differential estimates was observed in [15].

Let us now extend these ideas to the case of interpolation of families. We consider only the case $U=D$. To avoid certain measurability difficulties, we say that an admissible family $\mathscr{X}$ is strongly admissible if it is admissible and there is a countable dimensional subspace $V$ of $L_{0}$ so that $V \cap B_{X_{w}}$ is $L_{0^{-}}$ dense in $B_{X_{w}}$ for almost every $w \in \mathbf{T}$. It is easy to see that this holds in most reasonable situations. 
We introduce a Köthe function space $\mathscr{E}$ on $S \times \mathbf{T}$ by setting

$$
\|\phi\|_{\mathscr{E}}=\operatorname{ess} \sup \left\|\phi\left(\cdot, e^{i \theta}\right)\right\|_{X_{i \theta}} .
$$

Lemma 3.2. Suppose $\mathscr{X}$ is a strongly admissible family of Köthe function spaces. Then $\mathscr{X}^{*}=\left\{X_{w}^{*}, w \in \mathbf{T}\right\}$ is an admissible family and

$$
\|\psi\|_{\mathscr{E} *}=\int_{-\pi}^{\pi}\left\|\psi\left(\cdot, e^{i t}\right)\right\|_{X_{e^{*}}} \frac{d t}{2 \pi} .
$$

We omit the proof of this lemma which is routine. The following theorem is essentially due to Hernandez [22].

Theorem 3.3. Let $\mathscr{Z}$ be a strongly admissible family of Köthe function spaces for $D$. For $z \in D, z=r e^{i \theta}$, we have $\|x\|_{z}=\inf \|\phi\|_{\mathscr{E}}$ where the infimum is taken over all $\phi \in \mathscr{E}$ such that $\phi \geq 0$ and

$$
|x(s)|=\exp \left(\int_{-\pi}^{\pi} P(r, \theta-t) \log \phi\left(s, e^{i t}\right) \frac{d t}{2 \pi}\right) .
$$

Furthermore there is an extremal choice of $\phi$ for which the corresponding centralizer $\Omega=\Omega_{\mathscr{X}, z}$ is given by

$$
\Omega(x)(s)=x(s)\left(\int_{-\pi}^{\pi} \frac{e^{i t}}{\left(e^{i t}-z\right)^{2}} \log \phi\left(s, e^{i t}\right) \frac{d t}{\pi}\right) .
$$

Proof. We first remark that if $\phi \in \mathscr{E}_{+}$, then

$$
\int_{S} \int_{-\pi}^{\pi} \phi\left(s, e^{i t}\right) h(s) \frac{d t}{2 \pi} d \mu(s)<\infty
$$

so that for a.e. $s \log _{+} \phi\left(s, e^{i t}\right)$ is integrable. Thus the integrals in the statement of the theorem are unambiguously defined, but may be $-\infty$.

For $\phi \in \mathscr{E}_{+}$define

$$
F(z, s)=\exp \left(\int_{-\pi}^{\pi} \frac{e^{i t}+z}{e^{i t}-z} \log \phi\left(s, e^{i t}\right) \frac{d t}{2 \pi}\right),
$$

where $F(z, s)=0$ at all $s$ such that $\log \phi$ is not integrable. Then $F \in \mathcal{N}^{+}$, $\left|F\left(e^{i t}, s\right)\right| \leq \phi\left(s, e^{i t}\right)$ a.e. and

$$
\left|F\left(r e^{i \theta, s}\right)\right|=\exp \left(\int_{-\pi}^{\pi} P(r, \theta-t) \log \phi\left(s, e^{i t}\right) \frac{d t}{2 \pi}\right) .
$$

We conclude that $\|x\|_{z} \leq \inf \|\phi\|_{\mathscr{E}}$ in the statement of the theorem. However conversely there exists an extremal $F_{x} \in \mathscr{N}^{+}$and

$$
|x(s)|=|F(z, s)| \leq \exp \left(\int_{-\pi}^{\pi} P(r, \theta-t) \log \left|F\left(e^{i t}, s\right)\right| \frac{d t}{2 \pi}\right) .
$$

Now there exists $u$ with $0 \leq u \leq 1$ so that if $\phi\left(s, e^{i t}\right)=u(s)\left|F\left(e^{i t}, s\right)\right|$ then $\|\phi\|_{\mathscr{E}}=\|x\|_{z}$ and

$$
|x(s)|=\exp \left(\int_{-\pi}^{\pi} P(r, \theta-t) \log \left|\phi\left(s, e^{i t}\right)\right| \frac{d t}{2 \pi}\right) .
$$

This choice of $\phi$ then is optimal and further gives rise to an extremal $F \in \mathscr{N}^{+}$. The last part of the theorem now follows by evaluating its derivative. 
We conclude this section by introducing some further definitions which will be useful in the next section. We will need to consider certain quasi-Banach function spaces. Let us say that a $g$-convex quasinorm on $L_{0}$ is a functional $x \rightarrow\|x\|_{X} \quad\left(L_{0} \rightarrow[0, \infty]\right)$ such that if $X=\left\{x:\|x\|_{X}<\infty\right\}$, then (2.2) holds, $B_{X}$ contains some strictly positive element (this is equivalent to half of (2.3)) and

(3.4) $\|\alpha x\|_{X}=|\alpha|\|x\|_{X}, \alpha \in \mathbf{C}, x \in X$.

(3.5) For every $x, u, v \in L_{0}$ and $0 \leq \theta \leq 1$ we have that if $|x| \leq|u|^{\theta}|v|^{1-\theta}$ then $\|x\|_{X} \leq\|u\|_{X}^{\theta}\|v\|_{X}^{1-\theta}$.

(3.6) Suppose for $0<\tau<\infty$ we define $\eta_{X}(\tau)$ to be the least $\eta$ such that if $x, y \in X$ have disjoint supports and $\|x\|_{X} \leq 1,\|y\|_{X} \leq \tau$ then $\|x+y\|_{X} \leq 1+\eta_{X}(\tau)$. Then $\eta(\tau)<\infty$ for all $\tau$ and $\lim _{\tau \rightarrow 0} \eta_{X}(\tau)=0$.

Here condition (3.6) forces \|\|$_{X}$ to be a quasinorm on $X$ and hence $X$ becomes a quasi-Banach lattice using (3.5). In addition (3.6) imposes a mild continuity property on the quasinorm, while (3.5) (geometrical convexity) implies that $X$ is A-convex in the sense of [28] (see Theorem 4.4 of [28]). In fact if $X$ is A-convex it is automatically renormable to be $p$-convex for some $p>0$. We recall that $X$ is $p$-convex if for every $x_{1}, \ldots, x_{n} \in X$ we have

$$
\left\|\left(\sum_{i=1}^{n}\left|x_{i}\right|^{q}\right)^{1 / q}\right\|_{X} \geq\left(\sum_{i=1}^{n}\left\|x_{i}\right\|_{X}^{q}\right)^{1 / q} .
$$

We remark that if $X$ is $p$-convex then $X^{p}$ is a Köthe function space where $X^{p}$ is defined by $\|x\|_{X^{p}}=\left\||x|^{1 / p}\right\|_{X}^{p}$.

\section{INDICATORS OF KÖTHE FUNCTION SPACES}

We shall say that a subset $\mathscr{I}$ of the positive cone $L_{1}^{+}$of $L_{1}(\mu)$ is a semiideal if $\mathscr{I}$ is a cone and if $0 \leq f \leq g \in \mathscr{I}$ implies $f \in \mathscr{I}$. I is called a strict semi-ideal if it contains a strictly positive member. Let $X$ be a $g$-convex function space on $S$. We define $\mathscr{I}_{X}$ to be the semi-ideal of all $f \in L_{1}^{+}$such that

$$
\sup _{x \in B_{X}} \int_{S} f \log _{+}|x| d \mu<\infty
$$

and

$$
\exists x \in B_{X}, \quad \int_{S} f|\log | x \| d \mu<\infty .
$$

For $f \in \mathscr{I}_{X}$ we define the indicator of $X, \Phi_{X}$, by

$$
\Phi_{X}(f)=\sup _{x \in B_{X}} \int_{S} f \log |x| d \mu
$$

and then $-\infty<\Phi_{X}(f)<\infty$. It is appropriate to note that this idea is suggested by Gillespie's proof of the Lozanovskii theorem [20].

Let us start by identifying $\Phi_{X}$ for some simple spaces. We shall henceforward write $\|f\|$ without subscript for $\|f\|_{1}$. We say that $f \in L \log L$ if $f(1+|(\log |f|)|) \in L_{1}$. 
Lemma 4.1. Suppose $X=L_{1}$. Then $\mathscr{I}_{X}=(L \log L)_{+}$and

$$
\Phi_{X}(f)=\int f \log \frac{f}{\|f\|} d \mu .
$$

Proof. If $f \in(L \log L)_{+}$and $\|f\|=1$ then for $\|x\| \leq 1$,

$$
\begin{aligned}
\int f \log _{+}|x| d \mu & =\int_{|x|>1} f \log |x| d \mu \\
& =\int_{|x|>1} f \log f d \mu+\int_{|x|>1} f \log \frac{|x|}{f} d \mu \\
& \leq \int_{|x|>1} f \log |f| d \mu+\frac{1}{e} \int_{|x|>1}|x| d \mu \\
& \leq \int f|\log f| d \mu+e^{-1} .
\end{aligned}
$$

Since $f \log f \in L_{1},(4.1)$ and (4.2) hold so that $(L \log L)_{+} \subset \mathscr{J}_{X}$. Conversely if $f \in \mathscr{F}_{X}$ then for some $x \in B_{X} f \log |x|$ is integrable and by the Geometric Mean Inequality,

$$
\int f \log \frac{|x|}{f} d \mu \leq \log \int|x| d \mu \leq 0,
$$

so that

$$
\int f \log |x| d \mu \leq \int f \log f d \mu .
$$

Since $f \log _{+} f$ is integrable we obtain both that $\mathscr{I}_{X}=(L \log L)_{+}$and that $\Phi_{X}(f)=\int f \log f d \mu$ and this in turn implies the lemma for general $f$ by homogeneity of $\boldsymbol{\Phi}_{X}$.

We now define $\Lambda=\Phi_{L_{1}}$ on $(L \log L)_{+}$. It is trivial to show that for $1<$ $p<\infty$ we have $\mathscr{I}_{L_{p}}=(L \log L)_{+}$and $\Phi_{L_{p}}=\frac{1}{p} \Lambda$. For $p=\infty, \mathscr{I}_{L_{\infty}}=L_{1}^{+}$, and $\Phi_{L_{\infty}}=0$.

In order to describe the properties of indicators we introduce some further definitions. Let $\mathscr{I}$ be any semi-ideal in $L_{!}^{+}$and let $\Phi: \mathscr{I} \rightarrow \mathbf{C}$ be a map. We say that $\Phi$ is semilinear if

(4.4) $\Phi(\alpha f)=\alpha \Phi(f)$ for $f \in \mathscr{I}, \alpha \geq 0$.

(4.5) There exists a constant $\delta<\infty$ so that for $f, g \in \mathscr{I}$,

$$
\left|\Delta_{\Phi}(f, g)\right| \leq \delta(\|f\|+\|g\|) .
$$

The least such constant $\delta$ is denoted by $\delta(\Phi)$.

(4.6) If $f \in \mathscr{I}$ and $0 \leq f_{n} \leq f$ with $\lim _{n \rightarrow \infty}\left\|f_{n}\right\|=0$ then $\lim _{n \rightarrow \infty} \Phi\left(f_{n}\right)=$ 0 . if

We call $\Phi$ real if it takes only real values. We further say that $\Phi$ is continuous

$$
\lim _{\varepsilon \rightarrow 0} \sup _{\|f\| \leq 1} \sup _{\|g\| \leq \varepsilon}\left|\Delta_{\Phi}(f, g)\right|=0,
$$

and convex if

$$
\Delta_{\Phi}(f, g) \geq 0, \quad f, g \in \mathscr{I} .
$$


Proposition 4.2. Let $X$ be a g-convex function space. Then $\mathscr{I}_{X}$ is a strict semiideal and $\Phi_{X}$ is a continuous, convex real semilinear map. If $X$ is a Köthe function space then $\delta\left(\Phi_{X}\right) \leq \log 2$.

Proof. First of all, we observe that for some $\alpha>0, X^{\alpha}$ is isomorphic to a Köthe function space. Thus any $f \in\left(X^{\alpha}\right)^{*} \cap L_{1}$ satisfies

$$
\sup _{x \in B_{X}} \int f \log _{+}|x| d \mu<\infty \text {. }
$$

If we then pick some fixed strictly positive $x_{0} \in B_{X}$, then there is a strictly positive $f \in\left(X^{\alpha}\right)^{*} \cap L_{1}$ for which $f|\log | x_{0} \|$ is integrable. Thus $\mathscr{I}_{X}$ is a strict semi-ideal.

Now conditions (4.4) and (4.8) are obvious. Let us prove (4.5) and (4.7). Recall the definition of $\eta_{X}(\tau)$ from $\S 3$. Then if $f, g \in \mathscr{J}_{X}$ with $\|f\| \leq 1$, $\|g\| \leq \tau$, we have for any $\varepsilon>0, x, y \in B_{X}$ with

$$
\int f \log |x| d \mu \geq \Phi_{X}(f)-\frac{\varepsilon}{2}, \quad \int g \log |y| d \mu \geq \Phi_{X}(g)-\frac{\varepsilon}{2} .
$$

Let $v=\eta_{X}(\tau)^{-1}(\max (|x|, \tau|y|))$. Then $v \in B_{X}$ and

$$
\int(f+g) \log v d \mu \geq \Phi_{X}(f)+\Phi_{X}(g)-\varepsilon-\log \eta_{X}(\tau)\|f\|-\log \frac{\eta_{X}(\tau)}{\tau}\|g\|
$$

so that

$$
\Delta_{\Phi_{X}}(f, g) \leq(1+\tau) \log \eta_{X}(\tau)+\tau \log \frac{1}{\tau} .
$$

As $\lim _{\tau \rightarrow 1} \eta_{X}(\tau)=1$ this implies (4.5) and (4.7). If $X$ is a Köthe function space then $\eta_{X}(\tau) \leq 1+\tau$ so that $\Delta_{\Phi_{X}}(f, g) \leq(1+\tau) \log (1+\tau)-\tau \log \tau \leq$ $\log 2(1+\tau)$ provided $0 \leq \tau \leq 1$ which establishes that $\delta\left(\Phi_{X}\right) \leq \log 2$.

It remains to establish (4.6). Assume $f \in \mathscr{J}_{X}$ and $0 \leq f_{n} \leq f$ with $\left\|f_{n}\right\| \rightarrow$ 0 . If $x \in B_{X}$ is chosen so that $f \log |x|$ is integrable, then clearly by an application of the Dominated Convergence Theorem we have $\lim \int f_{n} \log |x| d \mu=0$ and hence $\liminf \Phi_{X}\left(f_{n}\right) \geq 0$.

To complete the proof we introduce a Köthe function space $F$ defined by

$$
\|g\|_{F}=\sup _{x \in B_{X}} \int|g| \log _{+}|x| d \mu+\|g\| .
$$

We claim that the norm on $F$ is order continuous. To prove this it suffices to take $g \in F_{+}$and a disjoint sequence $A_{n} \in \Sigma$ and show that $\left\|g 1_{A_{n}}\right\|_{F} \rightarrow 0$. In fact, if not, passing to a subsequence if necessary, we can find $x_{n} \in B_{X}$ with

$$
\int_{A_{n}} g \log _{+}\left|x_{n}\right| d \mu \geq \varepsilon>0
$$

for suitable $\varepsilon$. Let $y_{n}=\max \left(\left|x_{1}\right|, \ldots,\left|x_{n}\right|\right)$. For suitable $p>0$ and $\beta<\infty$ we have $\left\|y_{n}\right\|_{X} \leq \beta n^{1 / p}$ and hence $\beta^{-1} n^{-1 / p} y_{n} \in B_{X}$. However,

$$
\int g \log _{+} y_{n} d \mu \geq n \varepsilon-\left(\log \beta+\frac{1}{p} \log n\right)\|g\|,
$$

which leads to a contradiction. 0 .

Returning to the proof we obtain $\left\|f_{n}\right\|_{F} \rightarrow 0$ and so $\lim \sup _{n \rightarrow \infty} \Phi_{X}\left(f_{n}\right) \leq$ 
Lemma 4.3. Let $\mathscr{I}$ be a semi-ideal and let $\Phi$ be a semilinear functional on $\mathcal{I}$. Then

(i) For every $f \in \mathscr{I}, \Phi$ is bounded on $[0, f]$.

(ii) If $f_{n} \in[0, f]$ and $\lim \left\|f_{n}-g\right\|=0$ then $\lim \sup _{n \rightarrow \infty}\left|\Phi\left(f_{n}\right)-\Phi(g)\right| \leq$ $2 \delta(\Phi)\|f\|$.

(iii) If $\Phi$ is continuous then $\Phi$ is continuous on $[0, f]$ for the $L_{1}$-norm.

Proof. (i) For $f \in \mathscr{I}$, let $\Gamma(f)=\sup \{|\Phi(g)|: 0 \leq g \leq f\}$. Then $\Gamma(f+g) \leq$ $\Gamma(f)+\Gamma(g)+\delta\|f+g\|$ where $\delta=\delta(\Phi)$. Suppose $\Gamma(f)=\infty$. Then there exists $g \in[0, f]$ with $|\Phi(g)|>3|\Phi(f)|+\delta\|f\|+1$. Thus $|\Phi(f-g)|>2|\Phi(f)|+1$. Arguing by induction, there exists a descending sequence $f_{n}$ with $f_{0}=f$, $\Gamma\left(f_{n}\right)=\infty$, and $\left|\Phi\left(f_{n}\right)\right| \rightarrow \infty$. Let $g=\lim f_{n}$. Then

$$
\left|\Phi\left(f_{n}\right)\right| \leq|\Phi(g)|+\left|\Phi\left(f_{n}-g\right)\right|+\delta\|f\| .
$$

As $\lim \Phi\left(f_{n}-g\right)=0$ this yields a contradiction.

(ii) Let $h_{n}=\max \left(f_{n}, g\right)$. Then

$\left|\Phi(h, n)-\Phi\left(f_{n}\right)-\Phi\left(h_{n}-f_{n}\right)\right| \leq \delta\|f\|, \quad\left|\Phi\left(h_{n}\right)-\Phi(g)-\Phi\left(h_{n}-g\right)\right| \leq \delta\|f\|$.

Thus

$$
\left|\Phi\left(f_{n}\right)-\Phi(g)\right| \leq\left|\Phi\left(h_{n}-f_{n}\right)\right|+\left|\Phi\left(h_{n}-g\right)\right|+2 \delta\|f\|
$$

and since $\lim \Phi\left(h_{n}-f_{n}\right)=\lim \Phi\left(h_{n}-g\right)=0$ (ii) follows.

(iii) In this case, arguing as in case (ii)

$$
\lim _{n \rightarrow \infty}\left|\Delta_{\Phi}\left(f_{n}, h_{n}-f_{n}\right)\right|=\lim _{n \rightarrow \infty}\left|\Delta_{\Phi}\left(g, h_{n}-g\right)\right|=0
$$

and so $\lim \left|\Phi\left(f_{n}\right)-\Phi(g)\right|=0$.

Theorem 4.4. Let $X$ be a $g$-convex function space and suppose $\mathcal{I}$ be a strict semi-ideal contained in $\mathscr{I}_{X}$. Then $x \in B_{X}$ if and only if $f \log _{+}|x|$ is integrable for every $f \in \mathscr{I}$ and

$$
\int f \log |x| d \mu \leq \Phi_{X}(f), \quad f \in \mathscr{F} .
$$

Proof. One direction is trivial. For the other, we first prove that we may suppose $\mathscr{I}=\mathscr{I}_{X}$. Indeed suppose $f \in \mathscr{I}_{X}$ and that $f_{n} \in \mathscr{I}_{X}$ with $0 \leq f_{n} \uparrow f$ a.e. Let $A=\{s:|x(s)|>1\}$. Then $\int f_{n} 1_{A} \log |x| d \mu \leq \Phi_{X}\left(f_{n} 1_{A}\right)$. Now by the Monotone Convergence Theorem, Proposition 4.2, and Lemma 4.3 we have $\int f 1_{A} \log |x| d \mu \leq \Phi_{X}\left(f 1_{A}\right)$ so that $f \log _{+}|x| \in L_{1}$. Now if $f \log |x|$ fails to be integrable then $\int f \log |x| d \mu=-\infty<\Phi_{X}(f)$. Otherwise, we have

$$
\int f \log |x| d \mu=\lim _{n \rightarrow \infty} \int f_{n} \log |x| d \mu \leq \lim _{n \rightarrow \infty} \Phi_{X}\left(f_{n}\right)=\Phi_{X}(f)
$$

again by Lemma 4.3. Thus we will assume $\mathscr{I}=\mathscr{F}_{X}$.

To continue the proof assume $x \notin B_{X}$. Let $u$ be a strictly positive member of $B_{X}$ and let $w$ be a strictly positive function such that

$$
\int w(1+|\log | x||+|\log u|)^{2} d \mu<\infty .
$$

Let $L_{2}(w d \mu)$ be the corresponding weighted Hilbert space of all $\phi \in L_{0}$ such that $\|\phi\|_{w, 2}^{2}=\int|\phi|^{2} w d \mu<\infty$. Let $V$ be the subset of $L_{2}(w d \mu)$ of all functions of the form $\log |y|$ where $y \in B_{X}$. We observe that $V$ is convex 
(since $X$ is $g$-convex) and closed in the relative $L_{0}$-topology on $L_{2}(w d \mu)$ and hence closed in $L_{2}(w d \mu) . V$ is nonempty since $\log u \in V$. Further if $v \in V$ and $v_{1} \leq v$ a.e. with $v_{1} \in L_{2}(w d \mu)$ then $v_{1} \in V$.

By assumption, $\log |x| \notin V$. Thus by the Hahn-Banach theorem there exists $f \in L_{2}\left(w^{-1} d \mu\right)$ and $\alpha \in \mathbf{R}$ so that $\int f \log |x| d \mu>\alpha$ but $\int f \phi d \mu \leq \alpha$ for $\phi \epsilon$ $V$. From the properties of $V$, we must have $f \geq 0$ a.e. Since $\int|f|^{2} w^{-1} d \mu<$ $\infty$ and $w$ is integrable we have $f \in L_{1}$ by Hölder's inequality.

We show $f \in \mathscr{I}_{X}$. Assume $y \in B_{X}$, and let $y_{m, \tau}=\max (\min (|y|, m|x|), \tau u)$ for $m \in \mathbf{N}, \tau>0$. Then $\eta_{X}(\tau)^{-1} y_{m, \tau} \in B_{X}$ and $\log y_{m, \tau}-\log \eta_{X}(\tau) \in$ $L_{2}(w d \mu)$. Hence

$$
\int f \log y_{m, \tau} d \mu \leq \alpha+\|f\| \log \eta_{X}(\tau) .
$$

If we set $A=\{s:|y(s)|>1\}$ then

$$
\int_{A} f \log y_{m, 1} d \mu \leq \alpha+\|f\| \log \eta_{X}(1)+\int_{S \backslash A} f \log \frac{1}{|u|} d \mu,
$$

and letting $m \rightarrow \infty$

$$
\int f \log _{+}|y| d \mu=\int_{A} f \log |y| d \mu \leq \alpha+\|f\| \log \eta_{X}(1)+\int f|\log u| d \mu .
$$

Again

$$
\int f|\log u| d \mu \leq\|f\|_{w^{-1}, 2}\left(\int|\log u|^{2} w d \mu\right)^{1 / 2}
$$

so that we may conclude that $\sup _{y \in B_{X}} \int f \log _{+}|y| d \mu<\infty$. We also observe that (4.9) implies that for arbitrary $y \in B_{X}$

$$
\int f \log \left(\max (|y|, \tau u) d \mu \leq \alpha+\|f\| \log \eta_{X}(\tau)\right.
$$

for any $\tau>0$. Hence

$$
\int f \log |y| d \mu \leq \alpha+\|f\| \log \eta_{X}(\tau)
$$

Letting $\tau \rightarrow 0$ we have $\Phi_{X}(f) \leq \alpha$. Since $\int f \log |x| d \mu>\alpha$, this contradiction proves the theorem.

Our next proposition summarizes a few simple properties of the indicator.

Proposition 4.5. (i) Let $X, Y$ be g-convex function spaces and $\mathscr{I}$ be a strict semi-ideal contained in $\mathscr{I}_{X} \cap \mathscr{I}_{Y}$. Then $\|x\|_{X} \leq M\|x\|_{Y}$ for all $x \in L_{0}$ if and only if $\Phi_{X}(f) \geq \Phi_{Y}(f)-(\log M)\|f\|$ for all $f \in \mathcal{I}$. Further, $X$ and $Y$ have equivalent quasinorms if and only if

$$
d\left(\Phi_{X}, \Phi_{Y}\right)=\sup _{\|f\| \leq 1}\left|\Phi_{X}(f)-\Phi_{Y}(f)\right|<\infty .
$$

(ii) If $Z=X^{\alpha} Y^{\beta}$ (i.e., $\left.\|x\|_{Z}=\inf \left\{\|u\|_{X}^{\alpha}\|v\|_{Y}^{\beta}:|x| \leq|u|^{\alpha}|v|^{\beta}\right\}\right)$, then $\mathscr{I}_{Z} \supset$ $\mathscr{I}_{X} \cap \mathscr{I}_{Y}$ and

for $f \in \mathscr{I}_{X} \cap \mathscr{I}_{Y}$.

$$
\boldsymbol{\Phi}_{Z}(f)=\alpha \boldsymbol{\Phi}_{X}(f)+\beta \boldsymbol{\Phi}_{Y}(f)
$$

We omit the proofs. Part (i) is almost immediate from the definitions and Theorem 4.4, while (ii) is left as an easy exercise. We shall call two semilinear 
functionals $\Phi, \Psi$ defined on some strict semi-ideal $\mathscr{I}$ equivalent if $d(\Phi, \Psi)<$ $\infty$ where $d(\Phi, \Psi)$ is defined above.

Example. If we take $X=L(p, \infty)$ then it is relatively easy to compute

$$
\Phi_{X}(f)=\sup _{x \in B_{X}} \int f \log |x| d \mu=\frac{1}{p} \int_{0}^{\infty} f^{*}(t) \log t d t
$$

for $f \in L \log L$ where $f^{*}$ is the decreasing rearrangement of $f$. However $X$ is not $g$-convex, even though $\Phi_{X}$ is continuous and convex. In fact $\Phi_{X}=\Phi_{\widetilde{X}}$ where $\tilde{X}$ is the " $g$-convexification" of $X$ i.e., $x \in B_{X}$ if and only if

$$
\int_{0}^{\tau} \log x^{*}(t) d t \leq \frac{1}{p} \int_{0}^{\tau} \log \frac{1}{t} d t
$$

Clearly \|\|$_{\widetilde{X}}$ is an equivalent quasinorm on $L(p, \infty)$ since $L(p, \infty)$ is isomorphic to a $g$-convex space.

Proposition 4.6. Let $X$ be a Köthe function space. Then $\mathscr{I}_{X} \cap \mathscr{J}_{X^{*}} \subset(L \log L)_{+}$ and for $f \in \mathscr{I}_{X} \cap \mathscr{I}_{X^{*}}$, we have $\Phi_{X}(f)+\Phi_{X^{*}}(f)=\Lambda(f)$.

Furthermore if $f \in \mathscr{I}_{X} \cap \mathscr{I}_{X^{*}}$, and $\|f\|=1$, then $\Phi_{X}(f)=\int f \log x d \mu$ where $f=x y$ is the Lozanovskii factorization for $\left(X, X^{*}\right)$ i.e., $x, y \geq 0$, $\|x\|_{X}=\|y\|_{X^{*}}=1$, and $\operatorname{supp} x=\operatorname{supp} y=\operatorname{supp} f$.

Proof. The first part is essentially Lozanovskii's theorem that $B_{X} \cdot B_{X^{*}}=B_{L_{1}}$. For the second suppose $\xi \in B_{X}$. Then

$$
\begin{aligned}
\int f \log |\xi| d \mu & =\int f \log |\xi y| d \mu-\int f \log |y| d \mu \\
& \leq \int f \log f d \mu-\int f \log |y| d \mu \\
& =\int f \log x d \mu .
\end{aligned}
$$

We conclude this section by generalizing the fact which we have already noticed that $\boldsymbol{\Phi}_{X^{\theta} Y^{1-\theta}}(f)=\theta \Phi_{X}(f)+(1-\theta) \Phi_{Y}(f)$ for all $f \in \mathscr{I}_{X} \cap \mathscr{I}_{Y}$.

Theorem 4.7. Let $\mathscr{X}=\left(X_{w}: w \in \mathbf{T}\right)$ be a strongly admissible family of Köthe function spaces. Then there is a strict semi-ideal $\mathscr{I}$ so that if $f \in \mathscr{I}, \Phi_{X_{\text {eit }}}(f)$ is a bounded measurable function of $t$ and for $z=\dot{r} e^{i \theta} \in D$ we have $f \in \mathscr{I}_{X_{z}}$ with

$$
\Phi_{X_{z}}(f)=\int_{-\pi}^{\pi} P(r, \theta-t) \Phi_{X_{e i t}}(f) \frac{d t}{2 \pi} .
$$

Proof. There exist strictly positive $h, k$ so that for a.e. $w \in \mathbf{T},\|x h\|_{1} \leq$ $\|x\|_{X_{w}} \leq\|x k\|_{\infty}$. Let $\mathscr{I}=\mathscr{I}_{L_{1}(h d u)} \cap \mathscr{I}_{L_{1}\left(k^{-1} d\right)}$. Then $\mathscr{I} \subset \mathscr{I}_{X_{w}}$ for a.e. $w \in \mathbf{T}$. We also observe that it suffices to establish the formula for the case $z=0$ by a conformal mapping argument.

Consider, as in $\S 3$, the Köthe function space $\mathscr{E}$ on $(S \times \mathbf{T}, \mu \times \lambda)$ given by $\|\phi\|_{\mathscr{E}}=\operatorname{ess} \sup \left\|\phi\left(\cdot, e^{i \theta}\right)\right\|_{X_{e i \theta}}$. Now if $f \in \mathscr{I}$ with $\|f\|=1$, then by Lozanovskii's theorem there is a factorization $f(s)=\phi_{1}\left(s, e^{i t}\right) \phi_{2}\left(s, e^{i t}\right)$ with $\phi_{1}, \phi_{2} \geq 0, \operatorname{supp} \phi_{1}=\operatorname{supp} \phi_{2}=\operatorname{supp} f \times \mathbf{T}$, and $\left\|\phi_{1}\right\|_{\mathscr{E}}=\left\|\phi_{2}\right\|_{\mathscr{E}} * 1$.

Define $x \in X_{0}$ by

$$
x(s)=\exp \left(\int_{-\pi}^{\pi} \log \phi_{1}\left(s, e^{i t}\right) \frac{d t}{2 \pi}\right) .
$$


As discussed in $\S 3$, this is well defined since $\log _{+} \phi_{1}\left(s, e^{i t}\right)$ is integrable for a.e. $s \in S$. Similarly

$$
\int_{-\pi}^{\pi} \int_{S} \phi_{2}\left(s, e^{i t}\right) k(s)^{-1} d \mu(s) \frac{d t}{2 \pi} \leq 1
$$

so that $\log _{+} \phi_{2}\left(s, e^{i t}\right)$ is integrable for a.e. $s \in S$ and we may define

$$
y(s)=\exp \left(\int_{-\pi}^{\pi} \log \phi_{2}\left(s, e^{i t}\right) \frac{d t}{2 \pi}\right) .
$$

Now suppose $\xi \in B_{X_{0}}$ and $\xi \geq 0$. Then there exists $\psi \in B_{\mathscr{C}}$ with

$$
\xi(s)=\exp \left(\int_{-\pi}^{\pi} \log \psi\left(s, e^{i t}\right) \frac{d t}{2 \pi}\right) .
$$

Thus

$$
\begin{aligned}
\int_{S} y \xi d \mu & =\int_{S} \exp \left(\int_{-\pi}^{\pi} \log \left(\phi_{2} \psi\right) \frac{d t}{2 \pi}\right) d \mu \\
& \leq \int_{S} \int_{-\pi}^{\pi} \phi_{2} \psi \frac{d t}{2 \pi} d \mu \\
& \leq\left\|\phi_{2}\right\|_{\mathscr{E}}\|\psi\|_{\mathscr{E}} \leq 1 .
\end{aligned}
$$

Thus $\|y\|_{X_{0}^{*}} \leq 1$. However $x y=f$, so this is the Lozanovskii factorization of $f$ and

$$
\Phi_{X_{0}}(f)=\int f \log x d \mu=\int_{S} \int_{-\pi}^{\pi} f \log \phi_{1}\left(s, e^{i t}\right) \frac{d t}{2 \pi} d \mu(s) .
$$

As in $\S 3$, we have

$$
\left\|\phi_{2}\right\|_{\mathscr{E} *}=\int_{-\pi}^{\pi}\left\|\phi_{2}\left(\cdot, e^{i t}\right)\right\|_{X_{e^{*}}^{* i}} \frac{d t}{2 \pi}
$$

and as $\phi_{1} \phi_{2}=f \otimes 1_{\mathbf{T}}$ we must have $\left\|\phi_{2}\left(\cdot, e^{i t}\right)\right\|_{X_{e^{i t}}^{*}} \geq 1$ a.e. So we have $\left\|\phi_{2}\left(\cdot, e^{i t}\right)\right\|_{X_{e^{*}}}=1$ a.e. Thus $\lambda$-a.e. $f(s)=\phi_{1}\left(s, e^{i t}\right) \phi_{2}\left(s, e^{i t}\right)$ is the Lozanovskii factorization of $f$ for the pair $X_{e^{i t}}, X_{e^{i t}}^{*}$. Hence

$$
\Phi_{X_{e^{i t}}}(f)=\int f \log \phi_{1}\left(s, e^{i t}\right) d \mu(s)
$$

and the theorem follows.

Corollary 4.8. We have the duality theorem that the space $X_{z}^{*}$ obtained by interpolating $\mathscr{X}^{*}=\left\{X_{w}^{*}: W \in \mathbf{T}\right\}$ is isometric to $\left(X_{z}\right)^{*}$.

This is immediate from 4.6 and 4.7.

Corollary 4.9. If $f \in\left(L_{1}\right)_{+}$with $\|f\|=1$ has a Lozanovskii factorization $f=x x^{*}$ for $X_{z}^{*}$ where $|z|<1$, and if $|u|=1$ a.e., then there is a unique $F \in \mathscr{N}^{+}$, with $F(z)=u x, F$ vanishes identically off the support of $x$ and $\|F\|_{\mathscr{X}}=1$. Hence the induced centralizer $\Omega$ is uniquely defined at ux.

Proof. Let $G$ be an extremal for $u^{-1} x^{*}$ in $\mathscr{X}^{*}$, i.e., suppose $G \in \mathcal{N}^{+}$, $G(z)=u^{-1} x^{*}$, and $\|G\|_{\mathscr{X}}=1$. If $F$ is an extremal for $u x$ in $\mathscr{X}$, then it follows from the maximum modulus principle that since $\int F(z) G(z) d \mu=1$ then $\int F(\zeta) G(\zeta) d \mu=1$ for all $|\zeta|<1$. Hence $F(\zeta) G(\zeta)$ is real and nonnegative a.e. Thus for a.e. $s F(\zeta, s) G(\zeta, s)$ is real on a dense subset of the disk 
and hence everywhere. Thus $F(\zeta) G(\zeta)=f$ for all $\zeta$ and it follows easily that $F$ is uniquely determined on the support of $x$. This implies that $\Omega(u x)$ is also uniquely determined.

\section{Characterizing INDicators}

Theorem 5.1. Let $\mathcal{I}$ be a strict semi-ideal, and let $\Phi$ be a continuous convex real semilinear map defined on $\mathscr{I}$. Then there is a (unique) $g$-convex function space $X$ for which $\mathscr{I} \subset \mathscr{I}_{X}$ and $\Phi(f)=\Phi_{X}(f)$ for $f \in \mathscr{I}$.

Proof. Let $B_{X}$ be the subset of $L_{0}$ of all $x$ such that $f \log _{+}|x| \in L_{1}$ for any $f \in \mathscr{J}$ and $\int f \log |x| d \mu \leq \Phi(f)$ for $f \in \mathscr{J}$. We define $\|x\|_{X}=\inf \{\alpha>0$ : $\left.\alpha^{-1}|x| \in B_{X}\right\}$ and $\|x\|_{X}=\infty$ if $x \notin \alpha B_{X}$ for every $\alpha>0$. Clearly $B_{X}=$ $\left\{x:\|x\|_{X} \leq 1\right\}$.

We now show that \|\|$_{X}$ defines a $g$-convex quasinorm. Suppose $x, y \in B_{X}$ with disjoint supports contained in $A_{x}, A_{y}$ where $A_{x} \cup A_{y}=S$ and $A_{x} \cap A_{y}=$ $\varnothing$. For $f \in \mathscr{I}$,

$$
\begin{aligned}
\int f \log (\max (|x|, \tau|y|)) d \mu & \leq \Phi\left(f 1_{A_{x}}\right)+\Phi\left(f 1_{A_{y}}\right)+\log \tau\left\|f 1_{A_{y}}\right\| \\
& \leq \Phi(f)+\Delta_{\Phi}\left(f 1_{A_{x}}, f 1_{A_{y}}\right)+\log \tau\left\|f 1_{A_{y}}\right\|
\end{aligned}
$$

Let

$$
\sigma(\varepsilon)=\sup _{\|f\| \leq 1} \sup _{\|g\| \leq \varepsilon}\left|\Delta_{\Phi}(f, g)\right|
$$

Then

$$
\int f \log (\max (|x|, \tau|y|)) d \mu \leq \Phi(f)+\|f\|(\sigma(\alpha)+\alpha \log \tau),
$$

where $\alpha=\left\|f 1_{A_{y}}\right\| /\|f\|$. If we let

$$
\sigma^{*}(\tau)=\sup _{0 \leq \alpha \leq 1}(\sigma(\alpha)+\alpha \log \tau)
$$

then $\lim _{\tau \rightarrow 0} \sigma^{*}(\tau)=0$ and

$$
\int f \log (\max (|x|, \tau|y|)) d \mu \leq \Phi(f)+\|f\| \sigma^{*}(\tau) .
$$

Thus $\|\max (|x|, \tau|y|)\|_{X} \leq e^{\sigma^{*}(\tau)}$ or $\eta_{X}(\tau) \leq e^{\sigma^{*}(\tau)}$.

To complete the proof that $X$ is a $g$-convex function space we need only show that $B_{X}$ contains a strictly positive function and that $B_{X}$ is closed in $L_{0}$, since condition (3.5) is trivial. Let us postpone the former statement and simply show that $B_{X}$ is $L_{0}$-closed. Suppose $x_{n} \in B_{X}^{+}$and $x_{n} \rightarrow x$ a.e. We may suppose, without loss of generality that $\operatorname{supp} x_{n} \subset \operatorname{supp} x=A$ for every $n$. Let $A_{k}$ be an increasing sequence of measurable subsets of $A$ so that $\mu\left(A \backslash \bigcup A_{k}\right)=0$ and $x_{n} x^{-1} \rightarrow 1$ uniformly on each $A_{k}$. Then, given $\varepsilon>0$, $k \in \mathbf{N}$, there exists $N \in \mathbf{N}$ so that if $n \geq N, x_{n} 1_{A_{k}} \geq(1-\varepsilon) x 1_{A_{k}}$. Hence if $f \in \mathscr{I}, f \log _{+} x 1_{A_{k}} \in L_{1}$ and

$$
\begin{aligned}
\int f \log x 1_{A_{k}} d \mu & \leq-\log (1-\varepsilon)\|f\|+\int f \log x_{n} 1_{A_{k}} d \mu \\
& \leq-\log (1-\varepsilon)\|f\|+\Phi(f) .
\end{aligned}
$$


If we let $\varepsilon \rightarrow 0$ we obtain that $x 1_{A_{k}} \in B_{X}$ for every $k \in \mathbf{N}$. Now let $G=$ $\{s: x(s)>1\}$. If $f \in \mathscr{I}$,

$$
\begin{aligned}
\int f \log _{+} x d \mu & =\int_{G} f \log x d \mu=\lim _{k \rightarrow \infty} \int_{G \cap A_{k}} f \log x d \mu \\
& \leq \liminf _{k \rightarrow \infty} \Phi\left(f 1_{G \cap A_{k}}\right)=\Phi\left(f 1_{G}\right),
\end{aligned}
$$

using Lemma 4.3 and the fact that $\Phi$ is continuous. Hence $f \log _{+} x \in L_{1}$. If $f \log x \in L_{1}$ then the same argument yields that $\int f \log x d \mu \leq \Phi(f)$ and this also holds in the case when $f \log x$ fails to be integrable. Thus $x \in B_{X}$ and $B_{X}$ is closed in $L_{0}$.

To conclude we will check that $B_{X}$ contains a strictly positive element and that $\Phi=\Phi_{X}$ on $\mathscr{I}$. Notice first that if $f \in \mathscr{J}, x \in B_{X}$, then

$$
\int f \log _{+}|x| d \mu \leq \Phi\left(f 1_{|x|>1}\right) \leq \sup _{g \in[0, f]} \Phi(g)<\infty
$$

by Lemma 4.3 . Hence

$$
\sup _{x \in B_{X}} \int f \log _{+}|x| d \mu<\infty \text {. }
$$

Now suppose $f \in \mathscr{I}$ and $\Phi(f)>\alpha$. Then we show the existence of $x \in B_{X}$ so that $\int f \log |x| d \mu<\alpha$. This will imply, using some strictly positive $f$ that $B_{X}$ contains a strictly positive function and hence $X$ is a $g$-convex function space. Further, from the above remarks we will have that $\mathscr{I} \subset \mathscr{I}_{X}$ and we will be able to conclude that $\Phi_{X}=\Phi$ on $\mathscr{J}$.

Let $Y$ be the AM-space $\bigcup n[-f, f]$ normed by $\|g\|_{Y}=$ ess sup $|g(s)| f(s)^{-1}$. Let $K \subset Y^{+}$be the convex set of all $g \geq 0$ such that $\Phi(g) \leq \alpha$. For $m \in \mathbf{N}$, $K \cap m[0, f]$ is closed for convergence in $L_{1}$-norm by Lemma 4.3 and the continuity of $\Phi$. Let $Y_{*}$ be the predual of $Y$, i.e. the space of all measurable functions $h$ with $\operatorname{supp} h \subset \operatorname{supp} f$ such that $\|h\|_{Y_{*}}=\int|h| f d \mu<\infty$. Then $K \cap m[-f, f]$ is closed for the weak * topology $\sigma\left(Y, Y_{*}\right)$ which is weaker on $m[-f, f]$ than the $L_{1}$-norm. Now by the Banach-Dieudonne theorem, $K$ is $\sigma\left(Y, Y_{*}\right)$-closed in $Y$. Thus there exists $h \in Y_{*}$, so that $\int h \phi \leq \alpha$ for $\phi \in K$ but $\int h f d \mu>\alpha$. Clearly if $g \in Y, g \geq 0, \int h g d \mu \leq \Phi(g)$.

Let $x(s)=e^{h(s)}$ for $s \in \operatorname{supp} f$ and $x(s)=0$ otherwise. Then if $g \in \mathscr{I}$

$$
\begin{aligned}
\int g \log _{+} x d \mu & =\int g h d \mu=\lim _{n \rightarrow \infty} \int_{h>0} \min (g, n f) h d \mu \\
& \leq \liminf _{n \rightarrow \infty} \Phi\left(\min \left(g 1_{(h>0)}, n f\right)\right) \\
& =\Phi\left(g 1_{(h>0)}\right)
\end{aligned}
$$

by Lemma 4.3. If $\operatorname{supp} g$ is not a subset of $\operatorname{supp} f$ then $\int g \log x d \mu=-\infty$. Otherwise, if $g \log x$ is integrable

$$
\int g \log x d \mu=\int g h d \mu=\lim _{n \rightarrow \inf } \int \max (g, n f) h d \mu \leq \Phi(g)
$$

by the same reasoning. Hence we can conclude that $x \in B_{X}$ and the proof is complete.

Theorem 5.2. Let $\mathscr{J}$ be a strict semi-ideal and let $\Phi$ be a real semilinear functional on $\mathcal{I}$. Then, in order that there exist a Köthe function space $X$ with 
$\mathscr{I} \subset \mathscr{I}_{X}$, and $\Phi(f)=\Phi_{X}(f)$ for $f \in \mathscr{I}$, it is necessary and sufficient that for any $f, g \in \mathscr{I}$,

$$
0 \leq \Delta_{\Phi}(f, g) \leq \Delta_{\Lambda}(f, g) .
$$

Proof. Necessity is clear since $\Phi_{X^{*}}=\Lambda-\Phi_{X}$. Conversely, assume (5.1). Since $\Lambda$ is continuous, both $\Phi$ and $\Lambda-\Phi$ are convex and continuous. Thus there exist $g$-convex function spaces $X, Y$ with $\mathscr{I} \subset \mathscr{I}_{X} \cap \mathscr{I}_{Y}$ and so that $\Phi=\Phi_{X}$ and $\Lambda-\Phi_{X}=\Phi_{Y}$. It suffices to show that $X$ is a Köthe function space. Clearly $B_{X} \cdot B_{Y} \subset B_{L_{1}}$. Let $B_{\widetilde{X}}$ be the $L_{0}$-closed convex hull of $B_{X}$. Then $B_{\widetilde{X}} \cdot B_{Y} \subset B_{L_{1}}$, and so on $\mathscr{F}_{\widetilde{X}} \cap \mathscr{I}$ we have $\Phi_{X} \leq \Phi_{\widetilde{X}} \leq \Lambda-\Phi_{Y}=\Phi_{X}$. Hence by Theorem $4.4 \tilde{X}=X$ and the proof is completed.

Theorem 5.3. Let $X$ be a g-convex function space and let $\mathscr{I} \subset \mathscr{I}_{X} \cap(L \log L)_{+}$ be a strict semi-ideal. Then

(i) $X$ is p-convex $(0<p<\infty)$ if and only if for every $f, g \in \mathscr{I}$,

$$
\Delta_{\Phi_{X}}(f, g) \leq \frac{1}{p} \Delta_{\Lambda}(f, g) .
$$

(ii) If $X$ is p-convex for some $p>0$ then $X$ is also q-concave where $0<$ $q<\infty$ if and only if for every $f, g \in \mathscr{I}$,

$$
\Delta_{\Phi_{X}}(f, g) \leq \frac{1}{q} \Delta_{\Lambda}(f, g) .
$$

Proof. Part (i) is obvious. For (ii), suppose $Y=X^{p}$. Then $Y$ is a Köthe function space and $\Phi_{Y^{*}}=\Lambda-p \Phi_{X}$. Thus $\Delta_{\Phi_{Y^{*}}}(f, g)=\Delta_{\Lambda}(f, g)-p \Delta_{\Phi_{X}}(f, g)$.

Hence $Y^{*}$ is $r$-convex if and only if

$$
\Delta_{\Phi_{X}}(f, g) \geq\left(1-\frac{1}{r}\right) \frac{1}{p} \Delta_{\Lambda}(f, g)
$$

for $f, g \in \mathscr{I}$. Now $X$ is $q$-concave if and only if $Y$ is $q / p$-concave if and only if $Y^{*}$ is $(1-p / q)^{-1}$-concave. This yields the theorem.

Theorem 5.3 has a simple illustration in a theorem of Pisier [43].

Corollary 5.4 (Pisier). Suppose $X$ is a Köthe function space and $0<\theta<1$. In order that there is a Köthe function space $Y$ with $X=L_{2}^{\theta} Y^{1-\theta}$ it is necessary and sufficient that $X$ is p-convex and $q$-concave where $\frac{1}{q}=1-\frac{1}{p}=\frac{\theta}{2}$.

Proof. This is a simple calculation based on the fact that $Y$ can be determined from the equation $\Phi_{X}=\frac{\theta}{2} \Lambda+(1-\theta) \Phi_{Y}$ on a suitable semi-ideal together with the preceding two theorems.

We remark that some recent results of Cwikel and Nilsson [16] on complex interpolation between Banach lattices can also be proved by this method.

Lemma 5.5. For any Köthe function space $X$, if $f_{1}, \ldots, f_{n} \in \mathscr{I}_{X} \cap(L \log L)_{+}$ and $\sum_{i=1}^{n}\left\|f_{i}\right\|=1$ then

$$
\Delta_{\Phi_{X}}\left(f_{1}, \ldots, f_{n}\right) \leq \sum_{i=1}^{n}\left\|f_{i}\right\| \log \frac{1}{\left\|f_{i}\right\|} .
$$

In particular $\delta_{\Phi_{X}} \leq \delta_{\Lambda}=\log 2$. 
Proof. This follows immediately from the observation:

$$
\begin{aligned}
\Delta_{\boldsymbol{\Phi}_{X}}\left(f_{1}, \ldots, f_{n}\right) & \leq \Delta_{\Lambda}\left(f_{1}, \ldots, f_{n}\right) \\
& =\sum_{i=1}^{n} \int f_{i} \log \frac{f_{i}}{F} d \mu+\sum_{i=1}^{n}\left\|f_{i}\right\| \log \frac{1}{\left\|f_{i}\right\|},
\end{aligned}
$$

where $F=f_{1}+\cdots+f_{n}$.

In the above proof that if $f_{1}, \ldots, f_{n}$ have disjoint supports then

$$
\Delta_{\Lambda}\left(f_{1}, \ldots, f_{n}\right)=-\sum\left\|f_{i}\right\| \log \left\|f_{i}\right\| .
$$

Lemma 5.6. Suppose $X$ is a Köthe function space and that $\mathcal{J}$ is the ideal generated by $\mathscr{I}_{X} \cap(L \log L)_{+}$(thus $\mathscr{J}$ consists of all complex $f \in L_{0}$ so that $\left.|f| \in \mathscr{I} \cap(L \log L)_{+}\right)$. Then extend $\Phi_{X}$ to $\mathscr{J}$ by $\Phi_{X}(f)=f \log x$ where $f=x y$ is the Lozanovskii factorization of $|f|$. Then for $f, g \in \mathcal{J}$,

$$
\Delta_{\mathbf{\Phi}_{X}}(f, g) \leq \frac{4}{e}(\|f\|+\|g\|) .
$$

Proof. First suppose $0 \leq g \leq f \in \mathscr{T}_{X} \cap(L \log L)_{+}$. Then if we let $f=x_{1} y_{1}$ and $g=x_{2} y_{2}$ be the corresponding Lozanovskii factorizations, we have

$$
\begin{aligned}
\Phi_{X}(g) & =\Lambda(g)-\int g \log \frac{\left|y_{2}\right|}{\|g\|} d \mu \\
& \leq \Lambda(g)-\int g \log \frac{\left|y_{1}\right|}{\|f\|} d \mu \\
& =\int g \log x_{1} d \mu+\|g\| \log \frac{\|f\|}{\|g\|} \\
& \leq \int g \log x_{1} d \mu+\frac{1}{e}\|f\| .
\end{aligned}
$$

If we then set $A=\left\{s: x_{1}(s)>x_{2}(s)\right\}$ we have

and

$$
\int_{A} g \log x_{1} d \mu \leq \Phi_{X}\left(g 1_{A}\right) \leq \int_{A} g \log x_{2} d \mu+\frac{1}{e}\|g\|,
$$

We conclude that

$$
\int_{S \backslash A} g \log x_{2} d \mu \leq \Phi_{X}\left(g 1_{s \backslash A}\right) \leq \int_{S \backslash A} g \log x_{1} d \mu+\frac{1}{e}\|f\| .
$$

whenever $0 \leq g \leq f$.

$$
\int g\left|\log x_{1}-\log x_{2}\right| d \mu \leq \frac{2}{e}\|f\|
$$

Now suppose $f, g$ are in $\mathscr{J}$ and let $h=|f|+|g|$. Let $h$ have the Lozanovskii factorization $h=\xi \eta$. Then by the above argument

$$
\left|\Phi_{X}(f)-\int f \log \xi d \mu\right| \leq \frac{2}{e}\|h\|
$$

with a similar inequality for $g$. Thus $\Delta_{\Phi_{X}}(f, g) \leq \frac{4}{e}\|h\|$ and the lemma follows.

We will refer to $\Phi_{X}$ extended in this way as the (extended) indicator of $X$.

We conclude this section with a result on the construction of Köthe function spaces which we will need later in $\S 9$. 
Theorem 5.7. Suppose $\mu$ is a nonatomic probability measure on $S$ and that $X$ is a Köthe function space such that $L_{\infty} \subset X \subset L_{p}$ for some $p>0$. Let $T: L_{1} \rightarrow L_{1}$ be a doubly stochastic operator, i.e., $T \geq 0, T 1_{S}=T^{*} 1_{S}=1_{S}$. Then there is a unique Köthe function space $Y$ such that $\Phi_{Y}(f)=\Phi_{X}(T f)$ whenever $f \in(L \log L)_{+}$.

Proof. We remark that $\mathscr{I}_{X} \supset L \log L$ and that if $f \in L \log L$ then $T f \in$ $L \log L$. We need the following lemma.

Lemma 5.8. Let $\left(a_{i j}\right)_{1 \leq i, j \leq n}$ be a doubly stochastic matrix. For any nonnegative $\left(x_{i}\right)_{i=1}^{n},\left(y_{i}\right)_{i=1}^{n}$, let $\xi_{i}=\sum_{j=1}^{n} a_{i j} x_{j}, \eta_{i}=\sum_{j=1}^{n} a_{i j} y_{j}$. We then have

$$
\sum_{i=1}^{n}\left(\xi_{i} \log \frac{\xi_{i}+\eta_{i}}{\xi_{i}}+\eta_{i} \log \frac{\xi_{i}+\eta_{i}}{\eta_{i}}\right) \leq \sum_{i=1}^{n}\left(x_{i} \log \frac{x_{i}+y_{i}}{x_{i}}+y_{i} \log \frac{x_{i}+y_{i}}{y_{i}}\right) .
$$

Proof of Lemma 5.8. We define for $(s, t) \in \mathbf{R}^{2}$ with $s, t \geq 0$,

$$
u(s, t)=s \log \frac{s+t}{s}+t \log \frac{s+t}{t} \text {. }
$$

Then by differentiation it can be seen that $u$ is a concave function on its cone of definition, since the second derivative is negative semidefinite in the interior. Now consider the function $v$ defined on the convex set of doubly stochastic matrices, given by

$$
v(A)=\sum_{i=1}^{n} u\left(\sum_{j=1}^{n} a_{i j} x_{j}, \sum_{j=1}^{n} a_{i j} y_{j}\right) .
$$

Then $v$ is also concave and assumes its maximum at an extreme point, i.e., a permutation matrix $S_{\sigma}=\left(\delta_{i, \sigma(j)}\right)$ where $\sigma$ is a permutation of $[1,2, \ldots, n]$. Then the lemma follows immediately.

Proof of Theorem 5.7. Let $\Phi(f)=\Phi_{X}(T f)$ for $f \in L \log L$. Let $D(n, k)$; $1 \leq k \leq 2^{n}, 1 \leq n$, be a family of measurable sets so that $D(0,1)=$ $S, D(n, k) \cap D\left(n, k^{\prime}\right)=\varnothing$ for $k \neq k^{\prime}, D(n, k)=D(n+1,2 k-1) \cup$ $D(n+1,2 k), \mu(D(n, k))=2^{-n}$ and the sets $(D(n, k))$ generate $\Sigma$. Let $E_{n}$ be the conditional expectation operator of $L_{1}$ onto $L_{1}\left(\Sigma_{n}\right)$ where $\Sigma_{n}$ is generated by $D(n, k)$ for $1 \leq k \leq 2^{n}$. Clearly the lemma implies that for any nonnegative $f, g \in L_{1}$, if $f_{n}=E_{n} f, g_{n}=E_{n} g$, and $\phi_{n}=E_{n} T E_{n} f$, $\psi_{n}=E_{n} T E_{n} g$,

$$
\begin{gathered}
\int \phi_{n} \log \frac{\phi_{n}+\psi_{n}}{\phi_{n}} d \mu+\int \psi_{n} \log \frac{\phi_{n}+\psi_{n}}{\psi_{n}} d \mu \\
\leq \int f_{n} \log \frac{f_{n}+g_{n}}{f_{n}} d \mu+\int g_{n} \log \frac{f_{n}+g_{n}}{g_{n}} d \mu .
\end{gathered}
$$

Since $\left\|f_{n}\right\|=\left\|\phi_{n}\right\|=\|f\|$ and $\left\|g_{n}\right\|=\left\|\psi_{n}\right\|=\|g\|$ this translates to $\Delta_{\Lambda}\left(\phi_{n}, \psi_{n}\right)$ $\leq \Delta_{\Lambda}\left(f_{n}, g_{n}\right)$.

If we now assume $f, g \in L \log L$ the continuity properties of $\Lambda$ on the Köthe function space $L \log L$ imply that $\Delta_{\Lambda}(T f, T g) \leq \Delta_{\Lambda}(f, g)$.

Now it follows that

$$
0 \leq \Delta_{\Phi}(f, g)=\Delta_{\Phi_{X}}(T f, T g) \leq \Delta_{\Lambda}(T f, T g) \leq \Delta_{\Lambda}(f, g) .
$$


Hence $\Phi$ satisfies the conditions of (4.4)-(4.6) and the theorem follows by Theorem 5.2.

It now follows easily from Theorem 5.7 that

Corollary 5.9. Under the hypotheses of Theorem 5.7 we have for any $f \in L \log L$,

$$
\left|\Phi_{Y}(f)-\Phi_{X}(T f)\right| \leq \frac{24}{e}\|f\| .
$$

Proof. Simply split each $f$ into real and imaginary and then positive and negative parts and use Theorem 5.7.

\section{APPROXIMATION THEOREMS FOR SEMILINEAR FUNCTIONALS}

Proposition 6.1. Let $\mathscr{I}$ be a strict semi-ideal contained in $(L \log L)_{+}$and let $\Phi$ be a semilinear functional on $\mathscr{I}$. Suppose that for some $M$ and every $f_{1}, \ldots, f_{n} \in \mathscr{I}$,

$$
-M \sum_{j=1}^{n}\left\|f_{j}\right\| \leq \Delta_{\Phi}\left(f_{1}, \ldots, f_{n}\right) \leq \Delta_{\Lambda}\left(f_{1}, \ldots, f_{n}\right)+M \sum_{j=1}^{n}\left\|f_{j}\right\| .
$$

Then, there exists a Köthe function space $X$ so that $\mathscr{I} \subset \mathscr{I}_{X}$ and

$$
d\left(\Phi, \Phi_{X}\right)=\sup _{\|f\| \leq 1}\left|\Phi(f)-\Phi_{X}(f)\right| \leq M+4 \delta,
$$

where $\delta=\delta(\Phi)$.

Proof. We first prove this in the special case when $S$ is a finite set and $\mathscr{J}=$ $L_{1}^{+}(S)$ is thus a closed cone in a finite dimensional space. Define $\Phi_{0}(f)=$ $\inf \sum_{j=1}^{n} \Phi\left(f_{j}\right)$ where the infimum is taken over all $f_{1}, \ldots, f_{n} \geq 0$ with $\sum_{j=1}^{n} f_{j}$ $=f$. Then $\Phi(f)-M\|f\| \leq \Phi_{0}(f) \leq \Phi(f)$ and $\Phi_{0}$ is a convex semilinear functional.

Let $P$ be the positive cone $\{x: x(s)>0 \forall s \in S\}$. We argue first that $\Phi_{0}$ is necessarily continuous on $P$. First notice that for some $K$ we have $\left|\Phi_{0}(f)\right| \leq K$ whenever $\|f\|_{\infty} \leq 1$. Now suppose $f \in P$ and $f_{n} \rightarrow f$. Then, given $\varepsilon>0$ there exists $N$ so that if $n \geq N,(1-\varepsilon) f \leq f_{n} \leq(1+\varepsilon) f$. Then $\left|\Phi_{0}\left((1+\varepsilon) f-f_{n}\right)\right|,\left|\Phi_{0}\left(f_{n}-(1-\varepsilon) f\right)\right| \leq 2 \varepsilon\|f\|_{\infty} K$ provided $n \geq N$. Thus

$$
\begin{aligned}
& (1+\varepsilon) \Phi_{0}(x) \leq \Phi_{0}\left(f_{n}\right)+2 \varepsilon\|f\|_{\infty} K, \\
& \Phi_{0}\left(f_{n}\right) \leq(1-\varepsilon) \Phi_{0}(f)+2 \varepsilon\|f\|_{\infty} K .
\end{aligned}
$$

We conclude that for $n \geq N,\left|\Phi_{0}(f)-\Phi_{0}\left(f_{n}\right)\right| \leq \varepsilon\left|\Phi_{0}(f)\right|+2 \varepsilon\|f\|_{\infty} K$, so that $\Phi_{0}$ is continuous on $P$.

Now let $B_{0}$ be the set of $x$ so that $\int f \log |x| d \mu \leq \Phi_{0}(f)$ whenever $f \in P$. Now a standard Hahn-Banach separation argument shows that for $f \in P$,

$$
\Phi_{0}(f)=\sup _{x \in B_{0}} \int f \log |x| d \mu .
$$

Arguing similarly, let $\Phi_{1}(f)=\inf \sum_{j=1}^{n}\left(\Lambda\left(f_{j}\right)-\Phi\left(f_{j}\right)\right)$ where the infimum is taken over all $f_{1}, \ldots, f_{n} \geq 0$ so that $\sum f_{i}=f$. Then by the same reasoning

$$
\Lambda(f)-\Phi(f)-M\|f\| \leq \Phi_{1}(f) \leq \Lambda(f)-\Phi(f),
$$


and if $B_{1}=\left\{x: \int f \log |x| d \mu \leq \Phi_{1}(f) \forall f \in P\right\}$ then for $f \in P$,

$$
\Phi_{1}(f)=\sup _{x \in B_{1}} \int f \log |x| d \mu .
$$

Since $\Phi_{0}+\Phi_{1} \leq \Lambda$ we conclude that $B_{0} \cdot B_{1} \subset B_{L_{1}}$. Let $B_{X}$ be the closed convex hull of $B_{0}$. Then $B_{X} \cdot B_{1} \subset B_{L_{1}}$ so that $\Phi_{X}(f)+\Phi_{1}(f) \leq \Lambda(f)$ for $f \in P$. Thus $\Phi(f)-M\|f\| \leq \Phi_{X}(f) \leq \Phi(f)+M\|f\|$ for every $f \in P$. In general if $f \geq 0$ then there is a sequence $f_{n} \downarrow f$ with $f_{n} \in P$. By Lemma 4.3 limsup $\left|\Phi\left(f_{n}\right)-\Phi(f)\right| \leq 2 \delta\|f\|$ and it follows that

$$
\left|\Phi(f)-\Phi_{X}\left(f_{n}\right)\right| \leq(M+2 \delta)\|f\|
$$

whenever $f \geq 0$.

We now turn to the general case. Let $\mathscr{A}=\left(A_{1}, \ldots, A_{n}\right)$ be any finite collection of disjoint measurable sets so that each $1_{A_{k}} \in \mathscr{I}$. Let $E_{\mathscr{A}}$ be the linear span of $1_{A_{k}}$ for $1 \leq k \leq n$. By the above there exists a semilinear map $\Psi_{\mathscr{A}}$ on $E_{\mathscr{A}}$ so that

$$
0 \leq \Delta_{\Psi_{\mathscr{A}}}(f, g) \leq \Delta_{\Lambda}(f, g), \quad f, g \in \mathscr{J}
$$

and

$$
\left|\Phi(f)-\Psi_{\mathscr{A}}(f)\right| \leq(M+2 \delta)\|f\|
$$

for $f \in \mathscr{I}$. By a simple compactness argument we can then determine a functional $\Psi$ on the cone $\mathscr{V}$ of simple functions in $\mathscr{I}$ so that

$$
0 \leq \Delta_{\Psi}(f, g) \leq \Delta_{\Lambda}(f, g), \quad|\Phi(f)-\Psi(f)| \leq(M+2 \delta)\|f\|
$$

for $f \in \mathscr{V}$.

Suppose $f \in \mathscr{I}$. Then $\mathscr{V} \cap[0, f]$ is dense in $[0, f]$ for the $L_{1}$-norm. We now use the reasoning of Lemma 4.3 to argue that $\Psi$ is uniformly continuous on $\mathscr{V} \cap[0, f]$. First notice that since $\Phi$ is semilinear, it follows that $|\Psi(g)| \leq$ $\eta_{0}(\|g\|)$ for $g \in \mathscr{V} \cap[0, f]$ where $\lim _{\varepsilon \rightarrow 0} \eta_{0}(\varepsilon)=0$. Suppose $g, h \in[0, f]$ and let $k=\max (g, h)$. Then if $\|g-h\| \leq \varepsilon$,

$$
|\Psi(g)-\Psi(h)| \leq \Delta_{\Psi}(g, k-g)\left|+\Delta_{\Psi}(h, k-h)\right|+|\Psi(k-g)|+|\Psi(k-h)| .
$$

Now

$$
\left|\Delta_{\Psi}(g, k-g)\right| \leq\left|\Delta_{\Lambda}(g, k-g)\right| \leq\|f\| \phi(\varepsilon),
$$

where $\lim _{\varepsilon \rightarrow 0} \phi(\varepsilon)=0$ since $\Lambda$ is a continuous semilinear functional. Similarly estimating the other terms we obtain that $|\Psi(g)-\Psi(h)| \leq \eta_{1}(\varepsilon)$, where $\lim _{\varepsilon \rightarrow 0} \eta_{1}(\varepsilon)=0$.

Thus $\Psi$ extends continuously and uniquely to each $[0, f]$, and hence to $\mathscr{J}$ in such a way that it is continuous on each order-interval. In particular, we must have $0 \leq \Delta_{\Psi}(f, g) \leq \Delta_{\Lambda}(f, g)$ for every $f, g \in \mathscr{I}$. Further, if $f \in \mathscr{I}$, we may pick $f_{n} \in \mathscr{V}$ so that $f_{n} \uparrow f$. Then as $\lim \sup \left|\Phi\left(f_{n}\right)-\Phi(f)\right| \leq 2 \delta\|f\|$ by Lemma 4.3 we obtain $d(\Phi, \Psi) \leq M+4 \delta$. This in turn implies that $\Psi$ is semilinear and we can apply Theorem 5.2 to show that $\Psi=\Phi_{X}$ for some Köthe function space $X$.

We remark that if $\Phi$ is continuous then we can improve the estimate to give $d\left(\Phi, \Phi_{X}\right) \leq M$.

We now prove a result on set functions which is the key to our main results. 
Theorem 6.2. Let $S$ be a finite set and let $\mu$ be a positive measure on $S$. Let $\phi: 2^{S} \rightarrow \mathbf{R}$ be a set function, continuous with respect to $\mu$ in the sense that $\mu(A)=0$ implies $\phi(A)=0$, and such that whenever $A, B$ are disjoint sets then

$$
\phi(A)+\phi(B)-\mu(A \cup B) \leq \phi(A \cup B) \leq \phi(A)+\phi(B) .
$$

Then there is a signed measure $\nu$ on $S$ such that $\nu(A) \leq \phi(A)$ for $A \subset S$ and $\nu(S) \geq \phi(S)-4 \mu(S)$.

Proof. For each $A \subset S$ let $\Gamma_{A}$ be the set of signed measures $\rho$ defined on $A$ so that $\rho \leq \phi$. Let

$$
h(A)=\mu(A)^{-1}\left(\phi(A)-\sup _{\rho \in \Gamma_{A}} \rho(A)\right) .
$$

Here we define $h(A)=0$ if $\mu(A)=0$. Then $h(S) \leq \max _{A \subset S} h(A)$. We shall show that $\max _{A \subset S} h(A) \leq 4$. Once this is done it will follow that there is a sequence $\nu_{n} \in \Gamma_{S}$ with $\nu_{n}(S) \geq \phi(S)-4 \mu(S)-1 / n$; it is then easy to show that $\left(\nu_{n}(A)\right)$ is bounded for every $A$ and so a compactness argument will complete the proof. Thus, by restricting to the set where $h$ attains its maximum, it suffices to consider the case when $h(A) \leq h(S)=\gamma$, say for every $A$.

We may clearly suppose $\mu(S)>0$. For $0<\varepsilon<1$, pick a measure $\lambda \in \Gamma_{S}$ so that $\lambda(S) \geq \phi(S)-\varepsilon-\gamma \mu(S)$. Let $E$ be a maximal subset of $S$ so that $\lambda(E) \geq \phi(E)-2 \mu(E)$. Let $F=S \backslash E$. If $F$ is empty we are done. Otherwise, if $A \subset F$ then $\lambda(A \cup E)<\phi(A \cup E)-2 \mu(A \cup E)$, so that $\lambda(A)<\phi(A \cup E)-$ $\phi(E)-2 \mu(A) \leq \phi(A)-2 \mu(A)$. Now consider the signed measure $\lambda_{0}$ where $\lambda_{0}(B)=\lambda(B)+\mu(B \cap F)-\mu(B \cap E)$. Then

$$
\begin{aligned}
\lambda_{0}(B) & \lambda(B \cap E)-\mu(B \cap E)+\lambda(B \cap F)+\mu(B \cap F) \\
& <\phi(B \cap E)-\mu(B \cap E)+\phi(B \cap F)-\mu(B \cap F) \\
& \leq \phi(B)
\end{aligned}
$$

so that $\lambda_{0} \in \Gamma_{S}$. Thus $\lambda_{0}(S) \leq \phi(S)-\gamma \mu(S)$ or $\lambda(S)+\mu(F)-\mu(E) \leq \lambda(S)+\varepsilon$. Thus $\mu(E) \geq \frac{1}{2}(1-\varepsilon) \mu(S)$.

Now we utilize the fact that $h(F) \leq \gamma$. There exists a signed measure $\nu$ on $F$ with $\nu \in \Gamma_{F}$ and $\nu(F) \geq \phi(f)-\gamma \mu(F)-\varepsilon$. Define $\nu_{0}$ on $S$ by

$$
\nu_{0}(B)=\lambda(B \cap E)+\nu(B \cap F)-\mu(B) .
$$

Then

$$
\nu_{0}(B) \leq \phi(B \cap E)+\phi(B \cap F)-\mu(B) \leq \phi(B) .
$$

Thus $\nu_{0} \in \Gamma_{S}$, and

$$
\nu_{0}(S)=\lambda(E)+\nu(F)-\mu(S) \leq \phi(S)-\lambda \mu(S) .
$$

Hence

$$
\phi(E)+\phi(F)-2 \mu(E)-\gamma \mu(F)-\varepsilon-\mu(S) \leq \phi(S)-\gamma \mu(S) .
$$

Since $\phi(E)+\phi(F) \geq \phi(S)$ this simplifies to $(\gamma-2) \mu(E) \leq \mu(S)+\varepsilon$. Thus

$$
\gamma-2 \leq \frac{2}{1-\varepsilon}+\frac{2 \varepsilon}{1-\varepsilon} \frac{1}{\mu(S)} .
$$

Letting $\varepsilon \rightarrow 0$ we obtain $\gamma \leq 4$.

In order to apply Theorem 6.2 we will need some preliminary estimates for semilinear functionals. Essentially, the next theorem is a translation of a result in [25] but we give a more precise proof. 
Theorem 6.3. Suppose $\sigma>1$. Then there are constants $C(\sigma)$ and $C^{\prime}(\sigma)$ so that whenever $\mathscr{I} \subset(L \log L)_{+}$is a strict semi-ideal and $\Phi: \mathscr{I} \rightarrow \mathbf{R}$ is a map which satisfies

$$
\Delta_{\Phi}(f, g) \leq \alpha \log 2(\|f\|+\|g\|)
$$

for $f, g \in \mathscr{I}$ (respectively, for disjoint $f, g \in \mathscr{I}$ ) then for any $f_{1}, \ldots, f_{n} \in \mathscr{I}$ (respectively, any disjoint $f_{1}, \ldots, f_{n} \in \mathscr{I}$ ) we have, if $f=\sum_{k=1}^{n} f_{k}$

$$
\begin{gathered}
\Delta_{\Phi}\left(f_{1}, \ldots, f_{n}\right) \leq \alpha\left(\sigma \sum_{k=1}^{n}\left\|f_{k}\right\| \log \frac{\|f\|}{\left\|f_{k}\right\|}+C\|f\|\right), \\
\Delta_{\Phi}\left(f_{1}, \ldots, f_{n}\right) \leq \alpha\left(\sigma \sum_{k=1}^{n}(\log k)\left\|f_{k}\right\|+C^{\prime}\|f\|\right) .
\end{gathered}
$$

Proof. By a simple continuity argument there exists $0<\theta_{0}<1$ and $\tau>0$ so that if $\frac{1}{2} \leq \alpha \leq \frac{1}{2}\left(1+\theta_{0}\right)$ then

$$
\sigma\left(a \log \frac{1}{a}+(1-a) \log \frac{1}{1-a}\right) \geq \log 2+\tau .
$$

Let $c_{n}$ be the least constant so that if $f_{1}, \ldots, f_{n} \in \mathscr{I}$ satisfy $\sum_{k=1}^{n}\left\|f_{k}\right\|=1$ (and are disjoint) then

$$
\Delta_{\Phi}\left(f_{1}, \ldots, f_{n}\right) \leq \alpha\left(\sigma \sum_{k=1}^{n}\left\|f_{k}\right\| \log \frac{1}{\left\|f_{k}\right\|}+c_{n}\right) .
$$

It is clear that for every $n, c_{n}$ is finite.

For any such $f_{1}, \ldots, f_{n}$ we let $\theta=\max _{1 \leq k \leq n}\left\|f_{k}\right\|$. Let us first assume that $\theta \leq \theta_{0}$. Then we may choose signs $\varepsilon_{k}= \pm 1$ so that $0 \leq \sum_{k=1}^{n} \varepsilon_{k}\left\|f_{k}\right\| \leq \theta$. Let $A=\left\{k: \varepsilon_{k}=1\right\}$ and $B=\left\{k: \varepsilon_{k}=-1\right\}$. Let $a=\sum_{k \in A}\left\|f_{k}\right\|$ so that $\frac{1}{2} \leq a \leq \frac{1}{2}(1+\theta)$. Let $f_{A}=\sum_{k \in A} f_{k}$ and let $f_{B}=\sum_{k \in B} f_{k}$. Then

$$
\begin{aligned}
\Delta_{\Phi}\left(f_{A}, f_{B}\right) & \leq \alpha \log 2, \\
\Delta_{\Phi}\left(f_{k}: k \in A\right) & \leq \alpha\left(\sum_{k \in A}\left\|f_{k}\right\| \log \frac{a}{\left\|f_{k}\right\|}+c_{n} a\right), \\
\Delta_{\Phi}\left(f_{k}: k \in B\right) & \leq \alpha\left(\sum_{k \in B}\left\|f_{k}\right\| \log \frac{1-a}{\left\|f_{k}\right\|}+c_{n}(1-a)\right), \\
\Delta_{\Phi}\left(f_{1}, \ldots, f_{n}\right) & =\Delta_{\Phi}\left(f_{k}: k \in A\right)+\Delta_{\Phi}\left(f_{k}: k \in B\right)+\Delta_{\Phi}\left(f_{A}, f_{B}\right) .
\end{aligned}
$$

Combining these equations gives us

$$
\begin{aligned}
& \Delta_{\Phi}\left(f_{1}, \ldots, f_{n}\right)-\alpha \sigma \sum_{k=1}^{n}\left\|f_{k}\right\| \log \frac{1}{\left\|f_{k}\right\|} \\
& \quad \leq \alpha\left(c_{n}+\sigma(a \log a+(1-a) \log (1-a))+\log 2\right) .
\end{aligned}
$$

By the hypothesis on $\theta$ we conclude that

$$
\Delta_{\Phi}\left(f_{1}, \ldots, f_{n}\right)-\alpha \sigma \sum_{k=1}^{n}\left\|f_{k}\right\| \log \frac{1}{\left\|f_{k}\right\|} \leq \alpha\left(c_{n}-\tau\right) .
$$


On the other hand, if $\theta>\theta_{0}$ we may assume $\left\|f_{n}\right\|=\theta$. Then

$$
\begin{aligned}
\Delta_{\Phi}\left(f_{1}, \ldots, f_{n}\right) & =\Delta_{\Phi}\left(\sum_{k=1}^{n} f_{k}, f_{n}\right)+\Delta_{\Phi}\left(f_{1}, \ldots, f_{n-1}\right) \\
& \leq \alpha\left(\log 2+\sigma \sum_{k=1}^{n-1}\left\|f_{k}\right\| \log \frac{1-\theta}{\left\|f_{k}\right\|}+c_{n}(1-\theta)\right) \\
& \leq \alpha\left(\log 2+\sigma \sum_{k=1}^{n}\left\|f_{k}\right\| \log \frac{1}{\left\|f_{k}\right\|}+c_{n}(1-\theta)\right)
\end{aligned}
$$

so that

$$
\Delta_{\Phi}\left(f_{1}, \ldots, f_{n}\right)-\sigma \alpha \sum_{k=1}^{n}\left\|f_{k}\right\| \log \frac{1}{\left\|f_{k}\right\|} \leq \alpha\left(\log 2+c_{n}\left(1-\theta_{0}\right)\right) .
$$

Combining the two cases we must have that $c_{n} \leq \log 2+c_{n}\left(1-\theta_{0}\right)$ or $c_{n} \leq$ $\log 2 / \theta_{0}$ independent of $n$. This leads to the first equation. The second is obtained by using Lemma 3.5 of [25], observing that it first suffices to consider the case $\left\|f_{1}\right\| \geq\left\|f_{2}\right\| \geq \cdots \geq\left\|f_{n}\right\|$.

Lemma 6.4. Given $\varepsilon>0$ there exists a constant $\beta=\beta(\varepsilon)<\infty$ so that whenever $\mathscr{I}$ is a semi-ideal and $\Phi$ is a real semilinear functional on $\mathscr{I}$ and $f_{1}, \ldots, f_{n} \in$

$$
\left|\Delta_{\Phi}\left(f_{1}, \ldots, f_{n}\right)\right| \leq \delta(\Phi)\left(\varepsilon \sum_{k=1}^{n} k\left\|f_{k}\right\|+\beta\left\|\sum_{k=1}^{n} f_{k}\right\|\right)
$$

Proof. We have

$$
\left|\Delta_{\Phi}\left(f_{1}, \ldots, f_{n}\right)\right| \leq 2 \delta \sum_{k=1}^{n}(\log k)\left\|f_{k}\right\|+C \sum_{k=1}^{n}\left\|f_{k}\right\|
$$

for a suitable constant $C$, by applying Theorem 6.3 with $\sigma=2 \log 2>1$. Since for arbitrary $\varepsilon$ there exists $\gamma=\gamma(\varepsilon)$ so that $\log k \leq \varepsilon k+\gamma$ for all $k$ the lemma follows easily.

Lemma 6.5. Given $\varepsilon>0$ there is a constant $C(\varepsilon)$ so that if $\mathscr{J}$ is a strict semiideal and $\Phi$ is a real semilinear functional on $\mathscr{I}$ such that for any disjoint $f, g$ we have $\Delta_{\Phi}(f, g) \geq 0$, then for any $f_{1}, \ldots, f_{n} \in \mathscr{I}$,

$$
\Delta_{\Phi}\left(f_{1}, \ldots, f_{n}\right)+\delta \varepsilon \Delta_{\Lambda}\left(f_{1}, \ldots, f_{n}\right) \geq-C \delta \sum_{k=1}^{n}\left\|f_{k}\right\|,
$$

where $\delta=\delta(\Phi)$.

Proof. We first note that we need only establish the lemma for simple functions. Indeed, if we establish the lemma for simple functions then it follows for arbitrary functions by using Lemma 4.3 with $C$ replaced by $C+4$. Assume then that $f_{1}, \ldots, f_{n}$ are simple. We may further assume that if $f=f_{1}+\cdots+f_{n}$ then $\|f\|=1$. Let $\Sigma_{0}$ be the finite algebra generated by $f_{1}, \ldots, f_{n}$.

If we set $\phi(A)=\Phi\left(f 1_{A}\right)$ then $\phi$ is a set function on $\Sigma_{0}$ which satisfies for disjoint sets $A, B$

$$
\phi(A)+\phi(B)-\delta \int_{A \cup B} f d \mu \leq \phi(A \cup B) \leq \phi(A)+\phi(B) .
$$


Thus Theorem 6.3 can be applied to produce a signed measure $\nu$ on $\Sigma_{0}$ with $\nu(A) \leq \phi(A)$ for all $A \in \Sigma_{0}$ and $\nu(S) \geq \phi(S)-4 \delta$.

Now suppose $g$ is any $\Sigma_{0}$-measurable function whose support is contained in that of $f$. Suppose $\|g\|=1$ and let $h=g / f(h=0$ when $f=0)$. Let $A_{0}=\{h<1\}$ and then for $k>0$ set $A_{k}=\left\{2^{k-1} \leq h<2^{k}\right\}$. There exists $N$ so that $A_{k}=\varnothing$ for $k>N$. We first notice that

$$
\Delta_{\Phi}\left(g 1_{A_{0}}, g 1_{A_{1}}, \ldots, g 1_{A_{N}}\right) \leq \delta\left(\varepsilon \log 2 \sum_{k=0}^{N}(k+1)\left\|g 1_{A_{k}}\right\|+C_{0}\right)
$$

by Lemma 6.4 , where $C_{0}$ depends only on $\varepsilon$. Now

$$
\log 2 \sum_{k=0}^{N}(k+1) \int_{A_{k}} g d \mu \leq \int g\left(\log _{+} h+2 \log 2\right) d \mu .
$$

Further

$$
\begin{aligned}
\int g \log _{+} h d \mu & \leq \int g \log h d \mu+\int_{A_{0}} g \log \frac{f}{g} d \mu \\
& \leq \int g \log h d \mu+\frac{1}{e} \int_{A_{0}} f d \mu \\
& \leq \int g \log h d \mu+\frac{1}{e} .
\end{aligned}
$$

Combining these equations gives us

$$
\Delta_{\Phi}\left(g 1_{A_{0}}, \ldots, g 1_{A_{N}}\right) \leq \delta\left(\varepsilon \int g \log h d \mu+C_{1}\right),
$$

where $C_{1}$ depends only on $\varepsilon$. Now we can write each $h 1_{A_{k}}$ in the form

$$
h 1_{A_{k}}=2^{k} \sum_{j=1}^{\infty} 2^{-j} 1_{B_{k j}}
$$

where $B_{k j} \in \Sigma_{0}$. Now for arbitrary $m$ we have

$$
\begin{aligned}
\Delta_{k m} & =\Delta_{\Phi}\left(2^{-1} f 1_{B_{k 1}}, \ldots, 2^{-m} f 1_{B_{k m}}\right) \\
& \leq \delta\left(\sum_{j=1}^{m} j 2^{-j}\left\|f 1_{B_{k j}}\right\|+C_{2} \sum_{j=1}^{m} 2^{-j}\left\|f 1_{B_{k} j}\right\|\right),
\end{aligned}
$$

where $C_{2}$ is a constant. Thus $\Delta_{k m} \leq C_{3} \delta\left\|f 1_{A_{k}}\right\|$ for some constant $C_{3}$. Now this means that

$$
\Phi\left(\sum_{j=1}^{m} 2^{k-j} f 1_{B_{k j}}\right) \geq \sum_{j=1}^{m} 2^{k-j} \phi\left(B_{k j}\right)-C_{3} \delta 2^{k}\left\|f 1_{A_{k}}\right\| .
$$

Thus for $k \geq 1$,

$$
\Phi\left(\sum_{j=1}^{m} 2^{k-j} f 1_{B_{k j}}\right) \geq \sum_{j=1}^{m} 2^{k-j} \nu\left(B_{k j}\right)-2 C_{3} \delta\left\|g 1_{A_{k}}\right\| .
$$


Letting $m \rightarrow \infty$ and invoking Lemma 4.3 we have

$$
\Phi\left(g 1_{A_{k}}\right) \geq \int_{A_{k}} h d \nu-C_{4} \delta \int_{A_{k}} g d \mu,
$$

where $C_{4}$ is a constant. In the case $k=0$ the same reasoning leads to

$$
\Phi\left(g 1_{A_{0}}\right) \geq \int_{A_{0}} h d \nu-C_{4} \delta \int_{A_{0}} f d \mu .
$$

Combining we obtain $\sum_{k=0}^{N} \Phi\left(g 1_{A_{k}}\right) \geq \int h d \nu-2 C_{4} \delta$. Thus

$$
\Phi(g) \geq \int h d \nu-\varepsilon \delta \int g \log h d \mu-C_{5} \delta,
$$

where $C_{5}=C_{5}(\varepsilon)$. Now we apply this to $g_{k}=f_{k} /\left\|f_{k}\right\|$. The result is that

$$
\Phi\left(f_{k}\right) \geq \int \frac{f_{k}}{f} d \nu-\varepsilon \delta \int f_{k} \log \frac{f_{k}}{f\left\|f_{k}\right\|} d \mu-C_{5} \delta\left\|f_{k}\right\| .
$$

Summing over $k$

$$
\sum_{k=1}^{n} \Phi\left(f_{k}\right) \geq \nu(S)-\varepsilon \delta \Delta_{\Lambda}\left(f_{1}, \ldots, f_{n}\right)-C_{5} \delta
$$

Recalling the choice of $\nu$ this implies

$$
\Delta_{\Phi}\left(f_{1}, \ldots, f_{n}\right)+\varepsilon \delta \Delta_{\Lambda}\left(f_{1}, \ldots, f_{n}\right) \geq-\left(C_{5}+4\right) \delta .
$$

We finally come to the main theorem of the section.

Theorem 6.6. Given $\varepsilon>0$ there is a constant $C=C(\varepsilon)$ so that whenever $\mathscr{I}$ is a strict semi-ideal contained in $(L \log L)_{+}$and $\Phi$ is a real semilinear functional with $\delta(\Phi) \leq(1-\varepsilon) \log 2$ then there is a Köthe function space $X$ with $d\left(\Phi, \Phi_{X}-\Phi_{X^{*}}\right) \leq C$.

Proof. By Theorem 6.3 we have that if $f_{1}, \ldots, f_{n}$ are disjoint and $f=f_{1}+$ $\cdots+f_{n}$ then

$$
\left|\Delta_{\Phi}\left(f_{1}, \ldots, f_{n}\right)\right| \leq\left(1-\frac{1}{2} \varepsilon\right) \sum_{k=1}^{n}\left\|f_{k}\right\| \log \frac{\|f\|}{\left\|f_{k}\right\|}+C_{0}\|f\|,
$$

where $C_{0}$ depends only on $\varepsilon$. But this can be reworded as

$$
\left|\Delta_{\Phi}\left(f_{1}, \ldots, f_{n}\right)\right| \leq\left(1-\frac{1}{2} \varepsilon\right) \Delta_{\Lambda}\left(f_{1}, \ldots, f_{n}\right)+C_{0}\|f\| .
$$

Let us define $\Psi_{0}=\Phi+\left(1-\frac{1}{2} \varepsilon\right) \Lambda$. We clearly then have for disjoint $f_{1}, \ldots, f_{n}$

$$
\Delta_{\Psi_{0}}\left(f_{1}, \ldots, f_{n}\right) \geq-C_{0} \sum_{k=1}^{n}\left\|f_{k}\right\|
$$

and $\delta\left(\Psi_{0}\right) \leq 2 \log 2$. Let $\Psi(f)=\inf \sum_{k=1}^{n} \Psi_{0}\left(f_{k}\right)$ where the infimum is taken over all disjoint $f_{1}, \ldots, f_{n}$ with $f=\sum f_{k}$. Clearly $\Psi_{0}(f)-C_{0}\|f\| \leq \Psi(f) \leq$ $\Psi_{0}(f)$ and hence for any $f_{1}, \ldots, f_{n}$

$$
\left|\Delta_{\Psi}\left(f_{1}, \ldots, f_{n}\right)-\Delta_{\Psi_{0}}\left(f_{1}, \ldots, f_{n}\right)\right| \leq C_{0} \sum_{k=1}^{n}\left\|f_{k}\right\| .
$$


Also $\delta(\Psi) \leq 2 \log 2+C_{0}$. Now $\Delta_{\Psi}(f, g) \geq 0$ for disjoint $f, g$ so that there is a constant $C_{1}=C_{1}(\varepsilon)$ from Lemma 6.5 with

$$
\Delta_{\Psi}\left(f_{1}, \ldots, f_{n}\right) \geq-\frac{\varepsilon}{2} \Delta_{\Lambda}\left(f_{1}, \ldots, f_{n}\right)-C_{1} \sum_{k=1}^{n}\left\|f_{k}\right\|
$$

for arbitrary $f_{1}, \ldots, f_{n}$. But this means that

$$
\Delta_{\Psi_{0}}\left(f_{1}, \ldots, f_{n}\right) \geq \frac{\varepsilon}{2} \Delta_{\Lambda}\left(f_{1}, \ldots, f_{n}\right)-C_{2} \sum_{k=1}^{n}\left\|f_{k}\right\| .
$$

Hence

$$
\Delta_{\Phi}\left(f_{1}, \ldots, f_{n}\right) \geq-\Delta_{\Lambda}\left(f_{1}, \ldots, f_{n}\right)-C_{2} \sum_{k=1}^{n}\left\|f_{k}\right\| .
$$

Clearly this reasoning may also be applied to $-\Phi$ and so we conclude that

$$
\left|\Delta_{\Phi}\left(f_{1}, \ldots, f_{n}\right)\right| \leq \Delta_{\Lambda}\left(f_{1}, \ldots, f_{n}\right)+C_{2} \sum_{k=1}^{n}\left\|f_{k}\right\| .
$$

Now we can apply Proposition 6.1 to $\frac{1}{2}(\Phi+\Lambda)$ to deduce that there is a Köthe function space $X$ with $d\left(\frac{1}{2}(\Phi+\Lambda), \Phi_{X}\right) \leq C_{3}$ where $C_{3}$ depends only on $\varepsilon$. Hence $d\left(\Phi, \Phi_{X}-\Phi_{X^{*}}\right) \leq 2 C_{3} \quad \square$.

We now state an extension to maps defined on ideals rather than semi-ideals. Let $\mathcal{J}$ be an ideal contained in $L \log L$. Then a homogeneous map $\Phi: \mathscr{J} \rightarrow \mathbf{C}$ will be called semilinear if $\left.\Phi\right|_{\mathcal{J}+}$ is semilinear and (4.5) holds for all $f, g \in \mathcal{J}$. $\Phi$ is called real if its restriction to $\mathscr{J}_{+}$is real.

Corollary 6.7. Suppose $\varepsilon>0$; then there exists a constant $C=C(\varepsilon)$ so that if $\mathcal{J}$ be a strict ideal contained in $L_{1}$ and if $\Phi: \mathcal{J} \rightarrow \mathbf{C}$ be a real semilinear map with $\delta(\Phi)<(1-\varepsilon) \log 2$ then there exists a Köthe function space $X$ so that

$$
\left|\Phi(f)-\left(\Phi_{X}(f)-\Phi_{X^{*}}(f)\right)\right| \leq C\|f\|
$$

for all $f \in \mathcal{J}$. If $\Phi$ is not necessarily real, then we can conclude that there exist Köthe function spaces $X, Y$ so that

$$
\left|\Phi(f)-\left(\Phi_{X}(f)+i \Phi_{Y}(f)-\Phi_{X^{*}}(f)-i \Phi_{Y^{*}}(f)\right)\right| \leq C\|f\| .
$$

Proof. We apply Theorem 6.6, when $\Phi$ is real, to approximate $\left.\Phi\right|_{\mathscr{f}_{+}}$and then notice that for arbitrary $f$ we can write $f=(\mathfrak{R} f)_{+}-(\mathfrak{R} f)_{-}+i(\mathfrak{I} f)_{+}-i(\mathfrak{I} f)_{-}=$ $f_{1}-f_{2}+i f_{3}-i f_{4}$, say. Thus

$$
\left|\Phi(f)-\Phi\left(f_{1}\right)+\Phi\left(f_{2}\right)-i \Phi\left(f_{3}\right)+i \Phi\left(f_{4}\right)\right| \leq C_{0}\|f\|
$$

for some constant $C$. A similar inequality holds for $\Phi_{X}$ and $\Phi_{X^{*}}$ by Lemma 5.6 and so the result follows. If $\Phi$ is complex, one uses the same argument on its real and imaginary parts when restricted to $\mathscr{J}_{+}$.

Let us give an application to minimal extensions (see $\S 1)$. Compare the original constructions of minimal extensions of $l_{1}$ in $[23,44]$.

Theorem 6.8. Let $Z$ be a minimal extension of the real space $L_{1, \mathbf{R}}(S, \Sigma, \mu)$. Then there exists a Köthe function space $X$ so that $Z$ is linearly homeomorphic to the completion of $L_{2}(\mu) \oplus \mathbf{R}$ under the quasinorm

$$
\|(f, \alpha)\|=\|f\|+\left|\alpha-\Phi_{X}(f)+\Phi_{X^{*}}(f)\right| \text {. }
$$


Proof. In fact there is a quasilinear map $F: L_{1} \rightarrow \mathbf{R}$ so that $Z$ can be identified with the space $L_{1} \oplus_{F} \mathbf{R}$ of all pairs $(f, \alpha)$ quasinormed by $\|(f, \alpha)\|=\|f\|+$ $|\alpha-F(f)|$. Now consider $F$ restricted to $E$, where $E$ is a suitable weighted $L_{2}$-space so that $\|x\|_{E} \geq\|x\|$ for every $x \in L_{0}$. Since $L_{2}$ is a $\mathscr{K}$-space [23], there exists a linear map (not necessarily continuous!) $\phi: L_{2} \rightarrow \mathbf{R}$ with $|F(f)-\phi(f)| \leq C\|f\|_{E}$ for some constant $C$. Now $F-\phi$ will be semilinear according to our definition on the ideal $E$. Thus there exists a Köthe function space $X$ and $\alpha>0$ so that

$$
\left|F(f)-\phi(f)-\alpha\left(\Phi_{X}(f)-\Phi_{X^{*}}(f)\right)\right| \leq C_{1}\|f\|
$$

for $f \in E$ by the real version of Corollary 6.7. Now this implies [23, Theorem 2.5] the isomorphism of the space $Z$ with the minimal extension generated by $\Phi_{X}-\Phi_{X^{*}}$.

\section{Applications to Centralizers}

Suppose $X$ is a Köthe function space and $\Omega: X \rightarrow L_{0}$ is a centralizer on $X$. We say $\Omega$ is real if $\Omega(f)$ is a real function whenever $f$ is a real function.

Lemma 7.1. Let $\Omega$ be a centralizer on $X$ with $\rho(\Omega)=\rho$. Then there exist real centralizers $\Omega_{1}, \Omega_{2}$ so that $\rho\left(\Omega_{j}\right) \leq 2 \rho$ for $j=1,2$ and

$$
\left\|\Omega(f)-\Omega_{1}(f)-i \Omega_{2}(f)\right\|_{X} \leq 2 \rho\|f\|_{X} \quad \text { for every } f \in X .
$$

Proof. Set $\Omega_{1}(f)=\mathfrak{R} \Omega(\mathfrak{R} f)+i \mathfrak{R} \Omega(\mathfrak{I} f)$ and $\Omega_{2}(f)=\mathfrak{I} \Omega(\mathfrak{R} f)-i \mathfrak{I}(\Omega(\mathfrak{I} f))$. The lemma then follows quickly.

We now use [29, Theorem 5.1]. If $\Omega: X \rightarrow L_{0}$ is a centralizer then there is a centralizer $\Omega^{[1]}$ on $L_{1}$ with $\rho\left(\Omega^{[1]}\right) \leq 36 \rho(\Omega)$ and so that for $x \in X$ and $y \in X^{*}$ we have

$$
\left\|\Omega^{[1]}(x y)-\Omega(x) y\right\| \leq 18 \rho(\Omega)\|x\|_{X}\|y\|_{X^{*}} .
$$

Furthermore $\Omega^{[1]}$ is unique up to equivalence, i.e., if $\Omega^{\prime}$ is any other centralizer on $L_{1}$ satisfying

$$
\left\|\Omega^{\prime}(x y)-\Omega(x) y\right\| \leq C\|x\|_{X}\|y\|_{X^{*}},
$$

then for some constant $C_{1}$ we have $\left\|\Omega^{[1]}(f)-\Omega^{\prime}(f)\right\| \leq C_{1}\|f\|$ for all $f \in$ $L_{1} . \Omega^{[1]}$ is defined by $\Omega^{[1]}(f)=\Omega(x) y$, where $f=x y$ is the Lozanovskii factorization of $f$ for $X$. Clearly if $\Omega$ is real the so is $\Omega^{[1]}$.

Theorem 7.2. (i) Let $X_{0}, X_{1}$ be Köthe function spaces and let $X=\left[X_{0}, X_{1}\right]_{\theta}$. Let $\Omega$ be the induced centralizer on $X$. Then for $f \in L_{1}$,

$$
\Omega^{[1]}(f)=f\left(\log x_{1}-\log x_{0}\right),
$$

where $|f|=x_{0} y_{0}=x_{1} y_{1}$ are the Lozanovskii factorizations for $|f|$ with respect to $X_{0}, X_{1}$.

(ii) Let $\mathscr{X}=\left\{X_{w}: w \in \mathbf{T}\right\}$ be a strongly admissible family of Köthe function spaces, and $X=X_{0}$ and $\Omega$ is the centralizer induced on $X$. Then for $f \in L_{1}$

$$
\Omega^{[1]}(f)(s)=f(s)\left(\int_{-\pi}^{\pi} e^{-i t} \log g(s, t) \frac{d t}{\pi}\right),
$$

where $f(s)=g(s, t) h(s, t)$ is the Lozanovskii factorization for the space $\mathscr{E}$ on $S \times \mathbf{T}$ defined by

$$
\|\phi\|_{\mathscr{E}}=\operatorname{ess} \sup \|\phi(\cdot, w)\|_{X_{w}} .
$$


Proof. (i) Assume $f$ has norm one and is factored as stated. On $\mathscr{S}=\{z: 0<$ $\mathfrak{R} z<1\}$ consider the functions $G(z)=x_{0}^{1-z} x_{1}^{z}$ and $H(z)=\left|y_{0}\right|^{1-z}\left|y_{1}\right|^{z}$. Then both $G, H \in \mathcal{N}^{+}(\mathscr{S})$. Also $\|G(\theta)\|_{X} \leq 1$ and $\|H(\theta)\|_{X^{*}} \leq 1$ since $X^{*}=\left[X_{0}^{*}, X_{1}^{*}\right]_{\theta}$. However $G(z) H(z)=|f|$ from which it follows that $|f|=$ $G(\theta) H(\theta)$ is the Lozanovskii factorization for $X$. Now consider any optimal factorization $x=u_{0}^{1-\theta} u_{1}^{\theta}$ with $u_{0}, u_{1} \geq 0$ and $\left\|u_{0}\right\|_{X_{0}}=\left\|u_{1}\right\|_{X_{1}}=1$. Let $K(z)=u_{0}^{1-z} u_{1}^{z}$; then $\|K(z) H(z)\| \leq 1$ but $\int K(\theta) H(\theta) d \mu=1$. Hence $\int K(z) H(z) d \mu=1$ for all $z$ and so $K(z, s) H(z, s)$ is real and positive for all $z$ for almost every $s \in S$. (An argument with a dense countable set in $\mathscr{S}$ is required here.) Thus $K(z, s) H(z, s)=f(s)$ a.e. and so $u_{0}(s)=x_{0}(s)$, $u_{1}(s)=x_{1}(s)$ for $s \in \operatorname{supp} f=\operatorname{supp} x$. Now by (3.2) this implies that $\Omega(x)$ can only take the value $x\left(\log x_{1}-\log x_{0}\right)$.

Now

$$
\Omega^{[1]}(f)=\Omega(x) H(\theta) \operatorname{sgn} f,
$$

where $\operatorname{sgn} f=f /|f|$ as long as $f \neq 0$ and is zero otherwise. Hence

$$
\Omega^{[1]}(f)=f\left(\log x_{1}-\log x_{0}\right) .
$$

(ii) The proof is very similar. Suppose as above that $f \in L_{1}$ and that $|f(s)|=g(s, t) h(s, t)$ is the Lozanovskii factorization of $|f|$ for $\mathscr{E}$. Define $G, H \in \mathscr{N}^{+}$by

$$
\begin{aligned}
& G(z)=\exp \left(\int_{-\pi}^{\pi} \frac{e^{i t}+z}{e^{i t}-z} \log g(s, t) \frac{d t}{2 \pi}\right), \\
& H(z)=\exp \left(\int_{-\pi}^{\pi} \frac{e^{i t}+z}{e^{i t}-z} \log h(s, t) \frac{d t}{2 \pi}\right) .
\end{aligned}
$$

It follows from the duality theorem Corollary 4.9 that $|f|=G(0) H(0)$ is the Lozanovskii factorization for $X$. Further, arguing as in the case (i) it can be shown that if $x=G(0)$ and if $K \in \mathscr{N}^{+}$satisfies $K(0)=x$ and $\|K(w)\|_{X_{w}} \leq 1$ for $w \in \mathbf{T}$ then $K(z, s)=G(z, s)$ on supp $f$. Thus applying Theorem 3.3 we obtain the theorem.

Notice in the above theorem that in case (i) the centralizer $\Omega^{[1]}$ is independent of $\theta$. We now connect our results with those of the preceding sections. If $\Omega$ is a centralizer on $X$ we defined a strict ideal $\mathscr{J}=\mathscr{J}_{\Omega} \subset L_{1}$ by $f \in \mathcal{J}$ if and only if $\Omega^{[1]}(f) \in L_{1}$. We omit the simple proof that $\mathscr{J}$ is a strict ideal. On $\mathscr{J}$ we define the functional $\Phi^{\Omega}$ by $\Phi^{\Omega}(f)=\int \Omega^{[1]}(f) d \mu$.

Lemma 7.3. $\Phi^{\Omega}$ is a semilinear functional on $\mathcal{J}$ with $\delta\left(\Phi^{\Omega}\right) \leq 108 \rho(\Omega)$. $\Phi^{\Omega}$ is real whenever $\Omega$ is real.

Proof. Condition (4.3) is obvious and (4.4) follows from Lemma 4.2 of [29]. (4.5) follows from Lemma 4.3 of [29].

Proposition 7.4. (i) Let $X_{0}, X_{1}$ be a pair of Köthe function spaces and let $X=$ $\left[X_{0}, X_{1}\right]_{\theta}$ and suppose $\Omega$ is the corresponding centralizer on $X$. Then $\mathscr{J}_{X_{0}} \cap$ $\mathscr{I}_{X_{1}} \subset \mathscr{J}_{\Omega}$ and

$$
\Phi^{\Omega}(f)=\Phi_{X_{1}}(f)-\Phi_{X_{0}}(f), \quad f \in \mathscr{I}_{X_{0}} \cap \mathscr{J}_{X_{1}} .
$$

(ii) If $\mathscr{X}=\left\{X_{w}: w \in \mathbf{T}\right\}$ is a strongly admissible family of Köthe function spaces, and $X=X_{0}$ with $\Omega$ the corresponding centralizer, then there is strict 
semi-ideal $\mathscr{I} \subset \mathscr{I}_{\Omega}$ so that

$$
\Phi^{\Omega}(f)=\int_{-\pi}^{\pi} e^{-i t} \Phi_{X_{e^{i t}}}(f) \frac{d t}{2 \pi}, \quad f \in \mathscr{I} .
$$

The proofs of these statements are immediate.

We now prove our main theorem. We recall that two centralizers $\Omega_{1}$ and $\Omega_{2}$ on $X$ are called equivalent if there exists a constant $B$ so that $\left\|\Omega_{1}(f)-\Omega_{2}(f)\right\|_{X} \leq B\|f\|_{X}$ for all $f \in X$.

Theorem 7.6. There is a constant $\gamma>0$ and a constant $C<\infty$ so that whenever $1<p \leq 2$ and $X$ is a p-convex and q-concave Köthe function space with $\frac{1}{p}+\frac{1}{q}=1$ and $\Omega$ is a real centralizer on $X$ with $\rho(\Omega) \leq \gamma / q$ then there is $a$ pair of Köthe function spaces $X_{0}, X_{1}$ so that $X=\left[X_{0}, X_{1}\right]_{1 / 2}$ and if $\Omega_{0}$ is the induced centralizer then $\left\|\Omega(f)-\Omega_{0}(f)\right\|_{X} \leq C\|f\|_{X}$ for $f \in X$. In particular $\Omega$ is equivalent to $\Omega_{0}$.

Furthermore $X_{0}$ and $X_{1}$ are unique up to equivalent norming. If $Y_{0}, Y_{1}$ are Köthe function spaces such that $\left[Y_{0}, Y_{1}\right]_{1 / 2}=X$ with an equivalent norm and the induced centralizer $\Omega_{1}$ is equivalent to $\Omega$ then $Y_{0}=X_{0}$ with an equivalent norm and $Y_{1}=X_{1}$ with an equivalent norm.

Proof. Let us dispose of the uniqueness question. If $\Omega_{1}$ is equivalent to $\Omega$ then $\Omega_{1}^{[1]}$ and $\Omega^{[1]}$ are equivalent. Hence on a suitable strict semi-ideal, $\Phi^{\Omega}$ is equivalent to $\Phi_{Y_{1}}-\Phi_{Y_{0}}$, while $\Phi_{Y_{1}}+\Phi_{Y_{0}}$ is equivalent to $2 \Phi_{X}$. Thus, up to equivalence $\Phi_{Y_{1}}$ and $\Phi_{Y_{0}}$ are uniquely determined. Proposition 4.5 shows then that the spaces $Y_{0}, Y_{1}$, if they exist, are unique up to equivalence of norm.

The above argument quickly modifies to establish existence of $X_{0}, X_{1}$. Indeed we will take $\gamma=\frac{1}{200}$. Then $\rho\left(\Omega^{[1]}\right) \leq 0.18 q^{-1}$ and hence by Lemma 4.3 of [29], $\delta\left(\Phi^{\Omega}\right) \leq 0.54 q^{-1}$. Thus we can find a Köthe function space $W$ so that if $f \in \mathcal{J}=L \log L \cap \mathscr{J}_{\Omega}$ then

$$
\left|q \Phi^{\Omega}(f)-\left(\Phi_{W}(f)-\Phi_{W^{*}}(f)\right)\right| \leq C_{0}\|f\|,
$$

where $C_{0}$ is a universal constant (Corollary 6.7). Now on $\mathscr{J}_{+}$we consider $\Phi_{0}=\Phi_{X}-\frac{1}{q}\left(\Phi_{W}-\Phi_{W^{*}}\right)$ and $\Phi_{1}=\Phi_{X}+\frac{1}{q}\left(\Phi_{W}-\Phi_{W^{*}}\right)$. If $f, g \in \mathcal{J}_{+}$then

$$
\begin{aligned}
\Delta_{\boldsymbol{\Phi}_{0}}(f, g) & \geq \Delta_{\Phi_{X}}(f, g)-\frac{1}{q} \Delta_{\boldsymbol{\Phi}_{W}}(f, g) \\
& \geq \Delta_{\boldsymbol{\Phi}_{X}}(f, g)-\frac{1}{q} \Delta_{\Lambda}(f, g) \geq 0
\end{aligned}
$$

since $X$ is $q$-concave (Theorem 5.3). Similarly,

$$
\begin{aligned}
\Delta_{\Phi_{0}}(f, g) & \leq \Delta_{\Phi_{X}}(f, g)+\frac{1}{q} \Delta_{\Phi_{W^{*}}}(f, g) \\
& \leq \Delta_{\Phi_{X}}(f, g)+\left(1-\frac{1}{p}\right) \Delta_{\Lambda}(f, g) \\
& \leq \Delta_{\Lambda}(f, g)
\end{aligned}
$$

since $X$ is $p$-convex, again utilizing Theorem 5.3. Thus by Theorem 5.2 there is a Köthe function space $X_{0}$ so that on $\mathscr{J}_{+}$we have $\Phi_{0}=\Phi_{X_{0}}$. A similar calculation shows that $\Phi_{1}=\Phi_{X_{1}}$ for some Köthe function space $X_{1}$. Since $\Phi_{X_{0}}+\Phi_{X_{1}}=2 \Phi_{X}$ on $\mathscr{J}_{+}$we have $\left[X_{0}, X_{1}\right]_{1 / 2}=X$, by Proposition 4.5 . Let 
$\Omega_{0}$ be the corresponding induced centralizer. Then $\Phi^{\Omega_{0}}=\frac{1}{q}\left(\Phi_{W}-\Phi_{W^{*}}\right)$ on $\mathscr{J}_{+}$. Thus for $f \in \mathscr{J}_{+},\left|\Phi^{\Omega}(f)-\Phi^{\Omega_{0}}(f)\right| \leq C_{0} q^{-1}\|f\|$.

Now $\delta\left(\Phi^{\Omega}\right) \leq 0.54 q^{-1}$ and $\delta\left(\Phi^{\Omega_{0}}\right) \leq q^{-1} \log 2$. Hence, by breaking up an arbitrary $f \in \mathscr{J}$ into its real and imaginary and then positive and negative parts as in Theorem 6.7 , it follows that for all $f \in \mathcal{J}$ we have $\left|\Phi^{\Omega}(f)-\Phi^{\Omega_{0}}(f)\right| \leq$ $C_{1} q^{-1}\|f\|$ for some universal constant $C_{1}$.

Now suppose $x \in X$ and let $y \in X^{*}$ be such that $x y \in \mathcal{J}$. Then $\Omega^{[1]}(x y)$, $\Omega_{0}^{[1]}(x y) \in L_{1}$ and hence both $\Omega(x) y$ and $\Omega_{0}(x) y$ are integrable. Further, it follows from Theorem 2.6 that $\rho\left(\Omega_{0}\right)$ is bounded by a universal constant $C_{2}$. Thus,

$$
\begin{aligned}
\left|\int\left(\Omega(x)-\Omega_{0}(x)\right) y d \mu\right| \leq & \left|\int \Omega^{[1]}(x y)-\Omega_{0}^{[1]}(x y) d \mu\right| \\
& +18\left(\rho(\Omega)+\rho\left(\Omega_{0}\right)\right)\|x\|_{X}\|y\|_{X^{*}} \\
\leq & \left|\Phi^{\Omega}(x y)-\Phi^{\Omega_{0}}(x y)\right|+C_{3}\|x\|_{X}\|y\|_{X^{*}} \\
\leq & C_{4}\|x\|_{X}\|y\|_{X^{*}},
\end{aligned}
$$

where $C_{3}, C_{4}$ are universal constants. By considering all such $y$ we obtain

$$
\left\|\Omega(x)-\Omega_{0}(x)\right\|_{X} \leq C_{4}\|x\|_{X}
$$

and the proof is complete.

Remark. In order that $X$ can be renormed to be $p$-convex and $q$-concave where $q<\infty$, it is necessary and sufficient that $X$ is super-reflexive. Thus Theorem 7.6 holds for super-reflexive $X$ but not necessarily isometrically.

We recall that if $X$ is a rearrangement-invariant Banach function space on $S$ then a centralizer $\Omega$ is called symmetric if for some constant $C$ we have $\|\Omega(x)-\Omega(x \circ \sigma)\|_{X} \leq C\|x\|_{X}$ for all $x \in X$ and all measure-preserving automorphisms $\sigma$ of $S$.

Corollary 7.7. If $\mu$ is nonatomic or $S=\mathbf{N}$ with $\mu$ counting measure, and the space $X$ in Theorem 7.6 is rearrangement invariant and $\Omega$ is a symmetric centralizer then the spaces $X_{0}, X_{1}$ can be chosen to be rearrangement invariant.

Proof. In the proof of Theorem 7.6 observe that if follows from the equivalence of $q^{-1} \Phi^{\Omega}$ and $2 \Phi_{W}-\Phi_{\Lambda}$ on $\mathscr{J}_{+}$that for some constant $C_{1}$ one has $\mid \Phi_{W}(f)-$ $\Phi_{W}(f \circ \sigma) \mid \leq C_{1}\|f\|$ for every measure-preserving automorphism. From this it is easy to deduce that if $x \in B_{W}$ then $e^{-C_{1}} \leq\|x \circ \sigma\|_{W} \leq e^{C_{1}}$ so that $W$ can be renormed to be rearrangement invariant. Continuing with the proof then yields that $X_{0}, X_{1}$ are also rearrangement invariant.

We now use Corollary 7.7 to prove [29, Theorem 6.10 ] under the additional hypothesis of super-reflexivity.

Corollary 7.8. Suppose either $\mu$ is nonatomic or $S=\mathbf{N}$ with counting measure. Suppose $1<p_{0}<p_{1}<\infty$, and that $T$ is an operator of strong types $\left(p_{0}, p_{0}\right)$ and $\left(p_{1}, p_{1}\right)$. Let $X$ be any super-reflexive rearrangement-invariant Banach function space whose Boyd indices satisfy $p_{0}<p_{X}<q_{X}<p_{1}$. Then for any symmetric centralizer $\Omega$ on $X$ there is a constant $C$ so that $\|[T, \Omega](x)\|_{X} \leq$ $C\|x\|_{X}$.

Proof. We may suppose $X$ is $p$-convex and $q$-concave where $\frac{1}{p}+\frac{1}{q}=1$. We may further simplify by noting that it is only necessary by Lemma 7.1 to 
consider the case when $\Omega$ is real and $\rho(\Omega)$ is sufficiently small. But then there are r.i. spaces $X_{0}, X_{1}$ so that $X=\left[X_{0}, X_{1}\right]_{1 / 2}$ and the induced centralizer is equivalent to $\Omega$. Now by interpolation there exists $\varepsilon>0$ so that if $\theta=\frac{1}{2} \pm \varepsilon$, then $p_{0}<p_{X_{\theta}}<q_{X_{\theta}}<p_{1}$. Hence $T$ is bounded on $X_{1 / 2 \pm \varepsilon}$ and Theorem 2.6 will yield the result.

The above theorem works for real centralizers. For the general case it is possible to realize an arbitrary centralizer as a derivation induced by a family of three spaces. We have:

Theorem 7.9. There is a constant $\gamma>0$ so that if $X$ is a Köthe function space which is p-convex and q-concave where $\frac{1}{p}+\frac{1}{q}=1$ and $1<p \leq 2$ and $\Omega$ is a centralizer on $X$ with $\rho(\Omega) \leq \gamma q^{-1}$ then there are three Köthe function spaces $X(j): j=1,2,3$ so that if we consider the family $\mathscr{Z}=\left\{X_{w}: w \in \mathbf{T}\right\}$ where $X_{e^{i \theta}}=X(j)$ when $2(j-1) \pi / 3 \leq 2 j \pi / 3$ then $X_{0}=X$ and the induced centralizer $\Omega_{0}$ is equivalent to $\Omega$.

We shall not prove this in detail; the ideas are similar to those of Theorem 7.6. The key observation is that

$$
\Phi^{\Omega_{0}}=\frac{i(1-\omega)}{\pi}\left(\omega^{2} \Phi_{X(1)}+\omega \Phi_{X(2)}+\Phi_{X(3)}\right)
$$

on some strict suitable semi-ideal, where $\omega=e^{2 \pi i / 3}$. By splitting $\Phi^{\Omega}$ into its real and imaginary parts and utilizing the equation

$$
\boldsymbol{\Phi}_{X}=\frac{1}{3}\left(\boldsymbol{\Phi}_{X(1)}+\boldsymbol{\Phi}_{X(2)}+\boldsymbol{\Phi}_{X(3)}\right)
$$

one obtains equations to determine $X(j)$ for $j=1,2,3$.

\section{Applications to Schatten Classes}

We now extend our ideas to Schatten ideals (see [1] for a discussion of interpolation in this setting). Suppose $\mathscr{H}$ is a separable Hilbert space and $\mathscr{B}(\mathscr{H})$ is the algebra of all bounded operators on $\mathscr{H}$. Let $E$ be a Köthe sequence space (i.e., a Köthe function space on $\mathbf{N}$ ) which is symmetric (or rearrangement invariant) then we define the corresponding Schatten ideal $\mathscr{C}_{E}$ to be the algebra of all operators $A \in \mathscr{B}(\mathscr{H})$ whose singular values $s_{n}(A)$ satisfy $\left(s_{n}\right) \in E$. We define $\|A\|_{E}=\left\|\left(s_{n}(A)\right)\right\|_{E}$ and then $\mathscr{C}_{E}$ is a Banach space which is an ideal in $\mathscr{B}(\mathscr{H})$. Further if we regard each $A \in \mathscr{B}(\mathscr{H})$ as an infinite matrix then the spaces $\mathscr{C}_{E}$ are all admissible spaces regarded as spaces of functions on $\mathbf{N} \times \mathbf{N}$ with the usual counting measure. Thus our approach to complex interpolation derived in $\S 2$ applies to this setting.

Following [31] we define a map $\Omega: \mathscr{C}_{E} \rightarrow \mathscr{B}(\mathscr{H})$ to be a bicentralizer provided for some constant $\rho=\rho(\Omega)$ we have

$$
\|\Omega(V A W)-V \Omega(A) W\|_{E} \leq \rho\|V\|\|A\|_{E}\|W\|
$$

for $A \in \mathscr{C}_{E}$ and $V, W \in \mathscr{B}(\mathscr{H})$. By [31, Proposition 4.1] (where it is proved only for $\mathscr{C}_{p}$ ) every bicentralizer is a derivation on $\mathscr{C}_{E}$ with $\rho(\Omega) \leq 8 \rho$. Let us say that a bicentralizer is hermitian if $\Omega(H)$ is hermitian for every hermitian $H$. The argument of Lemma 7.1 yields 
Lemma 8.1. Let $\Omega$ be a bicentralizer on $\mathscr{C}_{E}$. Then there exist hermitian bicentralizers $\Omega_{1}, \Omega_{2}$ and a constant $C$ so that if $A \in \mathscr{C}_{E}$ then $\| \Omega(A)-\Omega_{1}(A)-$ $i \Omega_{2}(A)\|\leq C\| A \|_{E}$.

Let us regard $\mathscr{C}_{E}$ as a space of infinite matrices by distinguishing a particular orthonormal basis of $\mathscr{H}$. For any $A=\left(a_{j k}\right) \in \mathscr{B}(\mathscr{H})$ we define $\mathscr{D}(A)=$ $\operatorname{diag}\left(a_{j j}\right)$ and $\mathscr{D}_{n}(A)=\operatorname{diag}\left(a_{11}, \ldots, a_{n n}, 0, \ldots\right)$.

Lemma 8.2. If $\mathscr{C}_{E}$ is $B$-convex, then there is a constant $C=C(E)$ so that if $\Omega$ is a bicentralizer on $\mathscr{C}_{E}$ then for any $A \in \mathscr{C}_{E}$ we have $\|[\mathscr{D}, \Omega](A)\|_{E} \leq$ $C \rho(\Omega)\|A\|_{E}$.

Proof. By Lemma 2.1, there is a constant $C_{0}=C_{0}(E)$ so that if $A_{1}, \ldots, A_{n} \in$ $\mathscr{C}_{E}$ then

$$
\left\|\Delta_{\Omega}\left(A_{1}, \ldots, A_{n}\right)\right\|_{E} \leq C_{0} \rho \sum_{k=1}^{n}\left\|A_{k}\right\|_{E} .
$$

Hence if $U_{1}, \ldots, U_{n} \in \mathscr{B}(\mathscr{H})$ with $\left\|U_{k}\right\| \leq 1$ and if $\mathscr{T}(A)=\frac{1}{n} \sum_{k=1}^{n} U_{k} A U_{k}$ then

$$
\|[\mathscr{T}, \Omega]\|_{E}=\sup _{\|A\|_{E} \leq 1}\|[\mathscr{T}, \Omega](A)\|_{E} \leq C_{1} \rho,
$$

where $C_{1}=C_{1}(E)$.

For fixed $m \in \mathbf{N}$ let $U_{\varepsilon_{1}, \ldots, \varepsilon_{n}}=\operatorname{diag}\left(\varepsilon_{1}, \ldots, \varepsilon_{n}, 0, \ldots\right)$ where $\varepsilon_{k}= \pm 1$. By averaging over all choices of signs we obtain $\left\|\left[\mathscr{D}_{n}, \Omega\right]\right\|_{E} \leq C_{1} \rho$. Let $P_{n}$ be the orthogonal projection onto the first $n$ basis vectors. Then

$$
\left\|\Omega\left(\mathscr{D}_{n} A\right)-\mathscr{D}_{n} \Omega(A)\right\|_{E} \leq C_{1} \rho\|A\|_{E} .
$$

Thus

$$
\left\|\Omega\left(P_{n} \mathscr{D} A P_{n}\right)-\mathscr{D}_{n} \Omega(A)\right\|_{E} \leq C_{1} \rho\|A\|_{E} .
$$

Hence

$$
\left\|P_{n}(\Omega(\mathscr{D} A)-\mathscr{D} \Omega(A)) P_{n}\right\|_{E} \leq\left(C_{1}+1\right) \rho\|A\|_{E}
$$

for every $n$ and the result follows.

Theorem 8.3. Let $\mathscr{C}_{E}$ be a super-reflexive Schatten ideal. Then for every symmetric centralizer $\Omega_{E}$ on $E$ we can define a bicentralizer $\Omega$ on $\mathscr{C}_{E}$ by

$$
\Omega(A)=U^{*} \operatorname{diag}\left(\Omega_{E}\left(s_{n}\right)\right) V^{*},
$$

where $\left(s_{n}\right)$ are the singular values of $A$ and $U, V$ are norm operators chosen so that $U^{*} U=V V^{*}=I$ and $U A V=\operatorname{diag}\left(s_{n}\right)$.

Conversely, if $\Omega^{\prime}$ is a bicentralizer on $\mathscr{C}_{E}$ then there is a symmetric centralizer $\Omega_{E}$ on $E$ so that if $\Omega$ is given by (8.2) then $\Omega$ is equivalent to $\Omega^{\prime}$. Further there is a constant $\gamma=\gamma(E)>0$ so that if $\Omega^{\prime}$ is hermitian, and $\rho\left(\Omega^{\prime}\right)<\gamma$, then there are symmetric Köthe sequence spaces $E_{0}, E_{1}$ so that $\left[\mathscr{C}_{E_{0}}, \mathscr{C}_{E_{1}}\right]_{1 / 2}=\mathscr{C}_{\widetilde{E}}$ where $\widetilde{E}$ is equivalent to $E$ (i.e., has an equivalent norm), and the induced derivation is equivalent to $\Omega^{\prime}$.

Proof. We break the proof up into several steps. First consider the situation when $E_{0}, E_{1}$ are symmetric Köthe sequence spaces and $\left[E_{0}, E_{1}\right]_{1 / 2}=E$, with the induced centralizer $\Omega_{E}$. We claim that $\left[\mathscr{C}_{E_{0}}, \mathscr{C}_{E_{1}}\right]_{1 / 2}=\mathscr{C}_{E}$ and that the induced derivation the bicentralizer $\Omega$ given by (8.2). To see this suppose $\|A\|_{E}=1$ so that $\|s\|_{E}=1$. If $F(z)$ is an extremal for $A$ by considering 
$\mathscr{D}(U F(z) V)$ we must have $\|A\|_{1 / 2} \geq 1$. Conversely if $u \in B_{E_{0}}$ and $v \in B_{E_{1}}$ satisfying $s^{2}=u v$ then $F(z)=U^{*} \operatorname{diag}\left(u_{n}^{1-z} v_{n}^{z}\right) V^{*}$ is an extremal for $A$. Thus by differentiating, we may suppose that the induced derivation is given by $\Omega(A)=U^{*} \operatorname{diag}\left(\Omega_{E}(s)\right) V^{*}$, and this must be a bicentralizer by interpolation.

Now if $\mathscr{C}_{E}$ is super reflexive so that $E$ is super reflexive (and hence can be renormed to be $p$-convex and $q$-concave where $1<p<\infty$ and $\frac{1}{p}+\frac{1}{q}=1$ ) then Theorem 7.6 and Lemma 7.1 can be used to prove the first statement of the theorem.

Now suppose $\Omega^{\prime}$ is an arbitrary bicentralizer on $\mathscr{C}_{E}$. Define $\Omega_{E}$ on $E$ by $\Omega_{E}(x)=y$ where $\operatorname{diag}\left(y_{n}\right)=\mathscr{D}\left(\operatorname{diag}\left(x_{n}\right)\right)$. It is fairly easy to check that $\Omega_{E}$ is a symmetric centralizer on $E$ by considering the effect of multiplying $\operatorname{diag}\left(x_{n}\right)$ by $\operatorname{diag}\left(u_{n}\right)$ or replacing it by $S_{\pi} \operatorname{diag}\left(x_{n}\right) S_{\pi^{-1}}$ where $\pi$ is a permutation of $\mathbf{N}$ and $S_{\pi}$ is the corresponding operator obtained by permuting the basis elements. If $\|A\|_{E}=1$ and $A$ has singular values $\left(s_{n}\right)$ then we must have

$$
\left\|\Omega^{\prime}(A)-U^{*} \Omega^{\prime}\left(\operatorname{diag}\left(s_{n}\right)\right) V^{*}\right\|_{E} \leq \rho\|A\|_{E}
$$

where $\rho=\rho\left(\Omega^{\prime}\right)$. Now

$$
\left\|\Omega^{\prime}\left(\operatorname{diag}\left(s_{n}\right)\right)-\mathscr{D} \Omega^{\prime}\left(\operatorname{diag}\left(s_{n}\right)\right)\right\| \leq C \rho,
$$

where $C$ depends only on $E$. Thus

$$
\left\|\Omega^{\prime}(A)-U^{*} \operatorname{diag}\left(\Omega_{E}\left(s_{n}\right)\right) V^{*}\right\|_{E} \leq(C+1) \rho
$$

and the second part follows.

For the last statement, observe that if $\rho\left(\Omega^{\prime}\right)$ is small enough then so is $\rho\left(\Omega_{E}\right)$ and we can apply Theorem 7.6 and the first part of the proof.

We remark that the proof of Theorem 8.3 essentially classifies bicentralizers on $\mathscr{C}_{E}$ as being, up to equivalence, in bijective correspondence with symmetric centralizers on $E$. The correspondence is given by the relationship $\Omega_{0}(A)=$ $U^{*} \operatorname{diag}\left(\Omega_{0, E}\left(s_{n}\right)\right) V^{*}$ established in the proof.

Theorem 8.4. Suppose $1 \leq p_{0}<p<p_{1}<\infty$. Suppose $\mathscr{T}$ is a bounded operator on both $\mathscr{C}_{p_{0}}$ and $\mathscr{C}_{p_{1}}$. Then for every bicentralizer $\Omega$ on $\mathscr{C}_{p}$ there is a constant $C$ so that for $A \in \mathscr{C}_{p}$ we have $\|[\mathscr{T}, \Omega](A)\|_{p} \leq C\|A\|_{p}$.

The proof of Theorem 8.4 is the same as that of Corollary 7.8, using Arazy's generalization of the Boyd interpolation theorem [1] and the above Theorem 8.3. Of course, we could state Theorem 8.4 in somewhat more generality.

We conclude by studying the operator versions of indicators maps $\Phi_{X}$. Let us first notice that if $E$ is a symmetric Köthe sequence space then $l_{1} \subset E \subset l_{\infty}$ so that $\mathscr{I}_{E} \supset l \log l$. We also recall that the sequence space $h_{1}^{\text {sym }}[19,31]$ is defined to be the space of all sequences $s \in l_{1}$ such that

$$
\|s\|_{h}=\sum_{k=1}^{\infty}\left|s_{k}\right|+\sum_{k=1}^{\infty} \frac{\left|\tilde{s}_{1}+\cdots+\tilde{s}_{k}\right|}{k}<\infty,
$$

where $\tilde{s}$ is any rearrangement of $s$ so that $\left|\tilde{s}_{k}\right|$ is monotone decreasing. (Strictly, we require only that $\tilde{s}$ is a rearrangement of $s$ in the sense that if $\alpha \in \mathbf{C} \backslash\{0\}$ then the sets $\left\{k: s_{k}=\alpha\right\}$ and $\left\{k: \tilde{s}_{k}=\alpha\right\}$ have the same cardinalities; also the quasinorm \|\|$_{h}$ is well defined only up to equivalence since it depends on the choice of the rearrangement.) 
Lemma 8.5. There is a constant $C$ so that if $E$ is a symmetric Köthe sequence space, then for any $s \in h_{1}^{\mathrm{sym}} \cap l \log l$ we have $\left|\Phi_{E}(s)\right| \leq C\|s\|_{h}$.

Proof. For any sequence $\beta=\left(\beta_{n}\right)_{n=0}^{\infty}$ of complex numbers let $D(\beta)$ denote the sequence $\left(\beta_{0}, \beta_{1}, \beta_{1}, \ldots\right)$ where each $\beta_{n}$ is repeated $2^{n}$ times. Thus $\|D(\beta)\|=\sum 2^{n}\left|\beta_{n}\right|$. Next let $\beta_{n}^{\prime}=\frac{1}{2} \beta_{n-1}$ for $n \geq 1$ and $\beta_{0}^{\prime}=0$. Then it is clear that if $D(\beta) \in l \log l$ then $\left|\Phi_{E}(D(\beta))-\Phi_{E}\left(D\left(\beta^{\prime}\right)\right)\right| \leq \frac{4}{e}\|D(\beta)\|$, by Lemma 5.6. Thus if $\alpha_{0}=\beta_{0}$ and $\alpha_{n}=\beta_{n}-\frac{1}{2} \beta_{n-1}$ for $n \geq 1$ then $\left\|\Phi_{E}(D(\alpha))\right\| \leq$ $\frac{12}{e}\|D(\beta)\|$. Now if $\alpha=\left(\alpha_{n}\right)_{n=0}^{\infty}$ is any finitely nonzero sequence which satisfies $\sum^{e} 2^{n} \alpha_{n}=0$ then we can set $\beta_{0}=\alpha_{0}$ and subsequently $\beta_{n}=\sum_{k=0}^{n} 2^{k-n} \alpha_{k}$. Thus $\beta$ is finitely nonzero and we conclude that

$$
\left|\Phi_{E}(D(\alpha))\right| \leq \frac{12}{e} \sum_{n=0}^{\infty}\left|\sum_{k=1}^{n} 2^{k} \alpha_{k}\right| .
$$

Now suppose $s=\left(s_{k}\right)_{k=1}^{\infty}$ is any finitely nonzero real sequence arranged in decreasing order of absolute value. Let $\alpha_{n}=2^{-n} \sum_{k=2^{n}}^{2^{n+1}-1} s_{k}$. Also let $\theta_{n}=\left|s_{2^{n}}\right|$ for $n \geq 0$. Then we obviously have that $\sum_{n=1}^{\infty} 2^{n-1} \theta_{n} \leq\|s\|$. If $2^{n} \leq r \leq$ $2^{n+1}-1$ note that

$$
\left|\sum_{k=1}^{2^{n+1}-1} s_{k}\right| \leq\left|\sum_{k=1}^{r} s_{k}\right|+2^{n} \theta_{n}
$$

and hence for $n \geq 1$,

$$
\left|\sum_{k=0}^{n} 2^{k} \alpha_{k}\right| \leq \sum_{r=2^{n}}^{2^{n+1}-1} \frac{1}{r}\left|\sum_{k=1}^{r} s_{k}\right|+2^{n} \theta_{n} .
$$

Summing, including the obvious term when $n=0$, we obtain

$$
\left|\Phi_{E}(D(\alpha))\right| \leq 6\|s\|_{h} \text {. }
$$

Now consider $t=s-D(\alpha)$. We have $\left|t_{k}\right| \leq 2 \theta_{n}$ and $\sum_{k=2^{n}}^{2^{n+1}-1} t_{k}=0$ for $n \geq 1$ and $2^{n} \leq k \leq 2^{n+1}-1$; also $t_{1}=0$. We may thus permute the elements of $t$ in the blocks $\left(2^{n}, 2^{n}+1, \ldots, 2^{n+1}-1\right)$ to construct a sequence $\tilde{t}$ with $\left|\sum_{k=1}^{r} \tilde{t}_{k}\right| \leq 2 \theta_{n}$ for $n \geq 1$ and $2^{n} \leq r \leq 2^{n+1}-1$. Thus if $u_{r}=\sum_{k=1}^{r} \tilde{t}_{k}$ then $\|u\| \leq \sum_{n=1}^{\infty} 2^{n+1} \theta_{n} \leq 4\|s\|$. Since $\tilde{t}_{k}=u_{k}-u_{k-1}$ and $u_{0}=0$ we have $\Phi_{E}(t) \leq \frac{32}{e}\|s\|$. Combining we have that

$$
\Phi_{E}(s) \leq 22\|s\|_{h}+\frac{4}{e}(\|t\|+\|D(\alpha)\|) \leq C_{0}\|s\|_{h}
$$

for a suitable absolute constant $C_{0}$.

Next if $s$ is real but not necessarily finitely nonzero, consider for each $N$ the sequence $s^{(N)}$ defined by $s_{k}^{(N)}=s_{k}$ for $k \leq N$ and $s_{k}=0$ otherwise. Then, denoting the basis vectors by $e_{k}$,

$$
\left|\Phi_{E}\left(s^{(N)}-\sum_{k=1}^{N} s_{k} e_{N+1}\right)\right| \leq C_{0}\|s\|_{h}
$$

by the above reasoning. If $s \in h_{1}^{\text {sym }}$ then $\sum_{k=1}^{N} s_{k} \rightarrow 0$ as $N \rightarrow \infty$ and so we conclude $\lim \sup _{N \rightarrow \infty}\left|\Phi_{E}\left(s^{(N)}\right)\right| \leq C_{1}\|s\|_{h}$ for some absolute constant $C_{1}$. Let $s=s_{+}-s_{-}$be the splitting of $s$ into positive and negative parts. 
Then $\Phi_{E}\left(s_{+}\right)=\lim _{N \rightarrow \infty} \Phi_{E}\left(s_{+}^{(N)}\right)$ and $\Phi_{E}\left(s_{-}\right)=\lim _{N \rightarrow \infty} \Phi_{E}\left(s_{-}^{(N)}\right)$. It quickly follows that $\left|\Phi_{E}(s)\right| \leq C_{3}\|s\|_{h}$ for some absolute constant $C_{3}$.

Finally, if $s$ is a complex sequence the proof is completed by taking real and imaginary parts.

Let $\mathscr{C}_{l \log l}$ denote the ideal of operators in $\mathscr{C}_{1}$ whose singular value $s=\left(s_{n}\right) \in$ $l \log l$. It is easy to show that if $T \in \mathscr{C}_{l \log l}$ then the eigenvalues of $T$ repeated according to multiplicity belong to $l \log l$. (Here we adjoin zeroes if the set of eigenvalues is finite.) To see this observe that if $\left(\lambda_{n}\right)$ are the eigenvalues of $T$, arranged in decreasing order of absolute value then $\left|\lambda_{1} \cdots \lambda_{n}\right|<s_{1} \cdots s_{n}$ for every $n$ [21]. Hence since the function $x e^{x}$ is concave for $x<-2$, if we suppose, without loss of generality that $s_{1} \leq e^{-2}$ then for every $N$,

$$
\sum_{n=1}^{N}\left|\lambda_{n}\right| \log \left|\lambda_{n}\right| \geq \sum_{n=1}^{N} s_{n} \log s_{n} .
$$

Now if $E$ is a symmetric Köthe sequence space for $T \in \mathscr{C}_{l \log l}$ we define $\Phi_{E}(T)=\Phi_{E}\left(\left(\lambda_{k}\right)_{k=1}^{\infty}\right)$.

Theorem 8.6. There is a universal constant $C$ so that, whenever $E$ is a symmetric Köthe sequence space, then $\Phi_{E}$ satisfies

$$
\left|\Delta_{\Phi_{E}}\left(T_{1}, T_{2}\right)\right| \leq C\left(\left\|T_{1}\right\|_{1}+\left\|T_{2}\right\|_{1}\right)
$$

whenever $T_{1}, T_{2} \in \mathscr{C}_{l \log l}$.

Proof. Let $\lambda^{(1)}$ and $\lambda^{(2)}$ denote the eigenvalues of $T_{1}, T_{2}$ respectively both arranged in decreasing order of absolute value. Let $\nu$ denote the similar sequence for $T_{1}+T_{2}$. Then, arguing as in [19, Lemma 5.2] it may be shown that the operator $V=T_{1} \oplus T_{2} \oplus-\left(T_{1}+T_{2}\right)$ on $\mathscr{H} \oplus \mathscr{H} \oplus \mathscr{H}$ can be written as a commutator $V=[A, B]$ where $\|A\| \leq 2$ and $\|B\|_{1} \leq\left\|T_{1}\right\|_{1}+\left\|T_{2}\right\|_{1}$. Hence its eigenvalues $\alpha=\lambda^{(1)} \oplus \lambda^{(2)} \oplus(-\nu)$ are in $h_{1}^{\text {sym }}$. Further for some universal constant $C_{0}$ we have $\|\alpha\|_{h} \leq C_{0}\left(\left\|T_{1}\right\|_{1}+\left\|T_{2}\right\|_{1}\right)$. Thus $\left|\Phi_{E}(\alpha)\right| \leq C_{1}\left(\left\|T_{1}\right\|_{1}+\left\|T_{2}\right\|_{1}\right)$ for some universal $C_{1}$. It then follows quickly that $\left|\Phi_{E}(\nu)-\Phi_{E}\left(\lambda^{(1)}\right)-\Phi_{E}\left(\lambda^{(2)}\right)\right| \leq$ $C_{2}\left(\left\|T_{1}\right\|_{1}+\left\|T_{2}\right\|_{1}\right)$ from which the theorem is immediate.

\section{APPENDIX: AN APPLICATION TO HARMONIC ANALYSIS}

In this section we consider some applications to harmonic analysis. We shall suppose that $S$ is a compact metric space and that $\mu$ is a probability measure on $S$. We consider Köthe function spaces modelled on $S \times \mathbf{T}$ with the product measure $\tilde{\mu}=\mu \times \lambda$ where $d \lambda=(2 \pi)^{-1} d \theta$ is normalized Haar measure on the circle.

We will be specifically interested in the (vector-valued) Riesz transform $R$. If $x \in L_{0}(S \times \mathbf{T})$ satisfies $x_{s} \in L_{1}(\mathbf{T}) \mu$-a.e. where $x_{s}\left(e^{i t}\right)=x\left(s, e^{i t}\right)$ then we define

$$
R x\left(s, e^{i t}\right)=\lim _{r \rightarrow 1} \int_{0}^{2 \pi} \frac{x\left(s, e^{i \tau}\right)}{1-r e^{i(t-\tau)}} \frac{d \tau}{2 \pi}
$$

where the limit exists $\tilde{\mu}$-a.e.

Suppose $X$ is a Köthe function space containing $L_{\infty}$. We shall say that $R$ is bounded on $X$ if there exists a constant $C$ so that $\|R x\|_{X} \leq C\|x\|_{X}$ for all 
$x \in L_{\infty}$. If $R$ is bounded on $X$ then so are $\bar{R}$ and $R_{0}$ where $\bar{R}(f)=\overline{R(\bar{f})}$ and $R_{0}(x)=e^{i t} R\left(e^{i t} f\right)$.

If $h \in L_{1}(S)$ satisfies $h>0$ a.e. and $\int h d \mu=1$ then we say that $X$ is $h$-acceptable if $X$ contains $L_{\infty}$ and for some constant $C$ we have

$$
\int_{S \times \mathbf{T}} h(s)\left|x\left(s, e^{i t}\right)\right| d \tilde{\mu} \leq C\|x\|_{X}
$$

for all $x \in X$. If $X$ is $1_{S}$-acceptable we simply say that $X$ is acceptable. If $X$ is $h$-acceptable then $R: X \rightarrow L_{0}$ is well defined.

In this section we adopt the convention that $C$ is a constant independent of $x, y, \xi$, etc. but possibly depending on $X, X_{0}, \ldots$ which may vary from line to line.

Lemma 9.1. Suppose $X$ is a Köthe function space for which $R$ is bounded. Then there exists $h$ so that $X$ is h-acceptable and $\|R x\|_{X} \leq C\|x\|_{X}$ for all $x \in X$.

Proof. Let $J x=R x-R_{0} x$. For $x \in L_{\infty}$

$$
J x\left(s, e^{i t}\right)=\int_{0}^{2 \pi} x\left(s, e^{i \theta}\right) \frac{d \theta}{2 \pi} .
$$

Thus $\|J x\|_{X} \leq C\|x\|_{X}$ for $x \in L_{\infty}$. Let $X_{S}$ be the restriction of $X$ to $S$, so that if $x \in L_{0}(S)$ then $x \in X_{S}$ if and only if $\tilde{x} \in X$ where $\tilde{x}\left(s, e^{i t}\right)=x(s)$ and then $\|x\|_{X_{S}}=\|\tilde{x}\|_{X}$. Pick any $h \in X_{S}^{*}$ with $h>0$ a.e. and $\int h d \mu=1$. Then

$$
\int h(s)\left|x\left(s, e^{i t}\right)\right| d \tilde{\mu} \leq C\|x\|_{X}
$$

for $x \in L_{\infty}$. This clearly extends to $X$ and so $X$ is $h$-acceptable. It follows quickly that $\|R x\|_{X} \leq C\|x\|_{X}$ for all $x \in X$, since now $R$ is well defined on $X$.

If $X$ is acceptable (i.e., $L_{\infty} \subset X \subset L_{1}$ ) then $X^{*}$ is also acceptable. We shall say that $X$ is weakly acceptable if $L_{\infty} \subset X \subset L_{p}$ for some $p>0$; thus $X$ is weakly acceptable if $X^{p}$ is acceptable for some $p>0$. In this case we at least have $X^{*} \subset L_{1}$ so it follows that $L \log L \subset \mathscr{I}_{X} \cap \mathscr{I}_{X^{*}}$. It will be convenient to restrict attention largely to weakly acceptable spaces $X$ so that the indicators $\Phi_{X}$ have common domain $L \log L$. We shall also need the corresponding centralizer on $L_{1}, \Omega_{X}^{[1]}(f)=f \log |x|$ where $|f|=x x^{*}$ is the Lozanovskii factorization of $|f|$ for $X$.

Lemma 9.2. Suppose $X$ is a weakly acceptable Köthe function space on $S \times \mathbf{T}$. Then there is a constant $C$ so that if $f \in L \log L,\left\|\Omega^{[1]}(f)\right\| \leq C\|f\|_{L \log L}$.

Proof. The assumptions imply the existence of $C$ so that

$$
-C\|f\| \leq \Phi_{X}(f) \leq C\|f\|+\Lambda(f)
$$

for $f \in(L \log L)_{+}$. Thus $\left|\Phi_{X}(f)\right| \leq C\|f\|_{L \log L}$ for all $f \in L \log L$ by the quasiadditivity of $\Phi_{X}$. Hence if $\|\eta\|_{\infty} \leq 1$ and $A$ is any Borel set over which $\Omega_{X}^{[1]}(f)$ is integrable, we have

$$
\left\|\Omega^{[1]}(f) \eta 1_{A}-\Omega_{X}^{[1]}\left(f \eta 1_{A}\right)\right\| \leq C\|f\|
$$

and so

$$
\left|\int_{A} \Omega_{X}^{[1]}(f) \eta d \tilde{\mu}-\Phi_{X}\left(f \eta 1_{A}\right)\right| \leq C\|f\| .
$$


Hence $\left\|\Omega^{[1]}(f) 1_{A}\right\| \leq C\|f\|_{L \log L}$ and the lemma follows easily.

We denote by $H^{1}$ the subspace of $L_{1}(S \times \mathrm{T})$ of all $f$ such that

$$
\int_{0}^{2 \pi} f\left(s, e^{i t}\right) e^{i n t} d t=0, \quad \mu \text {-a.e. }
$$

for $n>0 . H_{0}^{1}$ is the subspace of all $f \in H^{1}$ such that

$$
\int_{0}^{2 \pi} f\left(s, e^{i t}\right) d t=0, \quad \mu \text {-a.e. }
$$

We shall need the concept of a generalized outer function. If $x \in L_{1}$ is such that for $\mu$-a.e. $s$ either $x_{s}=0 \lambda$-a.e. $\operatorname{or} \log \left|x_{s}\right| \in L_{1}(\mathbf{T})$ we define the corresponding generalized outer function $\xi \in H^{1}$ by

$$
\xi\left(s, e^{i t}\right)=\lim _{r \rightarrow 1} \exp \left(\int_{0}^{2 \pi} \frac{e^{i \tau}+r e^{i t}}{e^{i \tau}-r e^{i t}} \log \left|x\left(s, e^{i \tau}\right)\right| \frac{d \tau}{2 \pi}\right)
$$

when $\log \left|x_{s}\right| \in L_{1}$ and $\xi\left(s, e^{i t}\right)=0$ otherwise. Then $\xi$ is defined a.e. and $|\xi|=|x|$ a.e.

Proposition 9.3. Suppose $X_{0}, X_{1}$ are acceptable Köthe function spaces and that $X=\left[X_{0}, X_{1}\right]_{\theta}$ where $0<\theta<1$. Suppose that $R$ is bounded on $X$ and that $X \subset L \log L$. Let $\Omega: X \rightarrow L_{0}$ be the induced centralizer. Then $\Omega(X) \subset L_{1}$.

If, further, $R$ is bounded on $X$, and $X$ is separable, then

$$
\|[R, \Omega]\|_{X}=\sup _{\|x\|_{X} \leq 1}\|R \Omega(x)-\Omega R(x)\|_{X}<\infty
$$

if and only if there is a constant $C$ so that

$$
|\Phi(f)| \leq C\|f\|
$$

for $f \in H_{0}^{1} \cap L \log L$, where $\Phi=\Phi^{\Omega}=\Phi_{X_{1}}-\Phi_{X_{0}}$.

Proof. We first observe that if $f \in X$ then $\Omega(f)-\Omega^{[1]}(f) \in L_{1}$, where $\Omega^{[1]}$ is the corresponding centralizer on $L_{1}$, by Theorem 5.1 of [29] since $1 \in X^{*}$. However $\Omega^{[1]}=\Omega_{X_{1}}^{[1]}-\Omega_{X_{0}}^{[1]}$ and so Lemma 9.2 gives the first part of our conclusion.

We shall need the following observation. If $\xi \in L_{\infty}$ then by considering the constant function $F(z)=\xi$ for $0 \leq \mathfrak{R} z \leq 1$ we see that $(\xi, 0) \in d_{\Omega} X$ and hence $\Omega(\xi) \in X$.

Now assume $\|[R, \Omega]\|_{X}<\infty$. Suppose $f \in H_{0}^{1} \cap L \log L$ and that $\|f\|=1$. Let $|f|=x_{j} y_{j}$ be the Lozanovskii factorization of $|f|$ for $X_{j}$ for $j=0$, 1. Let $x=x_{0}^{1-\theta} x_{1}^{\theta}$ and $y=y_{0}^{1-\theta} y_{1}^{\theta}$; then $\|x\|_{X}=\|y\|_{X^{*}}=1$. Since $X$ is acceptable, $\log _{+} x, \log _{+} x^{*} \in L_{1}$. Let $S_{0}$ be the set of $s \in S$ such that $f\left(s, e^{i t}\right)$ is nonzero on a set of positive $\lambda$-measure. For $\mu$-a.e. $s \in S_{0}$ we have $f_{s} \in H^{1}(\mathbf{T})$ so that

$$
\int_{0}^{2 \pi}|\log | f_{s}\left(e^{i t}\right) \| \frac{d t}{2 \pi}<\infty
$$

and hence for almost every such $s, \log x_{s}$ is integrable. For $s \notin S_{0}, x_{s}=0$, a.e. 
Now let $\xi$ be the generalized outer function corresponding to $x$. Clearly $\xi \in B_{X} \cap H^{1}$ and $f \xi^{-1} \in B_{X^{*}} \cap H_{0}^{1}$ (here we define $f \xi^{-1}$ to be zero if $s \notin S_{0}$.) It follows from the uniqueness principle of Corollary 4.9 that

$$
\Omega(\xi)=\xi\left(\log x_{1}-\log x_{0}\right) .
$$

As $f \in L \log L$, we have $\Omega^{[1]}(f) \in L_{1}$ and hence by Theorem 5.1 of [29] we have $f \xi^{-1} \Omega(\xi) \in L_{1}$. Since $R \xi=\xi$ we have an estimate

$$
\|R \Omega(\xi)-\Omega(\xi)\|_{X} \leq C .
$$

Thus also $f \xi^{-1} R \Omega(\xi) \in L_{1}$. But $f \xi^{-1} \in H_{0}^{1}$ and $(R \Omega(\xi))_{s}$ is $\mu$-a.e. in $H_{1 / 2}$. Thus $f \xi^{-1} R \Omega(\xi) \in H_{0}^{1}$, and in particular $\int f \xi^{-1} R \Omega(\xi) d \tilde{\mu}=0$ from which it follows that $\left|\int f \xi^{-1} \Omega(\xi) d \tilde{\mu}\right| \leq C$, i.e.

$$
\left|\int f\left(\log x_{1}-\log x_{0}\right) d \tilde{\mu}\right|=|\Phi(f)| \leq C .
$$

Conversely, suppose we have the estimate $|\Phi(f)| \leq C\|f\|$ for $f \in L \log L \cap$ $H_{0}^{1}$. Suppose $\xi, \eta \in L_{\infty}$. Then both $\eta \Omega(R \xi)$ and $\eta R \Omega(\xi)$ are in $L_{1}$, and

$$
\begin{gathered}
\left|\int \eta \Omega(R \xi) d \tilde{\mu}-\Phi(\eta R \xi)\right| \leq C\|\xi\|_{X}\|\eta\|_{X^{*}}, \\
\left|\int R^{*} \eta \Omega(\xi) d \tilde{\mu}-\Phi\left(\xi R^{*} \eta\right)\right| \leq C\|\xi\|_{X}\|\eta\|_{X^{*}},
\end{gathered}
$$

by Theorem 5.1 of [29]. Now observe that $\eta R \xi-\xi R^{*} \eta=R_{0} \eta \cdot R \xi-\bar{R} \eta \cdot \bar{R}_{0} \xi$. Since $R_{0} \eta \cdot R \xi \in L \log L$ we have

$$
\left|\Phi\left(R_{0} \eta \cdot R \xi\right)\right| \leq C\left\|R_{0} \eta \cdot R \xi\right\| \leq C\|\xi\|_{X}\|\eta\|_{X^{*}} .
$$

Since $\Phi$ is real, we also have $\left|\Phi\left(\bar{R} \eta \cdot \bar{R}_{0} \xi\right)\right| \leq C\|\xi\|_{X}\|\eta\|_{X^{*}}$.

Now using the quasi-addivity of $\Phi$ this implies

$$
\left|\Phi\left(\eta R \xi-\xi R^{*} \eta\right)\right| \leq C\|\xi\|_{X} \eta \|_{X^{*}} .
$$

Hence

$$
\left|\int\left(\eta \Omega(R \xi)-R^{*} \eta \Omega(\xi)\right) d \tilde{\mu}\right| \leq C\|\dot{\xi}\|_{X}\|\eta\|_{X^{*}}
$$

and thus $\|[R, \Omega] \xi\|_{X} \leq C\|\xi\|_{X}$ for bounded $\xi$.

This inequality can now be extended to the whole of $X$, when $X$ is separable using the argument of [29, p. 82]. Notice first that if $\xi \in X$ then $R \Omega(\xi)$ is well defined. If $x \in X$ we may write $x=\sum_{n=1}^{\infty} \xi_{n}$ where $\xi_{n} \in L_{\infty}$, and $\sum_{n=1}^{\infty}\left\|\xi_{n}\right\|_{X} \leq 2\|x\|_{X}$. Let $v=\sum\left|\xi_{n}\right|$ and $w=\sum\left|R \xi_{n}\right|$. If $x_{n}=\sum_{j=1}^{n} \xi_{j}$, then

$$
\begin{gathered}
\left\|\Omega\left(x_{n}\right)-x_{n} v^{-1} \Omega(v)\right\|_{X} \leq C\|x\|_{X}, \\
\left\|R \Omega\left(x_{n}\right)-R\left(x_{n} v^{-1} \Omega(v)\right)\right\|_{X} \leq C\|x\|_{X}, \\
\left\|\Omega\left(R x_{n}\right)-\left(R x_{n}\right) w^{-1} \Omega(w)\right\|_{X} \leq C\|x\|_{X} .
\end{gathered}
$$

Combining these statements we have

$$
\left\|R\left(x_{n} v^{-1} \Omega(v)\right)-\left(R x_{n}\right) w^{-1} \Omega(w)\right\|_{X} \leq C\|x\|_{X} .
$$


As $n \rightarrow \infty$ we have $x_{n} \rightarrow x$ and $R x_{n} \rightarrow R x$ in both $X$ and $L_{0}$. By Lemma 4.3 of [29],

which implies

$$
\left\|R\left(x v^{-1} \Omega(v)\right)-(R x) w^{-1} \Omega(w)\right\|_{X} \leq C\|x\|_{x}
$$

$$
\|R \Omega(x)-\Omega(R x)\|_{X} \leq C\|x\|_{X} .
$$

We now come to the crucial calculation in our argument which essentially allows us to integrate a differential estimate for the Riesz transform.

Proposition 9.4. Given $0<\theta_{0}<1$ and $1 \leq M<\infty$ there is a constant $\delta=$ $\delta\left(\theta_{0}, M\right)>0$ so that whenever $X_{0}, X_{1}$ are weakly acceptable Köthe function spaces on $S \times \mathbf{T}$ so that $R$ is bounded on both $X_{0}$ and $X_{1}$, and such that if $X_{\theta}=\left[X_{0}, X_{1}\right]_{\theta}$ then $\|R\|_{X_{\theta_{0}}} \leq M$, and $|\Phi(f)| \leq M\|f\|$, for $f \in H_{0}^{1} \cap L \log L$, (where $\Phi=\Phi_{X_{1}}-\Phi_{X_{0}}$ ) then for $\left|\theta-\theta_{0}\right| \leq \delta$ we have $\|R\|_{X_{\theta}} \leq 2 M$.

Remark. It is important to observe that here $\delta$ is independent of the original spaces $X_{0}, X_{1}$. In the argument that follows we shall use the letter $K$ to denote a constant depending only on $\theta_{0}$ and $M$ but not on the spaces $X_{0}, X_{1}$.

Proof. Denote by $\Omega_{\theta}: X_{\theta} \rightarrow L_{0}$ the centralizer induced on $X_{\theta}, 0<\theta<1$, by the scale $\left[X_{0}, X_{1}\right]$. Similarly let $\Omega_{\theta}^{*}$ be the centralizer on $X_{\theta}^{*}$ induced by $\left[X_{0}^{*}, X_{1}^{*}\right]$. We first observe that it is possible to choose $\delta_{0}=\delta_{0}\left(\theta_{0}, M\right)>0$ and $\gamma=\gamma\left(\theta_{0}, M\right)<\infty$ (both independent of $\left.X_{0}, X_{1}\right)$ so that if $\left|\theta-\theta_{0}\right| \leq \delta_{0}$ then $\rho\left(\Omega_{\theta}\right), \rho\left(\Omega_{\theta}^{*}\right) \leq \gamma$ and we have the inequalities

$$
\begin{gathered}
\|x\|_{X_{\theta}}+\left\|y-\Omega_{\theta}\right\|_{X_{\theta}} \leq \gamma\|(x, y)\|_{d X_{\theta}}, \\
\left\|x^{*}\right\|_{X_{\theta}^{*}}+\left\|y^{*}-\Omega_{\theta}^{*} x^{*}\right\|_{X_{\theta}^{*}} \leq \gamma\left\|\left(x^{*}, y^{*}\right)\right\|_{d X_{\theta}^{*}} .
\end{gathered}
$$

Let $N(\theta), 0<\theta \leq 1$, denote the norm of $R$ on $X_{\theta}$. By our assumptions $N(\theta)$ is a bounded function and further $\log N(\theta)$ is a convex function of $\log \theta$. It follows that $N(\theta)$ is absolutely continuous on closed subintervals of $(0,1)$. Since $N(\theta) \geq 1$ for all $\theta$ the function $J(\theta)=N(\theta)^{-1}$ is similarly absolutely continuous on closed subintervals of $(0,1)$.

Now suppose that $\xi_{0}, \xi_{1}, \eta_{0}, \eta_{1}$ are bounded nonnegative functions with $\left\|\xi_{0}\right\|_{X_{0}}=\left\|\xi_{1}\right\|_{X_{1}}=\left\|\eta_{0}\right\|_{X_{0}^{*}}=\left\|\eta_{1}\right\| X_{1}^{*}=1$. Suppose also $u, v \in L_{\infty}$ with $\|u\|_{\infty}=\|v\|_{\infty} \leq 1$. Consider the function $F(z)=u \xi_{0}^{1-z} \xi_{1}^{z}$ defined in the strip $\mathscr{S}=\{z: 0<\mathfrak{R} z<1\}$. Then $F$ is analytic into $L_{\infty}$ (this can be checked by computing local Taylor series). Further if $0<\theta<1$ then $\left\|\left(F(\theta), F^{\prime}(\theta)\right)\right\|_{d X_{\theta}} \leq$ 1 and so if $\left|\theta-\theta_{0}\right| \leq \delta_{0}\left\|F^{\prime}(\theta)-\Omega_{\theta}(F(\theta))\right\|_{X_{\theta}} \leq \gamma$.

Similarly if we set $G(z)=v \eta_{0}^{1-z} \eta_{1}^{z}$, then for $\left|\theta-\theta_{0}\right| \leq \delta_{0}$,

$$
\left\|G^{\prime}(\theta)-\Omega_{\theta}^{*}(G(\theta))\right\|_{X_{\theta}^{*}} \leq \gamma .
$$

Now the map $z \mapsto R F(z)$ is analytic into $L_{2}$ and so we may define a scalarvalued analytic function $h$ on $\mathscr{S}$ by $h(z)=\int R F(z) G(z) d \tilde{\mu}$. Then

$$
h^{\prime}(\theta)=\int R^{*} G(\theta) F^{\prime}(\theta) d \tilde{\mu}+\int R F(\theta) G^{\prime}(\theta) d \tilde{\mu} .
$$

Estimating the first term we have

$$
\begin{aligned}
& \left|\int R^{*} G(\theta) F^{\prime}(\theta) d \tilde{\mu}-\int R^{*} G(\theta) \Omega_{\theta}(F(\theta)) d \tilde{\mu}\right| \\
& \leq \gamma\left\|R^{*} G(\theta)\right\|_{X_{\theta}^{*}} \leq \gamma N(\theta)\|G(\theta)\|_{X_{\theta}^{*}} \leq \gamma N(\theta) .
\end{aligned}
$$


We also have, using Theorem 5.1 of [29],

$$
\begin{array}{r}
\left\|R^{*} G(\theta) \Omega_{\theta}(F(\theta))-\Omega^{[1]}\left(R^{*} G(\theta) . F(\theta)\right)\right\| \\
\leq 36 \gamma\left\|R^{*} G(\theta)\right\|_{X_{\theta}}^{*}\|F(\theta)\|_{X_{\theta}}<K N(\theta) .
\end{array}
$$

Here $\Omega^{[1]}$ is the induced centralizer on $L_{1}$ which is independent of $\theta$, and $\Omega^{[1]}=\Omega_{X_{1}}^{[1]}-\Omega_{X_{0}}^{[1]}$. For $f \in L \log L$ we have by Lemma $8.2 \int \Omega^{[1]}(f) d \tilde{\mu}=\Phi(f)$. Thus

$$
\left|\int R^{*} G(\theta) \Omega_{\theta}(F(\theta)) d \tilde{\mu}-\Phi\left(R^{*} G(\theta) . F(\theta)\right)\right| \leq K N(\theta) .
$$

Combining the estimates we have

$$
\left|\int R^{*} G(\theta) \cdot F^{\prime}(\theta) d \tilde{\mu}-\Phi\left(R^{*} G(\theta) \cdot F(\theta)\right)\right| \leq K N(\theta) .
$$

If we let $\Phi^{*}=\Phi_{X_{1}}^{*}-\Phi_{X_{0}}^{*}$ then a precisely similar calculation shows that

$$
\left|\int R F(\theta) \cdot G^{\prime}(\theta) d \tilde{\mu}-\Phi^{*}(R F(\theta) \cdot G(\theta))\right| \leq K N(\theta) \text {. }
$$

However $\Phi^{*}=-\Phi$ and so by the quasi-additivity of $\Phi$

$$
\left|h^{\prime}(\theta)-\Phi\left(R^{*} G(\theta) \cdot F(\theta)-R F(\theta) \cdot G(\theta)\right)\right| \leq K N(\theta) .
$$

Now,

$$
R^{*} G(\theta) \cdot F(\theta)-R F(\theta) \cdot G(\theta)=\bar{R} G(\theta) \cdot \bar{R}_{0} F(\theta)-R F(\theta) \cdot R_{0} G(\theta)
$$

and so the estimate on $\Phi$ and the fact that $\Phi$ is real together give, as in the preceding proposition,

$$
\left|\Phi\left(R^{*} G(\theta) . F(\theta)-R F(\theta) \cdot G(\theta)\right)\right| \leq K N(\theta)^{2}
$$

and so $\left|h^{\prime}(\theta)\right| \leq K N(\theta)^{2}$ for $\left|\theta-\theta_{0}\right| \leq \delta_{0}$. Thus if $\theta_{0}-\delta_{0} \leq \theta_{1}<\theta_{2} \leq \theta_{0}+\delta_{0}$ we have

$$
\left|h\left(\theta_{2}\right)-h\left(\theta_{1}\right)\right| \leq K \int_{\theta_{1}}^{\theta_{2}} N(t)^{2} d t
$$

Now $\mid h(\theta) \leq N(\theta)$. Thus, it follows by taking suprema over all possible choices of $\xi_{0}, \bar{\xi}_{1}, \eta_{0}, \eta_{1}, u, v$ that

$$
\left|N\left(\theta_{2}\right)-N\left(\theta_{1}\right)\right| \leq K \int_{\theta_{1}}^{\theta_{2}} N(t)^{2} d t
$$

and at points of differentiability we obtain $\left|N^{\prime}(\theta)\right| \leq K N(\theta)^{2}$. Thus $\left|J^{\prime}(\theta)\right| \leq$ $K$ at such points from which we have the estimate $J(\theta) \geq M^{-1}-K\left|\theta-\theta_{0}\right|$ for $\left|\theta-\theta_{0}\right| \leq \delta_{0}$. The result follows.

Lemma 9.5. Suppose $p>1$ and $1 \leq M<\infty$. Then there is a constant $\delta=$ $\delta(M, p)$ with $0<\delta<1-\frac{1}{p}$ so that whenever $X$ and $Y$ are weakly acceptable Köthe function spaces, $X$ is $p$-convex and q-concave (where $\frac{1}{p}+\frac{1}{q}=1$ ), $\|R\|_{X} \leq$ $M$, and $\left|\Phi_{Y}(f)\right| \leq M\|f\|$ for $f \in H_{0}^{1} \cap L \log L$, then $\|R\|_{X Y^{a}} \leq 2 M$ for $0 \leq \alpha \leq \delta$.

Proof. We start by remarking that $X Y^{\alpha}$ is a Köthe function space for $0 \leq \alpha \leq$ $\frac{1}{q}$. 
Let $\Sigma_{N}$ be an increasing sequence of finite subalgebras of the Borel sets of $S$ whose union is $\mu$-dense. If $\Sigma_{N}$ is generated by atoms $A_{1}, \ldots, A_{m}$ we define $E_{N}$ be the conditional expectation operator of $L_{1}(S \times \mathbf{T})$ onto $L_{1}\left(\Sigma_{N}^{\prime}\right)$ where $\Sigma_{N}^{\prime}$ is generated by $A_{1} \times \mathbf{T}, \ldots, A_{m} \times \mathrm{T}$. Let $T_{N}$ be the doubly stochastic operator on $L_{1}(S \times \mathbf{T})$ defined by

$$
T_{N} f\left(s, e^{i t}\right)=\int_{0}^{2 \pi} k_{N}(t-\tau) E_{N} f\left(s, e^{i t}\right) \frac{d \tau}{2 \pi},
$$

where $k_{N}$ is the Fejer kernel

$$
k_{N}(t)=\frac{\sin ^{2}(N+1) t / 2}{(N+1) \sin ^{2} t / 2} .
$$

Now, by Theorem 5.7, there is a Köthe function space $Y_{N}$ so that $\Phi_{Y_{N}}(f)=$ $\Phi_{Y}\left(T_{N} f\right)$ for $f \in(L \log L)_{+}$. We also have that if $f \in L \log L \cap H_{0}^{1}$ then, using Corollary 5.9,

$$
\begin{aligned}
\left|\Phi_{Y_{N}}(f)\right| & \leq\left|\Phi_{Y}\left(T_{N} f\right)\right|+\frac{24}{e}\|f\| \\
& \leq\left(\frac{q M}{2}+\frac{24}{e}\right)\|f\|=K\|f\|,
\end{aligned}
$$

where $K$ is a constant depending only on $M$ and $p$, and independent of $N$. Furthermore $T_{N}$ has finite-dimensional range in $L_{\infty}$ so that for each $N$ there is a constant $B_{N}$ so that $\left|\Phi_{Y_{N}}(f)\right| \leq B_{N}\|f\|$ for all $f \in L \log L$.

From the convexity and concavity conditions on $X$ it follows that we may define Köthe function spaces $Z_{0}^{N}, Z_{1}^{N}$ by the equations $\Phi_{Z_{1}^{N}}=\Phi_{X}+\frac{1}{q} \Phi_{Y_{N}}$ and $\Phi_{Z_{0}^{N}}=\Phi_{X}-\frac{1}{q} \Phi_{Y_{N}}$. Then each $Z_{0}^{N}, Z_{1}^{N}$ is simply a renorming of $X$ so that $R$ is bounded on both spaces. Furthermore if $f \in L \log L \cap H_{0}^{1}$,

$$
\left|\Phi_{Z_{1}^{N}}(f)-\Phi_{Z_{0}^{N}}(f)\right|=\frac{2}{q}\left|\Phi_{Y_{N}}(f)\right| \leq K\|f\|,
$$

where $K$ again depends only on $M$ and $p$. We are thus in a position to apply Lemma 8.4. There exists $0<\delta_{0}<\frac{1}{2}$ depending only on $M$ and $p$, so that if $\left|\theta-\frac{1}{2}\right| \leq \delta_{0}$ then $\|R\|_{Z_{\theta}} \leq 2 M$, where $Z_{\theta}=\left[Z_{0}, Z_{1}\right]_{\theta}$.

Now suppose $\delta=\frac{2}{q} \delta_{0}$ and that $0 \leq \alpha \leq \delta$. To prove the lemma, it suffices to consider $\xi \in X Y^{\alpha} \cap L_{\infty}$ of the form $\xi=u|x||y|^{\alpha}$ where $u, x, y \in L_{\infty}$, $\|u\|_{\infty},\|x\|_{X},\|y\|_{X} \leq 1$, and we additionally suppose that $\log |y| \in L_{\infty}$.

Let $y_{N}=\exp \left(T_{N} \log |y|\right)$. For any $f \in(L \log L)_{+}$we have

$$
\int f \log y_{N} d \tilde{\mu}=\int T_{N} f \log |y| d \tilde{\mu} \leq \Phi_{Y}\left(T_{N} f\right)=\Phi_{Y_{N}}(f)
$$

so that $y_{N} \in B_{Y_{N}}$. If we let $\xi_{N}=u|x| y_{N}^{\alpha}$ then $\left\|\xi_{N}\right\|_{Z_{\theta}^{N}} \leq 1$, where $\theta-\frac{1}{2}=$ $\frac{q}{2} \alpha \leq \delta_{0}$. Hence $\left\|R \xi_{N}\right\|_{Z_{\theta}^{N}} \leq 2 M$.

Now, as $N \rightarrow \infty$ we have $\left\|R \xi_{N}-R \xi\right\|_{2} \rightarrow 0$. Let $A$ be the measurable subset of $S \times \mathbf{T}$ where $\log |R \xi|>-\infty$. For any $\eta>0$ we may find a subset $A_{\eta}$ of $A$ with $\tilde{\mu}\left(A \backslash A_{\eta}\right)<\eta$ and a subsequence such that $\log \left|R \xi_{N_{l}}\right|$ converges 
uniformly on $A_{\eta}$ to $\log |R \eta|$. Now suppose $f \in\left(L_{\infty}\right)_{+}$.

$$
\begin{aligned}
\int_{A_{\eta}} f \log |R \eta| d \tilde{\mu} & =\lim _{l \rightarrow \infty} \int_{A_{\eta}} f \log \left|R \xi_{N_{l}}\right| d \tilde{\mu} \\
& \leq \limsup _{l \rightarrow \infty}\left(\log (2 M)+\Phi_{Z_{\theta}^{N_{l}}}\left(f 1_{A_{\eta}}\right)\right) \\
& \leq \log (2 M)+\underset{l \rightarrow \infty}{\lim \sup }\left(\Phi_{X}\left(f 1_{A_{\eta}}\right)+\alpha \Phi_{Y}\left(T_{N_{l}}\left(f 1_{A_{\eta}}\right)\right)\right. \\
& =\log (2 M)+\Phi_{X}\left(f 1_{A_{\eta}}\right)+\alpha \Phi_{Y}\left(f 1_{A_{\eta}}\right)
\end{aligned}
$$

where the last step uses the continuity of $\Phi_{Y}$ on order intervals. Using this same continuity we obtain

$$
\int_{A} f \log |R \xi| d \tilde{\mu} \leq \log (2 M)+\Phi_{X}\left(f 1_{A}\right)+\alpha \Phi_{Y}\left(f 1_{A}\right) .
$$

Now it follows that we have

$$
\int f \log |R \xi| d \tilde{\mu} \leq \log (2 M)+\Phi_{X}(f)+\alpha \Phi_{Y}(f),
$$

since if $f-f 1_{A} \neq 0$ then the integral is $-\infty$. Hence $\|R \xi\|_{X Y^{\alpha}} \leq 2 M$ as required.

Lemma 9.6. Suppose $X$ is an acceptable Köthe function space and that $R$ is bounded on $X$. Then there is a constant $M$ so that $\left|\Phi_{X}(f)\right| \leq M\|f\|$ for $f \in H_{0}^{1} \cap L \log L$.

Proof. Clearly $R$ is bounded on both $X$ and $X^{*}$. By interpolation we obtain that $[R, \Omega]$ is bounded on $L_{2}=\left[X, X^{*}\right]_{1 / 2}$ where $\Omega$ is the induced centralizer. By Proposition 8.3 there exists $B$ so that

$$
\left|\Phi_{X}(f)-\Phi_{X^{*}}(f)\right|=\left|2 \Phi_{X}(f)-\Lambda(f)\right| \leq B\|f\|
$$

for $f \in H_{0}^{1} \cap L \log L$. Applying the same reasoning to $X=L_{p}$ where $1<p<2$ shows that $|\Lambda(f)| \leq B^{\prime}\|f\|$ for $f \in H_{0}^{1} \cap L \log L$. The lemma follows.

We now state our main theorem of the section.

Theorem 9.7. Let $X_{0}, X_{1}$ be acceptable Köthe function spaces on $S \times \mathbf{T}$. Suppose $0<\theta_{0}<1$ and that $X=\left[X_{0}, X_{1}\right]_{\theta_{0}}$ is super reflexive and satisfies $L_{r} \subset X \subset L_{s}$ for some $1<s<r<\infty$. Suppose also that $R$ is bounded on $X$. Let $\Omega$ be the induced centralizer on $X$. Then the following conditions are equivalent.

(1) $\|[R, \Omega]\|_{X}<\infty$.

(2) There exists $\delta>0$ so that $R$ is bounded on $X_{\theta}$ for $\left|\theta-\theta_{0}\right| \leq \delta$.

(3) For some constant $M$ we have $\left|\Phi_{X_{j}}(f)\right| \leq M\|f\|$ for $j=0,1$ whenever $f \in H_{0}^{1} \cap L \log L$.

Proof. $(1) \Rightarrow(3)$. This follows by combining Proposition 9.3 which yields an estimate for $\Phi_{X_{1}}-\Phi_{X_{0}}$ and Lemma 9.6 which yields an estimate for $\theta_{0} \Phi_{X_{1}}+$ $\left(1-\theta_{0}\right) \Phi_{X_{0}}$.

(3) $\Rightarrow(2)$. Let $Y$ be a renorming of $X$ which is $p$-convex and $q$-concave where $p>1$ and $\frac{1}{p}+\frac{1}{q}=1$. Assume $\|R\|_{Y}=M$. Then (by Lemma 9.5) there exists $\delta_{0}=\delta_{0}(r, p, M)>0$ so that if $0 \leq \alpha \leq \delta_{0}$ then $Y X_{1}^{\alpha}$ is acceptable, $\|R\|_{Y X_{1}^{\alpha}} \leq 2 M$, and $Y X_{1}^{\alpha}$ is both $2 q$-concave and $p^{\prime}$-convex where $\frac{1}{p^{\prime}}+\frac{1}{2 q}=1$. 
Using Lemma 9.5 on $\left(Y X_{1}^{\alpha}\right)^{*}$ there exists $\delta_{1}=\delta_{1}(r, p, M)>0$ so that if $0 \leq \beta \leq \delta_{1}$ then $\|R\|_{Z} \leq 4 M$ where $Z=\left(Y X_{1}^{\alpha}\right)^{*}\left(X_{0}\right)^{\beta}$. In particular if $\alpha \leq \delta=\min \left(\delta_{0}, \delta_{1}\right)$ then $R$ is bounded on $Z_{\alpha}=\left(Y X_{1}^{\alpha}\right)^{*}\left(X_{0}\right)^{\alpha}$. Now

$$
\begin{aligned}
\boldsymbol{\Phi}_{Z_{\alpha}} & =\boldsymbol{\Phi}_{\left(Y X_{1}^{\alpha}\right)}^{*}+\alpha \boldsymbol{\Phi}_{X_{0}}=\Lambda-\left(\boldsymbol{\Phi}_{Y}+\alpha \boldsymbol{\Phi}_{X_{1}}\right)+\alpha \boldsymbol{\Phi}_{X_{0}} \\
& =\Lambda-\left(\boldsymbol{\Phi}_{Y}+\alpha\left(\boldsymbol{\Phi}_{X_{1}}-\boldsymbol{\Phi}_{X_{0}}\right)\right)
\end{aligned}
$$

and hence $Z_{\alpha}^{*}$ is a renorming of $X_{\theta_{0}+\alpha}$ whence $R$ is bounded on this space for $0 \leq \alpha \leq \delta$. A similar argument gives the case $-\delta \leq \alpha \leq 0$.

$(2) \Rightarrow(1)$. This is simply Theorem 2.7 .

Let us now specialize to the case when $S$ reduces to one point so that $S \times \mathbf{T}$ is simply $\mathbf{T}$. In this case $R$ cannot be bounded unless $X$ is acceptable. Then it follows from Lemma 9.5 that $X L_{1}^{\alpha}$ is also acceptable for some $\alpha>0$ so that $X \subset L_{s}$ for some $s>1$. By duality $X^{*} \supset L_{r}$ for some finite $r$. It then follows that in the hypotheses of Theorem 9.7 we need only assume $X_{0}, X_{1}$ weakly acceptable, since then for some $\alpha<\theta_{0}<\beta$ we will have $X_{\alpha}, X_{\beta}$ acceptable. Thus we restate our result, adding one more equivalence.

Theorem 9.8. Let $X_{0}, X_{1}$ be weakly acceptable Köthe function spaces on $\mathbf{T}$. Suppose $0<\theta_{0}<1$ and that $X=\left[X_{0}, X_{1}\right]_{\theta_{0}}$ is super reflexive and $R$ is bounded on $X$. Then the following are equivalent.

(1) $\|[R, \Omega]\|_{X}<\infty$.

(2) There exists $\delta>0$ so that $R$ is bounded on $X_{\theta}$ for $\left|\theta-\theta_{0}\right|<\delta$.

(3) For some constant $C$ we have $\left|\Phi_{X_{j}}(f)\right| \leq C\|f\|$ for $f \in H_{0}^{1} \cap L \log L$ and $j=0,1$.

(4) The set $\left\{\theta:\|R\|_{X_{\theta}}<\infty\right\}$ is open.

Let us now illustrate this result. Suppose $w$ is an $A_{p}$-weight where $1<$ $p<\infty$, i.e. $R$ is bounded on $L_{p}(w)$. Then $R$ is bounded on $L_{p}\left(w^{\theta}\right)$ for $0 \leq \theta \leq 1$. An application of (3) of Theorem 9.8 gives

$$
\left|\int_{\mathbf{T}} f \log w d \lambda\right| \leq C\|f\|
$$

for $f \in H_{0}^{1} \cap L \log L$. The point is that if $X=L_{p}(w)$ then $\Phi_{X}(f)=\frac{1}{p} \Lambda(f)-$ $\int f \log w d \lambda$. Thus $\log w \in$ BMO.

Conversely if $\log w \in$ BMO then taking $X_{0}=L_{p}\left(w^{-1}\right)$ and $X_{1}=L_{p}(w)$ we see that $R$ is bounded on $L_{p}\left(w^{\alpha}\right)$ for some $\alpha>0$, i.e. $w^{\alpha}$ is an $A_{p}$-weight. Thus we have the following result of Coifman-Rochberg [13] as a special case.

Corollary 9.9. Suppose $1<p<\infty$. Then $u \in \operatorname{BMO}(\mathbf{T})$ if and only if $e^{\alpha u}$ is an $A_{p}$-weight for some $\alpha>0$.

Further well-known facts can also be recovered this way. If $1<p<2$ and $w$ is an $A_{p}$-weight, let $X_{0}=L_{2}$, and $X_{1}=L_{1}\left(w^{\alpha}\right)$ where $\alpha^{-1}=2-p$. Then $L_{p}(w)=X_{\theta}$ where $\theta=\alpha^{-1}$. Since $R$ is bounded at both $X_{0}$ and $X_{\theta}$ it is clear that (3) and hence (4) of Theorem 9.8 hold. Thus $R$ is bounded on $L_{p-\varepsilon}\left(w^{\beta}\right)$ for some $\varepsilon>0$ and $\beta>1$. By further interpolation $w$ is an $A_{p-\varepsilon}$-weight. (See Muckenhoupt [41].)

Let us now turn to the vector valued case. Suppose $X$ is a super reflexive Köthe function space on $S$. Then $X$ is a (UMD)-space (Burkholder [7]) if 
and only if $R$ is bounded on the space $L_{2}(X)$ ([4, 8]) where $L_{2}(X)$ may be identified with the Köthe function space on $S \times \mathrm{T}$ of all $x$ such that

$$
\|x\|_{L_{2}(X)}=\left(\int_{0}^{2 \pi}\left\|x_{t}\right\|_{X}^{2} \frac{d t}{2 \pi}\right)<\infty,
$$

where $x_{t}(s)=x\left(s, e^{i t}\right)$. We now state our main theorem.

Theorem 9.10. Let $X_{0}, X_{1}$ be Köthe function spaces on $S$ and that $X=$ $\left[X_{0}, X_{1}\right]_{\theta_{0}}$ is a (UMD)-space. Let $\Omega$ be the induced centralizer at $X$. Then $d_{\Omega} X$ is a (UMD)-space if and only if there exists $\delta>0$ so that $X_{\theta}$ is a (UMD)space for $\left|\theta-\theta_{0}\right| \leq \delta$.

Proof. Let us first prove the assertion under the additional hypotheses that $X_{0}, X_{1}$ are acceptable and $L_{r} \subset X \subset L_{s}$ where $1<s<r<\infty$. Under these hypotheses, Theorem 9.7 applies to the interpolation scale

$$
L_{2}\left(X_{\theta}\right)=\left[L_{2}\left(X_{0}\right), L_{2}\left(X_{1}\right)\right]_{\theta} \text {. }
$$

The proof will be complete when we show that $d_{\Omega} X$ is (UMD) if and only if the induced centralizer $\widetilde{\Omega}$ at $L_{2}(X)$ satisfies $[R, \widetilde{\Omega}]_{L_{2}(X)}<\infty$. It will be convenient to suppose $X_{0}, X_{1}$ are renormed to be strictly convex Köthe function spaces; this involves no loss of generality, and results in uniqueness of the choice of extremal (see Theorem 2.6 and remarks).

If $x \in L_{2}(X)$ and $\|x\|_{L_{2}(X)}=1$ then we write $x=u|x|$ where $|u|=1$ a.e. Let $|x|=\left|x_{0}\right|^{1-\theta_{0}}\left|x_{1}\right|^{\theta_{0}}$ where $\left\|x_{0}\right\|_{L_{2}\left(X_{0}\right)}=\left\|x_{1}\right\|_{L_{2}\left(X_{1}\right)}=1$. By Hölder's inequality $\left\|x_{t}\right\|_{X}=\left\|x_{0, t}\right\|_{X_{0}}=\left\|x_{1, t}\right\|_{X_{1}}=1$ a.e. Thus, utilizing the uniqueness of both $\Omega$ and $\widetilde{\Omega}$

$$
\begin{aligned}
\widetilde{\Omega}(x) & =x\left(\log \left|x_{1}\right|-\log \left|x_{0}\right|\right), \\
(\widetilde{\Omega}(x))_{t} & =x_{t}\left(\log \left|x_{1, t}\right|-\log \left|x_{0, t}\right|\right)=(\Omega(x))_{t} \quad \text { a.e. }
\end{aligned}
$$

Now if $\phi, \psi \in L_{0}(S \times \mathbf{T})$, then $\|(\phi, \psi)\|_{L_{2}\left(d_{\Omega} X\right)}$ is equivalent to

$$
\left(\int\left\|\phi_{t}\right\|_{X}^{2} d \lambda\right)^{1 / 2}+\left(\int\left\|\psi_{t}-\Omega\left(\phi_{t}\right)\right\|_{X}^{2} d \lambda\right)^{1 / 2}
$$

Here to avoid cumbersome measurability problems we may interpret the integrals as upper integrals; however, it can be verified that all functions are suitably measurable. Thus $\|(\phi, \psi)\|_{d_{\Omega} X}$ is equivalent to $\|\phi\|_{L_{2}(X)}+\|\psi-\widetilde{\Omega}(\psi)\|_{L_{2}(X)}$ and $L_{2}\left(d_{\Omega} X\right)$ can be identified with $d_{\widetilde{\Omega}}\left(L_{2}(X)\right)$. Thus the vector-valued Riesz transform $R$ is bounded on $L_{2}\left(d_{\Omega} X\right)$ if and only if the map $(\phi, \psi) \mapsto(R \phi, R \psi)$ is bounded on $d_{\widetilde{\Omega}}\left(L_{2}(X)\right)$ i.e., if and only if $\|[R, \widetilde{\Omega}]\|_{L_{2}(X)}<\infty$. The result will then reduce to Theorem 9.7.

It remains to justify our hypotheses. Since $X$ is super reflexive it is equivalent to a space which is $p$-convex and $q$-concave where $\frac{1}{p}+\frac{1}{q}=1$ and $p>1$. By Pisier's theorem [43], $X$ is equivalent to $\left[L_{2}, Y\right]_{\tau}$ where $Y$ is a Köthe function space and $\tau>0$. Now, from Lozanovksii's theorem, there is a weight function $w_{2}>0$ a.e. such that $L_{\infty} \subset w_{2} Y \subset L_{1}$. Here $\|x\|_{w Y}=\left\|x w^{-1}\right\|_{Y}$. Similarly there exist $w_{0}, w_{1}$ so that $L_{\infty} \subset w_{0} X_{0}, w_{1} X_{1} \subset L_{1}$. Now $L_{2 /(1-\tau)} \subset$ $w_{2}^{\tau} X \subset L_{2 /(1+\tau)}$, so that for some $\eta>0$ we can find weights, $w_{3}, w_{4}$ with 
$L_{\alpha} \subset w_{3} X_{\theta_{0_{-\eta}}}, w_{4} X_{\theta_{0}+\eta} \subset L_{\beta}$ with $1<\beta<\alpha<\infty$. Thus the problem may be reduced by a change of weight to the type discussed above.

Theorem 9.11. There exists a centralizer $\Omega$ on $L_{2}(0,1)$ so that the twisted sum $d_{\Omega} L_{2}$ is not (UMD). Thus the (UMD)-property is not a three-space property.

Proof. Bourgain [4] shows that for any $p<2$ there is a Köthe function space $X_{p}$ on $(0,1)$ which is $p$-convex and $q$-concave but not (UMD). By Pisier's theorem [43] if $0<\theta<1$ there exists a Köthe space $X^{[\theta]}$ so that $\left[L_{2}, X^{[\theta]}\right]_{\theta}$ fails to be (UMD). It follows by combining isomorphic copies of the spaces $X^{[1 / n]}$ as bands in a single space $X$ that there is a Köthe function space $X$ so that $\left[X^{*}, X\right]_{\theta}$ is (UMD) if and only if $\theta=\frac{1}{2}$ when $X_{\theta}=L_{2}$. If $\Omega$ is the induced centralizer, $d_{\Omega} L_{2}$ cannot be (UMD).

Theorem 9.12. If $X_{0}, X_{1}$ be Köthe function spaces on $S$. Then the set $\{\theta: 0<$ $\theta<1$, and $X_{\theta}$ is (UMD) $\}$ is either open or a single point.

We omit the proof of Theorem 9.12 which is similar to Theorem 9.8; this theorem extends a result of Rubio de Francia [46].

Added in proof. See also [51] where the similar results to Theorem 9.8 and 9.12 are conjectured.

\section{REFERENCES}

1. J. Arazy, Some remarks on interpolation theorems and the boundedness of the triangular projection in unitary matrix spaces, Integral Equations Operator Theory 1 (1978), 453-495.

2. C. Bennett and R. Sharpley, Interpolation of operators, Academic Press, New York, 1988.

3. J. Bergh and J. Lofstrom, Interpolation spaces: an introduction, Springer-Verlag, Berlin, 1976.

4. J. Bourgain, Some remarks on Banach spaces in which martingale difference sequences are unconditional, Ark. Mat. 21 (1983), 163-168.

5. D. W. Boyd, Indices of function spaces and their relationship to interpolation, Canad. J. Math. 21 (1969), 1245-1254.

6. A. V. Bukvhalov and A. A. Danilevich, Boundary properties of analytic functions with values in a Banach space, Mat. Zametki 31 (1982) 103-114 = Math. Notes 31 (1982), 104-110.

7. D. L. Burkholder, A geometrical characterization of Banach spaces in which martingale difference sequences are unconditional, Ann. Probab. 9 (1981), 997-1011.

8. __ A geometric condition that implies the existence of certain singular integrals of Banach space-valued functions, Conference on Harmonic Analysis in Honor of A. Zygmund (W. Beckner, A. P. Calderon, R. Fefferman, P. W. Jones, eds.), Wadsworth, Belmont, Calif., 1983, pp. 270-286.

9. A. P. Calderon, Intermediate spaces and interpolation, the complex method, Studia Math. 24 (1964), 113-190.

10. O. D. Ceretelli, A metric characterization of the set of functions whose conjugate functions are integrable, Bull. Acad. Sci. Georgian SSR 81 (1976), 281-283. (Russian)

11. R. R. Coifman, M. Cwikel, R. Rochberg, Y. Saghar, and G. Weiss, The complex method for interpolation of operators acting on families of Banach spaces, Euclidean Harmonic Analysis (J. Benedetto, ed.), Lecture Notes in Math., vol. 779, Springer-Verlag, Berlin and New York, 1980.

12. _ A theory of complex interpolation for families of Banach spaces, Adv. Math. 33 (1982), 203-229.

13. R. R. Coifman and R. Rochberg, Another characterization of BMO, Proc. Amer. Math. Soc. 79 (1980), 249-254. 
14. R. R. Coifman and S. Semmes Interpolation of Banach spaces, Perron processes, and YangMills (to appear).

15. M. Cwikel, B. Jawerth, M. Milman, and R. Rochberg, Differential estimates and commutators in interpolation theory, Analysis at Urbana II (E. R. Berkson, N. T. Peck, and J. Uhl, eds.), London Math. Soc. Lecture Notes Ser., vol. 138, Cambridge Univ. Press, Cambridge, 1989, pp. 170-220.

16. M. Cwikel and P. Nilsson, Interpolation of weighted Banach lattices (to appear).

17. B. Davis, Hardy spaces and rearrangements, Trans. Amer. Math. Soc. 261 (1980), 211-233.

18. P. L. Duren, Theory of $H^{p}$-spaces, Academic Press, New York, 1970.

19. C. M. Eoff and N. J. Kalton, Zeros of entire functions and characteristic determinants, Complex Variables 14 (1990), 53-65.

20. T. A. Gillespie, Factorization in Banach function spaces, Indag. Math. 43 (1981), 287-300.

21. I. C. Gohberg and M. G. Krein, Introduction to the theory of linear nonselfadjoint operators, Translations Math. Mono., vol. 18, Amer. Math. Soc., Providence, R.I., 1969.

22. E. Hernandez, Intermediate spaces and the complex method of interpolation for families of Banach spaces, Ann. Scuola Norm. Sup. Pisa Cl. Sci. 13 (1986), 245-266.

23. B. Jawerth, R. Rochberg, and G. Weiss, Commutator and other second order estimates in real interpolation theory, Ark. Mat. 24 (1986), 191-210.

24. N. J. Kalton, The three space problem for locally bounded F-spaces, Compositio Math. 37 (1978), 243-276.

25. __ Convexity, type, and the three-space problem, Studia Math. 69 (1980), 57-96.

26. _ _ The space $Z_{2}$ viewed as a symplectic Banach space, Proc. Res. Workshop on Banach Space Theory, Univ. of Iowa, 1981, pp. 97-111.

27. __ Applications of an inequality for $H_{1}$-functions, Longhorn Notes, Functional Analysis Seminar, Univ. of Texas at Austin, 1985/6.

28. __ Plurisubharmonic functions on quasi-Banach spaces, Studia Math. 84 (1986), 297324.

29. __ Nonlinear commutators in interpolation theory, Mem. Amer. Math. Soc. No. 385, 1988.

30. _ _ On rearrangements of vector-valued $H_{1}$-functions, Ark. Mat. 26 (1988), 221-229.

31. _ Trace-class operators and commutators, J. Funct. Anal. 86 (1989), 41-74.

32. N. J. Kalton and N. T. Peck, Twisted sums of sequence spaces and the three space problem, Trans. Amer. Math. Soc. 255 (1979), 1-30.

33. N. J. Kalton, N. T. Peck, and J. W. Roberts, An F-space sampler, London Math. Soc. Lecture Notes Ser., vol. 89, Cambridge Univ. Press, 1985.

34. N. J. Kalton and J. W. Roberts, Uniformly exhaustive submeasures and nearly additive set functions, Trans. Amer. Math. Soc. 278 (1983), 803-816.

35. N. J. Kalton and R. C. Swanson, A symplectic Banach space with no Lagrangian subspaces, Trans. Amer. Math. Soc. 273 (1982), 385-392.

36. J. Komlos, A generalization of a problem of Steinhaus, Acta Math. Acad. Sci. Hungar. 18 (1967), 217-229.

37. J. Lindenstrauss and L. Tzafriri, Classical Banach spaces. II, Function spaces, Ergeb. Math. Grenzgeb., vol. 97, Springer-Verlag, Berlin, Heidelberg, and New York, 1979.

38. G. Y. Lozanovskii, On some Banach lattices, Siberian Math. J. 10 (1969), 419-430.

39. B. Maurey, Nouveaux théorèmes de Nikishin, Exposeés 4-5, Sem. Maurey-Schwartz, 19741974.

40. M. Milman and T. Schonbek, $A$ note on second order estimates in interpolation theory and applications, Proc. Amer. Math. Soc. 110 (1990), 961-969.

41. B. Muckenhoupt, Weighted norm inequalities for the Hardy maximal function, Trans. Amer. Math. Soc. 165 (1972), 207-226.

42. E. M. Nikishin, Resonance theorems and superlinear operators, Uspekhi Mat. Nauk 25 (1970), 129-191 = Russian Math. Surveys 25 (1970), 124-187. 
43. G. Pisier, Some applications of the complex interpolation method to Banach lattices, J. Analyse Math. 35 (1979), 264-281.

44. M. Ribe, Examples for the non-locally convex three space problem, Proc. Amer. Math. Soc. 73 (1979), 351-355.

45. R. Rochberg and G. Weiss, Derivatives of analytic families of Banach spaces, Ann. of Math. 118 (1983), 315-347.

46. J. L. Rubio de Francia, Martingale and integral transforms of Banach space valued functions, Probability and Banach spaces, Proceedings, Zaragoza, 1985 (J. Bastero and M. San Miguel, eds.), Lecture Notes in Math., vol. 1221, Springer-Verlag, Berlin and New York, 1986, pp. 195-222.

47. M. Schechter, Complex interpolation, Compositio Math. 18 (1967), 117-147.

48. S. Semmes, Interpolation of Banach spaces, differential geometry, and differential equations, Rev. Mat. Iberoamericana 4 (1988), 155-176.

49. Z. Slodkowski, Complex interpolation of normed and quasinormed spaces in several dimensions. I, Trans. Amer. Math. Soc. 308 (1988), 685-711.

50. A. Torchinsky, Real-variable methods in harmonic analysis, Academic Press, New York, 1986.

51. J. L. Rubio de Francia, Operators in Banach lattices and weighted $L^{2}$ inequalities, Math. Nachr. 133 (1987), 197-209.

Department of Mathematics, University of Missouri, Columbia, Missouri 65211

E-mail address: mathnjk@mizzoul.bitnet 